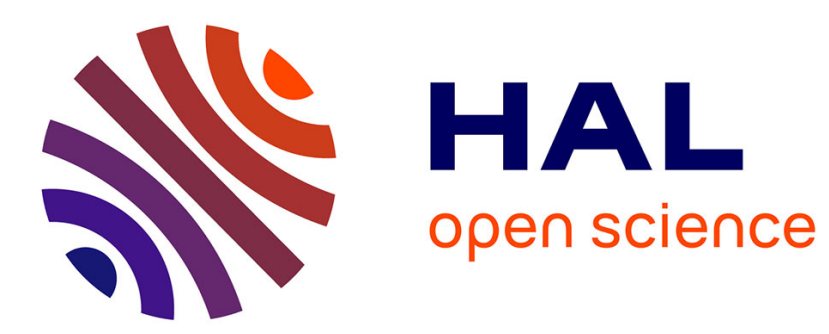

\title{
Progress of in situ synchrotron X-ray diffraction studies on the mechanical behavior of materials at small scales
}

Thomas W. Cornelius, Olivier Thomas

\section{To cite this version:}

Thomas W. Cornelius, Olivier Thomas. Progress of in situ synchrotron X-ray diffraction studies on the mechanical behavior of materials at small scales. Progress in Materials Science, 2018, 94, pp.384-434. 10.1016/j.pmatsci.2018.01.004 . hal-01793295

\section{HAL Id: hal-01793295 \\ https://hal.science/hal-01793295}

Submitted on 28 May 2018

HAL is a multi-disciplinary open access archive for the deposit and dissemination of scientific research documents, whether they are published or not. The documents may come from teaching and research institutions in France or abroad, or from public or private research centers.
L'archive ouverte pluridisciplinaire HAL, est destinée au dépôt et à la diffusion de documents scientifiques de niveau recherche, publiés ou non, émanant des établissements d'enseignement et de recherche français ou étrangers, des laboratoires publics ou privés. 


\section{Progress of in situ synchrotron X-ray diffraction studies on the mechanical behavior of materials at small scales}

Thomas W. Cornelius and Olivier Thomas

Aix Marseille Univ, Univ Toulon, CNRS, IM2NP, Marseille, France

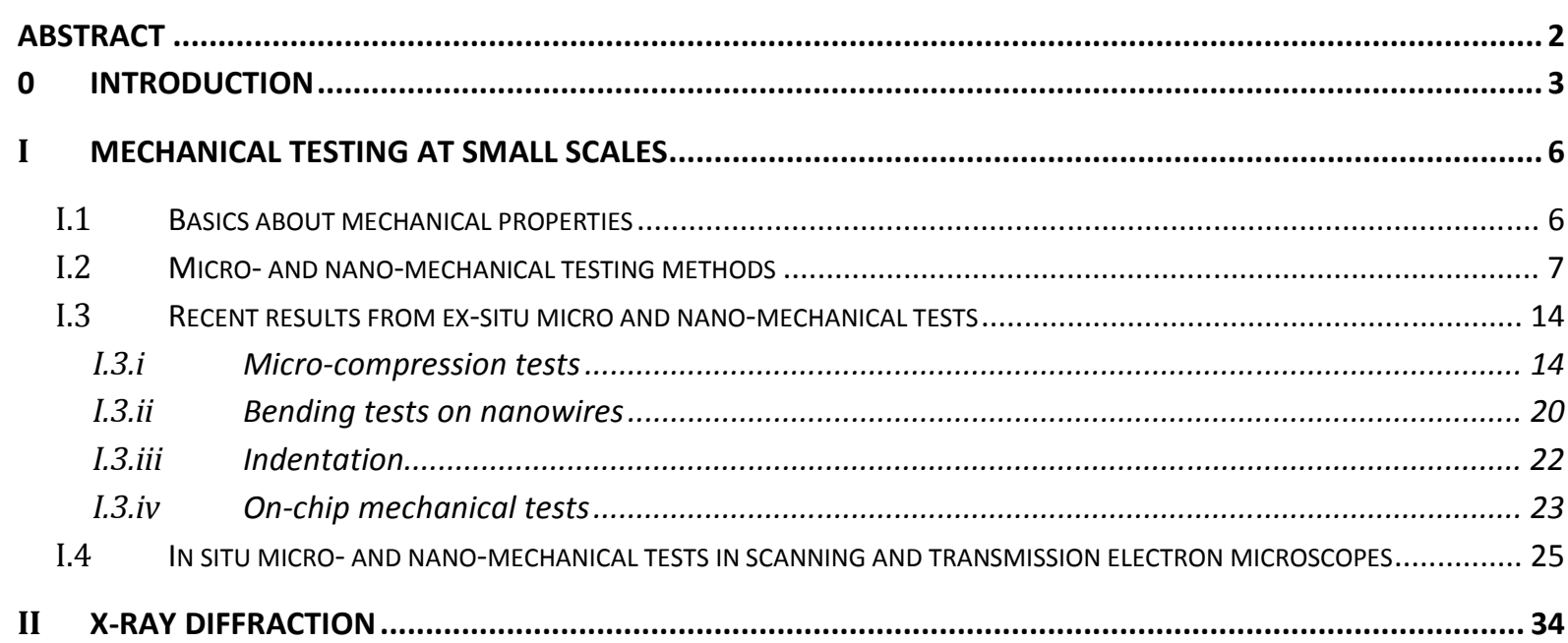

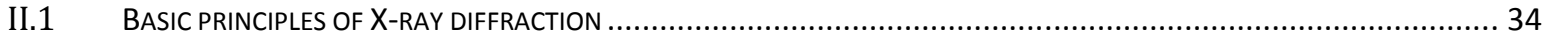

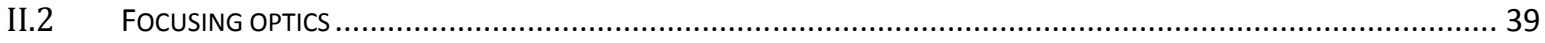

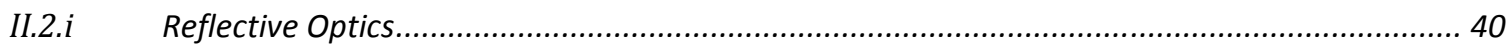

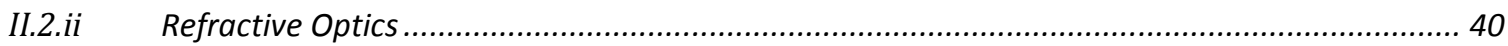

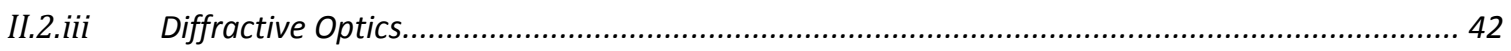

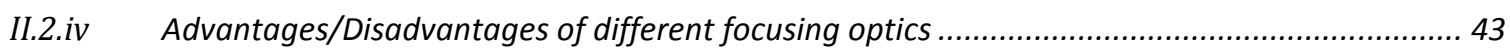

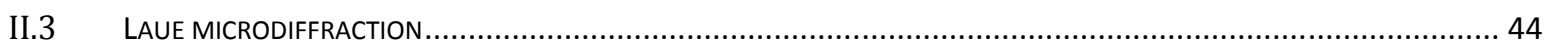

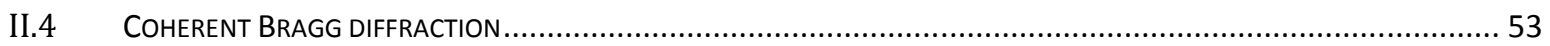

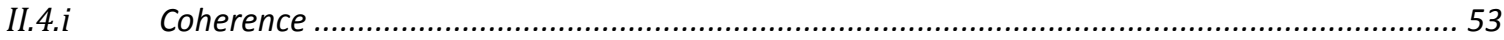

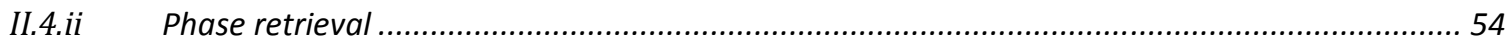

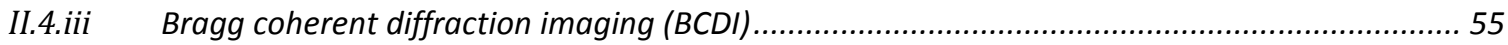

III PROGRESS OF IN SITU MICRO- AND NANO-MECHANICAL TESTING IN COMBINATION WITH

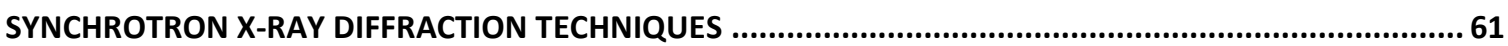

III.1 IN SITU MICRO- AND NANO-MECHANICAL TESTS IN COMBINATION WITH LAUE MICRODIFFRACTION...........................63

III.1.i In situ micro-compression tests combined with Laue microdiffraction.......................................6.6. 64

III.1.ii In situ nanowire bending tests combined with Laue microdiffraction .......................................67

III.2 IN SITU MICRO-MECHANICAL TESTING AND FOCUSED MONOCHROMATIC X-RAY DIFFRACTION................................ 71

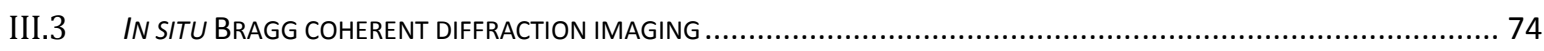

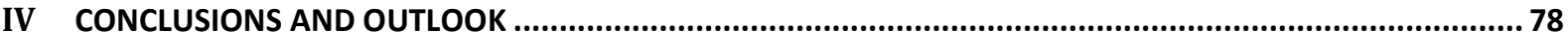

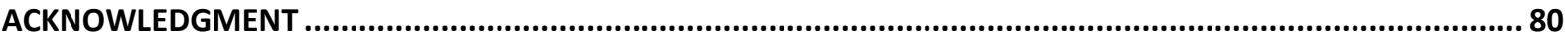

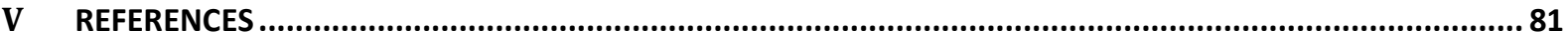




\section{ABSTRACT}

In recent years, the mechanical behavior of low-dimensional materials has been attracting lots of attention triggered both by the ongoing miniaturization and the extraordinary properties demonstrated for nanostructures. It is now well established that mechanical properties of small objects differ fundamentally from their bulk counterpart and in particular that "smaller is stronger" but many questions on the deformation mechanisms remain open. Most of the knowledge obtained on small- scale mechanics is based on ex-situ and in-situ characterizations using electron microscopy. However, these techniques suffer from the fact that imaging or scattering information is either limited to the surface or from a 2D projection of a thin foil of material. Within the last two decades tremendous progress was achieved at 3rd generation synchrotrons making it possible to focus hard X-ray beams down to the $100-\mathrm{nm}$ scale. Modern synchrotron X-ray diffraction methods may thus provide structural information with good spatial resolution and fully 3D. In this review, we discuss the progress achieved on in-situ micro- and nano-mechanical tests coupled with different synchrotron X-ray diffraction techniques to monitor the elastic and plastic deformation, highlighting the advantages of these approaches, which offer at the same time versatile sample environments and extreme precision in displacement fields. 


\section{INTRODUCTION}

The investigation of mechanical behaviour in small dimensions and the influence of nanostructuring on mechanical properties constitute very fundamental and active research topics [1, 2]. It is now well established that the mechanical behaviour of small objects differ fundamentally from bulk material and more specifically that "smaller is stronger", i.e. nanomaterials exhibit higher yield strengths. Many unsolved questions arise, however, concerning the strength of small (below $1000-100 \mathrm{~nm}$ ) objects. The role of dislocation nucleation and annihilation as well as the influence of atom diffusion at surfaces are still being debated. Within this review article we restrict ourselves mostly to monocrystalline metal and semiconductor micro- and nanostructures containing a limited number of defects while e.g. ultrafine grained materials are out of the scope.

The question of the influence of size on mechanical properties is certainly not new $[3,4,5]$ but the development of micro and nanotechnologies together with very advanced characterization and fabrication techniques has allowed harvesting a wealth of detailed information and exploring smaller scales. In the 1980s the field of mechanical properties of coatings was first pioneered by W. Nix and his co-workers in Stanford [6] where mechanical properties of thin films have been evaluated as a function of film thickness and microstructure. The fundamental issues raised by these studies as well as the imperious need to master the thermomechanical reliability of electron devices has been recognized by other research groups worldwide. We shall not try being exhaustive here but one should at least quote the groups of E. Arzt in Stuttgart [7], J. Harper in IBM Yorktown Heights [8], L. Freund at Brown University [9] and C. Thompson at MIT [10]. More recently, many research works focused on testing the mechanical properties of nanostructures (islands, nanowires, etc.). The development of focused ion beam (FIB) microscopes has allowed to fabricating sub-micron pillars out of bulk materials $[11,12]$. Compression tests performed on such objects $[11,12]$ have shown most of the time size effects. Tensile tests have also been reported [13]. The influence of defects induced by FIB-machining on these measured properties has been debated [14]. Mechanical testing of pillars prepared by other techniques such as direct deposition into pores [15] or wet etching $[16,17]$ indicate that similar size effects can be observed even in the absence of any FIB-machining depending on the internal dislocation content [18]. Tensile testing performed on single-crystal $\mathrm{Cu}$ nanowires [19] evidenced tensile strengths close to the theoretical limit. The majority of studies reported in the literature are performed on simple FCC metals $(\mathrm{Cu}, \mathrm{Ni}, \mathrm{Au}, \mathrm{Al})$. More recently, BCC metals have been studied [16] and also semi-conductors such as GaAs [20] or Si [21]. Because of deep Peierls valleys semi-conductors are brittle at room temperature. Surprisingly this is not true anymore in small dimensions: small diameter semiconductor pillars exhibit ductile behaviour [20]. 
The mechanical properties of nano-objects are being investigated by various ways. Tensile testing of $\mathrm{Cu}$ nanowires has been reported by Richter et al. [19]. Suspended nanowires have been bent with an AFM tip [22, 23, 24]. MEMS structures may also be used to perform tension tests [25]. These difficult experiments produce sometimes conflicting results and it is still difficult to decide whether this comes from the material under test or the test itself. The general trend is clear: smaller is stronger. On the other hand defect-free wires show weak size dependence and can be stressed until the theoretical shear strength. A single parameter like the flow stress does not, however, capture the full mechanical behaviour of these objects: when the size decreases the number of defects (dislocations, twins, etc.) is reduced and a stochastic behaviour is observed where sudden bursts occur in the stress-strain curve. Such behaviours call for in situ observation of defects and strain field during mechanical loading.

In situ observation of nano-objects during mechanical testing brings valuable information on the defects involved in the deformation process as well as on the local strains. Scanning electron microscopy coupled with tensile or compressive testing $[12,13,20,26]$ allows one to look at the change in sample shape as well as visualize the appearance of slip traces at the sample surface. In situ transmission electron microscopy is more challenging and necessitates special sample preparation but has the distinct advantage of visualizing individual dislocations movement $[27,28$, $29,30]$. X-ray diffraction, on the other hand, has the benefit of being non-destructive, penetrating (hence no special need for pre-thinning the sample) and extremely sensitive to elastic strains and defects. Thanks to the development of new sources (synchrotron facilities), increasingly efficient focusing optics and advanced $2 \mathrm{D}$ detectors $\mathrm{X}$-ray diffraction is becoming a major tool for investigating the structure of nano-objects in situ during mechanical testing.

Diffraction of X-rays by crystals was first evidenced by M. Laue [31] in 1913 using a white X-ray beam and a photographic film. This white beam diffraction setup now bears the name of its first inventor. Soon after Laue's discovery it was realized that Laue diagrams from deformed crystals show distinct elongated features called asterism $[32,33]$. Hence, the sensitivity of $X$-ray diffraction to crystal imperfections was realized even before the concept of edge dislocation was introduced independently by Taylor [34], Polanyi [35], and Orowan [36] in the early 1930s. Elastic strains and related stresses can be deduced with great accuracy from the shift of Bragg peaks $[37,38]$. This approach still remains very effective in measuring elastic strains in various materials [39]. Strain imaging with X-rays has made very impressive progress lately. On the one hand, progress in X-ray focusing optics allows nowadays scanning X-ray diffraction mapping to be performed with a resolution in the $50-100 \mathrm{~nm}$ range $[40,41]$. Full field X-ray microscopy is improving a lot too with resolutions in the $100 \mathrm{~nm}$ range [42]. By far the best spatial resolution, on the other hand, is 
obtained with coherent diffraction imaging, which is a lens-less imaging technique, with a typical resolution of 8-10 $\mathrm{nm}[43,44]$.

These recent progresses which have been made possible thanks to the development of brighter and more coherent synchrotron sources are revolutionizing the field of mechanics at small scales. We think the time is ripe for a review of this rapidly moving field, which will develop a lot in the coming years thanks to the new sources that are presently developing. The purpose of this review is to give the reader the tools necessary to understand what can be obtained with a combination of nanomechanical testing and synchrotron X-ray diffraction. In addition we are giving here the state of the art of what can be performed at the end of 2017 in this field. The review starts with a survey of nanomechanical testing methods and then follows with some basics about X-ray diffraction. Finally, we present an overview of what has been achieved so far with various combination of in situ X-ray scattering and nano-mechanical testing.

For further reading, good overviews over tensile testing on micro- and nanostructures can be found in the review articles by Gianola and Eberl [45] and by G. Dehm [46]. Furthermore, the combination of in situ mechanical tests with X-ray diffraction was reviewed by Van Swygenhoven and Van Petegem [47]. This article is complementary to the present overview since these authors focused on tensile tests on polycrystalline samples coupled with powder X-ray diffraction and on microcompression studies in combination with Laue microdiffraction. 


\section{MECHANICAL TESTING AT SMALL SCALES}

The analysis of the mechanical behavior of materials involves two aspects: i) the determination of stress and strain state in the loaded system which is never homogeneous at the microstructural or defect scale, and homogeneity or heterogeneity at overall scale depending on loading configuration and geometry; ii) the determination of the mechanical properties which, themselves, link how stress evolves with strain and which also allow ranking materials.

\section{I.1 Basics about mechanical properties}

Besides many other physical properties, the determination of the mechanical behavior of materials is of uttermost importance for the reliability of any kind of devices. In general, the mechanical response of any piece of solids is described by a reversible (elastic) and an irreversible (plastic or fracture) part. The relative amount of both quantities determines the deformation behavior of the material. The elastic part is described by Hooke's law where the slope of the stressstrain $(\sigma-\varepsilon)$ curve, which is schematically depicted in Fig. 1, is the Young's modulus $E$ of the material $(\sigma=E \cdot \varepsilon)$. When deforming a material beyond its elastic limit (yield strength), the plastic regime starts involving irreversible defects such as the nucleation or glide of dislocations.

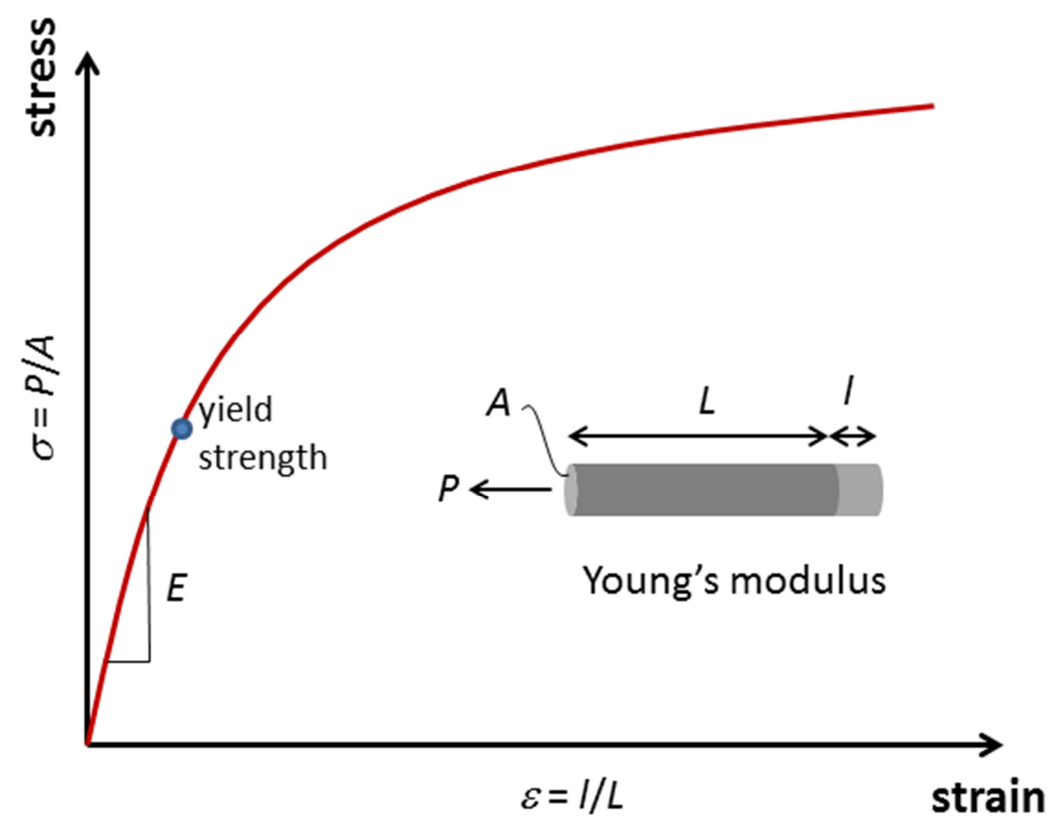

Fig. 1: Schematic stress-strain curve for a tensile test on a structure with length $L$ and cross-sectional area A.

The presence of mechanical stress in any kind of device may eventually lead to failure and, as such, is intimately related to reliability and process yield. Considering the fact that the sizes of the building blocks of nowadays electronics are further and further reduced thanks to the ongoing 
miniaturization the mechanical behavior of low-dimensional materials got into the focus of scientists all over the world. When performing a gedankenexperiment assuming a certain dislocation density in a bulk material and then reducing the size of the object one finally ends up with a structure of few or even zero dislocations (see Fig. 2). Thus, from a certain size on, it is difficult to speak about dislocation densities since the structure in total may exhibit one or two or zero dislocations. Consequently, the mechanical behavior on this size scale is expected to become stochastic clearly deviating from the bulk behavior.
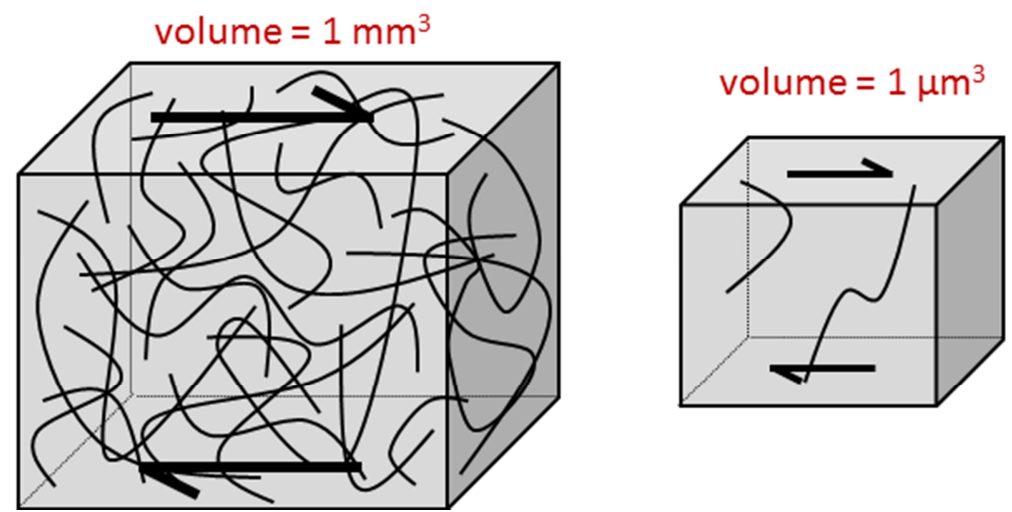

volume $=100 \mathrm{~nm}^{3}$

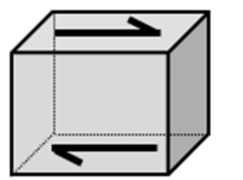

Fig. 2: Schematic illustration of the gedankenexperiment on the evolution of dislocation density in materials when reducing the sample size.

While bulk metals contain many pre-existing dislocations and dislocation sources resulting in yield strengths of the order of few megapascal, the absence of dislocations increases the necessary stress to introduce a dislocation enormously. Frenkel showed that the yield strength for dislocationfree crystals is of the order of one tenth of the elastic shear modulus $\mu(\sigma \sim \mu / 10)$, i.e. a few gigapascal. In 1952, Herring and Galt reported yield strengths for Sn microwhiskers close to the Frenkel theoretical limit $[4,48]$. A similar behavior was evidenced on $\mathrm{Fe}, \mathrm{Cu}$, and $\mathrm{Ag}$ microwhiskers by S.S. Brenner a few years later [5]. This unexpected high yield strength is attributed to the lack of preexisting dislocations in the nanostructures.

\section{I.2 Micro- and nano-mechanical testing methods}

In order to study the mechanical behavior at the micro- and the nano-scale major efforts have been undertaken to develop various ex situ and in situ mechanical testing setups ranging from curvature measurements of thin films over local testing methods such as nano-indentation to uniaxial as well as bending methods. The goal is both to evaluate stress and strain values at overall or local scale and to extract mechanical properties. Here, firstly different testing methods are reviewed before introducing ex situ micro-mechanical tests, followed by recent developments on in situ testing apparatuses for micro- and nano-mechanical studies. 


\section{a) Curvature measurement}

Many of today's high performance devices based on thin films rely on residual stresses within the individual layers which originate from the lattice mismatch, the different thermal expansion coefficients of the different materials, and the growth itself. For achieving the desired optical, electronic and mechanical properties it is critical to understand and to control the stresses in thin films during thermal annealing processes. The most commonly used method for studying the residual stress in such thin films is the measurement of the substrate curvature by monitoring the deflection of a laser beam that is reflected by the sample surface and focused on a two-dimensional detector (Fig. 3). When using a 2D laser beam array the sample deformation can be determined in two dimensions.

By using the known geometry (incident angle $\alpha$ of the laser beam and sample-detector distance $L$ ) and material properties of the substrate, the film stress is determined during heating cycles by measuring the differential beam spacing $\delta \mathrm{d} / \mathrm{d}_{0}$ caused by a bending of the film/substrate stack due to differences in film stress and thermal expansion coefficient. The high resolution of the differential beam spacing $\delta d / d_{0}$ ensures the high measurement sensitivity of about $6 \cdot 10^{-5} \mathrm{GPa} \cdot \mu \mathrm{m}$ stressthickness product $[49,50,51]$. Measuring the thermoelastic slope in a supported thin film yields the product of biaxial modulus by the difference in the coefficient of thermal expansion (CTE) between film and substrate. Isothermal experiments provide kinetics of stress relaxation and estimates of the activation volume in case of dislocation processes [52]. While these types of measurements are comparatively easy to perform, it is rather complicated and cumbersome to decouple the stress from the temperature.

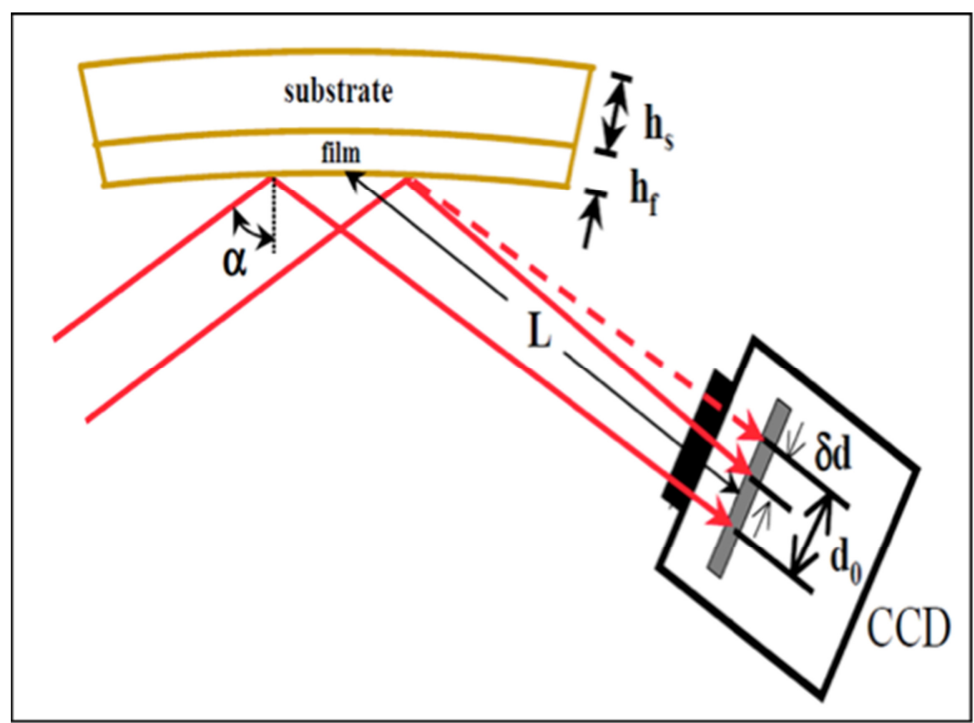

Fig. 3: Schematic drawing of the curvature measurement on a thin film with a thickness $h f$ deposited on a substrate with thickness hs by laser beam deflection (from ref. [53]). The displacement $\delta d$ of the laser beam on the detector is directly related to the curvature of the thin film from which the film stress is calculated using the Stoney equation. 


\section{b) Nano-indentation}

A very good review on nano-indentation and on recent developments in this field can be found in ref. [54]. Thanks to its simplicity and speed, indentation became a very common technique to locally measure the mechanical properties of materials. At the beginning of last century, Brinell conducted first indentation tests employing spherical and smooth balls from ball bearings as indenters to measure the plasticity of materials $[55,56]$. This kind of test (so-called Brinell test) was rapidly accepted by industry as testing method soon after its introduction and stimulated the development of various macro- and micro-indentation tests [57]. Traditionally, the indent is imaged by optical microscopy which evidently imposes a lower limit on the resolution and thus on the length scale of indentation. More recently, indentation testing was extended down to the nanoscale.


Fig. 4: Schematic illustrations a) of indentation load-displacement data with a peak indentation load $\boldsymbol{P}_{\max }$, an indenter displacement $h_{\max }$ at peak load, a final indentation depth $\boldsymbol{h}_{\mathrm{f}}$ and an initial unloading stiffness $S$. b) Schematic representation of a section through an indentation showing the contact radius a, the opening angle $\phi$ of the indenter tip, the total displacement $h$, the contact depth $h_{\mathrm{c}}$, the amount of $\operatorname{sink}$-in $h_{\mathrm{s}}$, and the permanent depth of penetration $h_{\mathrm{f}}$ after complete unloading [58]. 
Indentation load-displacement data contain a wealth of information (Fig. 4(a)). From the loaddisplacement data, many mechanical properties such as hardness and elastic modulus can be determined without imaging the indentations $[56,59]$ As the indenter is pressed into the sample, both elastic and plastic deformation occur, which results in the formation of a hardness impression conforming to the shape of the indenter (Fig. 4(b)). During indenter withdrawal, only the elastic portion of the displacement is recovered, which facilitates the use of an elastic solution in modeling the contact process $[56,59,60]$.

Nano-indentation hardness is defined as the indentation load divided by the projected contact area of the indentation. It is the mean pressure that a material can support under load. The elastic modulus of the indented sample can be inferred from the initial unloading contact stiffness, $S=$ $\mathrm{d} P / \mathrm{d} h$, i.e., the slope of the initial portion of the unloading curve. Hereby the contact between the indenter and the sample under study is described by a Hertzian contact model which was firstly introduced by Heinrich Hertz in 1882 [61] and further improved by Sneddon in 1965 [62]. To calculate the elastic modulus $E$, the contact stiffness and the projected contact area need to be determined from the load-displacement curve. While Doerner and Nix [63] suggested a linear unloading curve, Oliver and Pharr [59] found that the unloading curve is better described by a power law

$$
P=B\left(h-h_{f}\right)^{m}
$$

where $B$ and $m$ are empirically determined fitting parameters.

Recently, a new technique, the continuous stiffness measurement (CSM) $[59,64,65]$ technique has been developed which allows for accessing the contact stiffness and, thus the elastic properties of the tested material during the loading portion of an indentation test. The CSM is accomplished by imposing a small, sinusoidal signal on top of a DC signal that drives the motion of the indenter. The response of the system is analyzed by means of a frequency specific amplifier allowing for the measurement of contact stiffness at any point along the loading curve and not just at the point of unloading as in the conventional measurement. The CSM technique makes the continuous measurement of mechanical properties of materials possible in one sample experiment without the need for discrete unloading cycles, and with a time constant that is at least three orders of magnitude smaller than the time constant of the more conventional method of determining the stiffness from the slope of an unloading curve. 


\section{c) Uniaxial (compression and tensile) tests}

Uniaxial (compression and tensile) tests (Fig. 5) induce less complex strain fields and are thus easier to interpret. Compression experiments are usually carried out in load- or displacementcontrolled mode, with a nano-indenter equipment where the sharp indenter has been flattened to a flat punch.

The goal is mainly to extract the low and large strain plastic behavior. Tensile tests on the contrary are useful to reach fracture on top of determining elastic and plastic response. While compression tests are limited to structures with aspect ratios (height vs. diameter) of less than three to avoid any buckling during the mechanical loading, tensile tests allow for testing much longer specimen. However, in both cases, the alignment of the mechanical tester with the structure is crucial and, in particular for low-dimensional materials, it is a major complication. In case of misalignments shear strain may affect the mechanical response. For compression experiments the tester head and the upper surface of a pillar-like structure have to be parallel to avoid any shear forces. However, when plasticity occurs the structure cannot move freely due to the friction on the tester head. Thus, the mechanical response may be affected by the experimental setup.

a

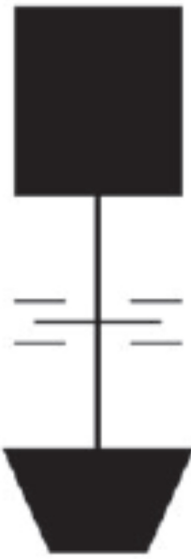

Capacitive displacement sensor

Diamond flat punch

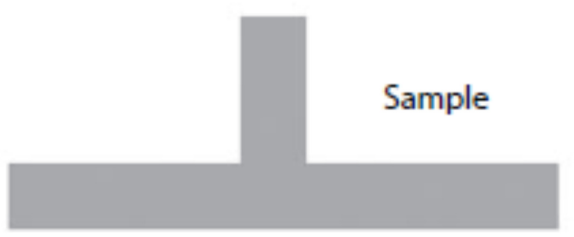

Force Measurement

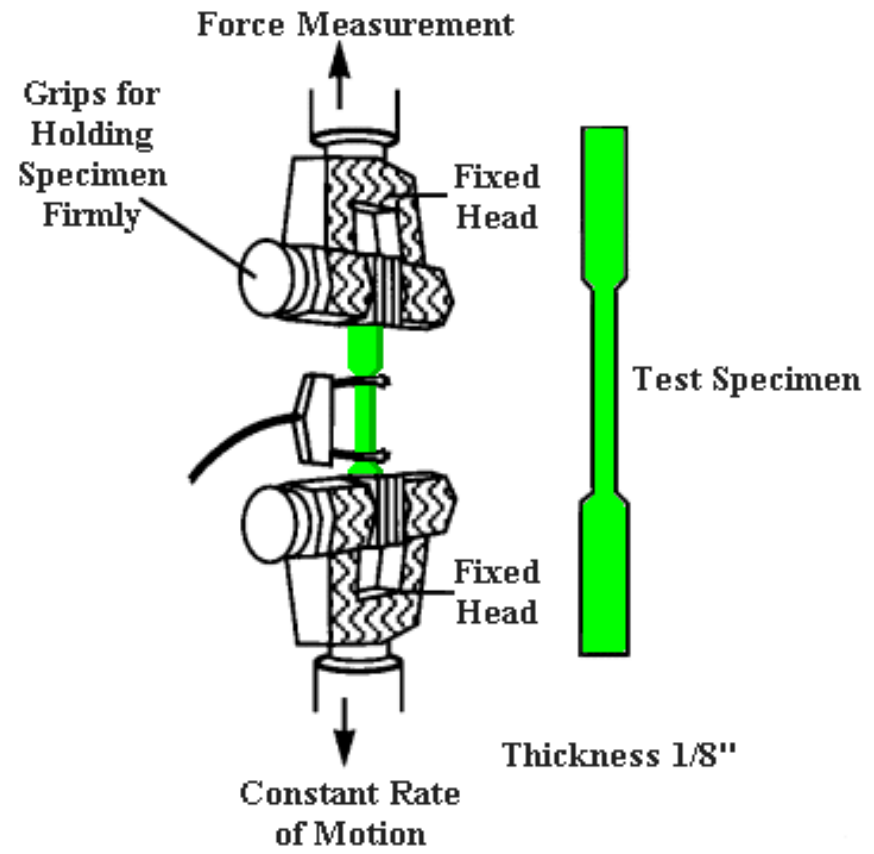

Fig. 5: Schematics of uniaxial compression [66] and tensile tests [67].

\section{d) Bending}

Bending experiments, which are mainly used for studying the elastic behavior and small plastic deformations, may be performed in various configurations, which are schematically illustrated in Fig. 6. On the one hand, the structure to be tested may be clamped on one side only and the load is 
applied at its free end resulting in a cantilever-like test. On the other hand, both ends of the testing structure may be supported and the load is applied on one or two points along the suspended structure facilitating three-point and four-point bending-tests. For the mechanical behavior of the tested structure, the boundary conditions, i.e. whether the structure is simply supported or thoroughly clamped at its ends is of uttermost importance. While uniaxial loadings result in comparatively simple strain distributions within the test structure, bending results in inhomogeneous strain fields within the specimen.

a)



b)
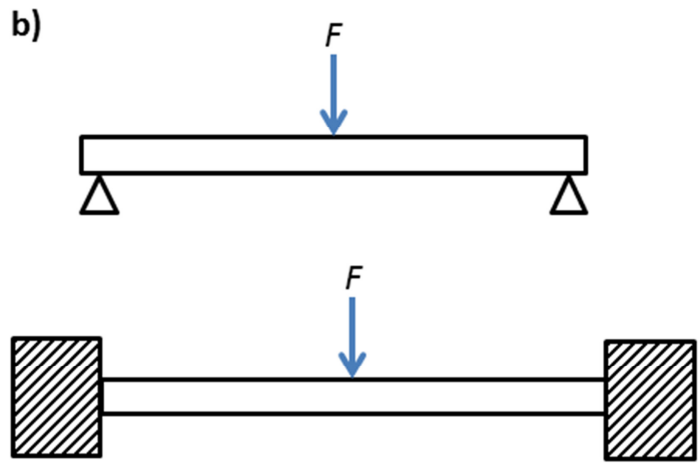

c)
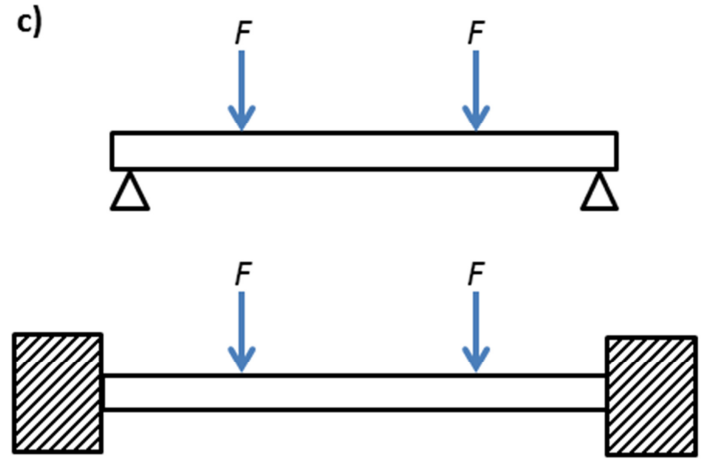

Fig. 6: Schematics of bending tests in a) cantilever configuration, b) in three-point bending and c) in fourpoint bending configuration for a simply supported and a doubly clamped beam.

Considering a slender beam which is homogeneous along its length $L$, the governing equation for deriving the bending of the beam is the following

$$
E I \frac{d^{4}(\Delta z)}{d x^{4}}-T \frac{d^{2}(\Delta z)}{d x^{2}}=F \cdot \delta(x-L)
$$

where $E$ is the material's Young modulus, $F$ is the applied force, $I$ is the area moment of inertia of the cross-section, $\Delta z$ is the deflection of the beam, $x$ is the spatial coordinate along the beam length, and $T$ is the tension along the beam. For deflections smaller than the half-thickness of the beam, the second term on the left hand side can be neglected and one obtains the well-known Euler-Bernoulli equation which can be solved for the various boundary conditions such as simply supported, double clamped, point load, or homogeneous load. The formulas of the deformation $\Delta z$ for the most common configurations for bending tests on nanostructures, namely three-point bending on simply supported and double clamped beams, are presented below: 


$$
\begin{aligned}
& \text { simply supported: } \Delta \mathrm{z}_{\text {center }}=\frac{F L}{48 E I} \\
& \text { double clamped: } \Delta \mathrm{z}_{\text {center }}=\frac{F L}{192 E I}
\end{aligned}
$$

Note that for larger deflections than the half thickness of the beam, tensile strains along the tested structure evolve making it necessary to take into account higher orders complicating the calculation of the bending profile. In this large deflection regime there is not anymore a neutral fiber.

\section{e) Vibration tests}

Besides all the static testing methods, the elastic behavior may also be studied dynamically. For this purpose, a cantilever-like specimen is clamped at one of its ends or the structure is clamped at both ends and vibrated at its resonance frequency $f_{\text {res }}$ giving access to the Young's modulus (Fig. 7). The following equations describe the fundamental mode of the resonance frequency for single and double-clamped nanowires where $E$ is the Young's modulus, $\rho$ is the mass density, $r$ is the radius for a cylindrical nanowire, and $L$ is the nanowire length [68].

$$
\begin{gathered}
\text { single clamped nanowire: } f \approx 0.28 \sqrt{\frac{E}{\rho}} \frac{r}{L^{2}} \\
\text { double clamped nanowire: } f \approx 1.78 \sqrt{\frac{E}{\rho}} \frac{r}{L^{2}}
\end{gathered}
$$

The structure may be excited either mechanically or by magnetic fields depending on the material or thermally [69]. Note that an additional mass on the vibrating structure affects the resonance frequency making this technique highly sensitive to attached matter and, thus, it may be employed for detecting small amounts of matter. On the other hand, contaminations on the structure also affect the resonance frequency and, thus the measurement of Young moduli making this approach rather vulnerable.

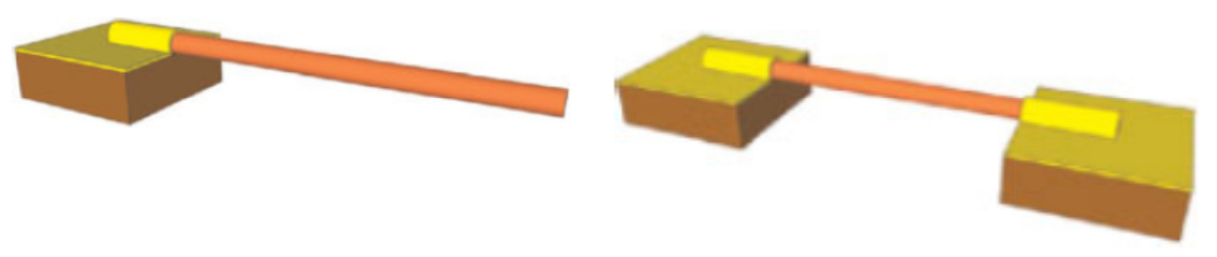

Fig. 7: Scheme for vibrational tests on a) a single clamped and b) a double clamped nanowire [68]. 


\section{I.3 Recent results from ex-situ micro and nano-mechanical tests}

In the 50s of the last century, S.S. Brenner conducted pioneering works on micro-whiskers demonstrating extraordinary mechanical behaviors on the micron scale [5]. Tensile tests on iron, copper, and silver whiskers with diameters ranging from 1.2 to $15 \mu \mathrm{m}$ and lengths of up to $4 \mathrm{~mm}$ demonstrated strengths which were close to the strength of perfect crystals. In addition, Brenner showed that the strength decreased with increasing diameter and length which he attributed to defects which were formed during the growth. Since then great progress has been made on the instruments necessary to thoroughly study the mechanical behavior on the micro- and nano-scale as will be demonstrated below.

\section{I.3.i Micro-compression tests}

With the advent of focused ion beam (FIB) microscopes a new era of micro- and nanomechanical testing was launched. With this microscope, it became feasible to machine out of bulk materials specimens of various size and shape dedicated for micro-mechanical testing as schematically illustrated in Fig. 8. Uchic et al. pioneered the field of micro-compression tests on FIB milled micropillars [11]. The fabrication of pillars usually starts from a bulk material with a flat surface. Material is milled away with the ion beam to leave a cylindrical column at the center of an excavation of free space. This crater has to be sufficiently large in diameter in order to avoid the contact of the indenter head used for compression tests with the surrounding material [70]. The key difficulty is the perfect parallelism between the flat punch and the specimen.
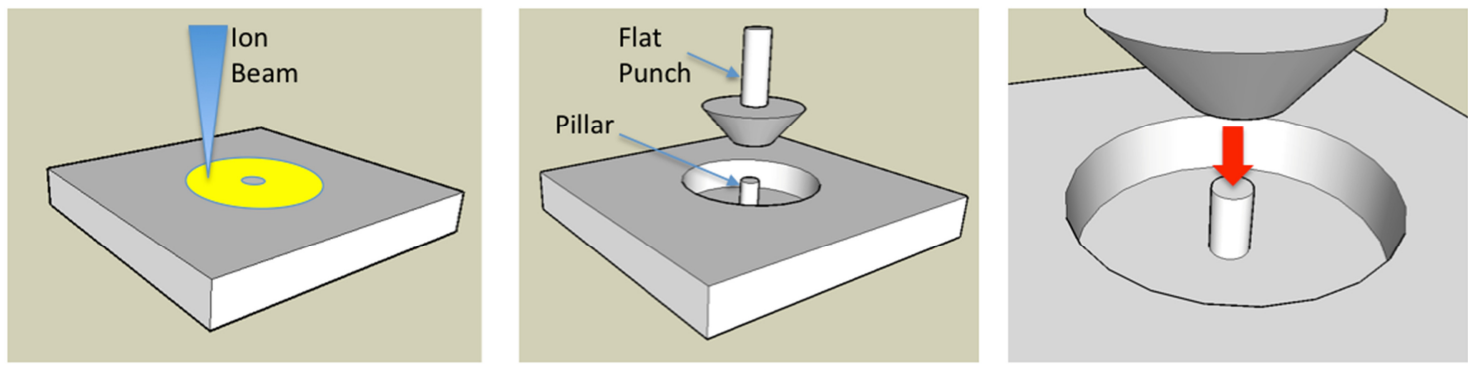

Fig. 8: Principle of a nano-compression experiment on a pillar carved in a flat surface. a) The initial sample can be a bulk material with a flat surface. Once the pillar is carved by FIB milling, b) the flat punch indenter is optically aligned and c) the test can be carried out in displacement or load control [70].

When using a single ion beam direction for the milling process, the resulting pillars exhibit tapered flanks due to the ion beam defocusing along their trajectory. However, by inclining this tapered pillar almost perpendicular to the ion beam the taper may be milled away leaving a perfectly cylindrical micropillar as shown in Fig. 9(a) [66]. The circle milled on the top of the pillar serves as a reference in image recognition processing software to perform the off-axis rotation automatically. 
Fig. 9(b) shows a SEM image of the same pillar as in part (a) after micro-compression testing revealing glide planes. The pillar was oriented along the [269] direction and, thus, it was single slip oriented. In conclusion, FIB milling allows for creating dedicated specimen for micro-compression testing. However, one should keep in mind that the FIB milling process bases on the irradiation of the target material with $\mathrm{Ga}^{+}$ions sputtering off target atoms. This process may lead to an amorphization of the sample surface as well as to an implantation of $\mathrm{Ga}^{+}$ions in a near surface region of the specimen. In addition, milled material is re-deposited in the surroundings of the specimen $[71,72]$.

Fig. 9(c) presents the stress-strain curves for Ni micropillars with diameters ranging from 5 to 40 $\mu \mathrm{m}$ as well as for bulk nickel [11]. While for bulk material the stress-strain curve is smooth the stressstrain curves for the micropillars show strain bursts which become more frequent with decreasing pillar diameter. Moreover, the yield strength increases significantly as the pillar diameter is reduced. However, the yield stress differs from one pillar to another with the same diameter, showing the critical influence of the initial microstructure at small dimensions and emphasizing the need for statistical studies [73]. In addition, hardening tends to appear as the pillar diameter decreases.
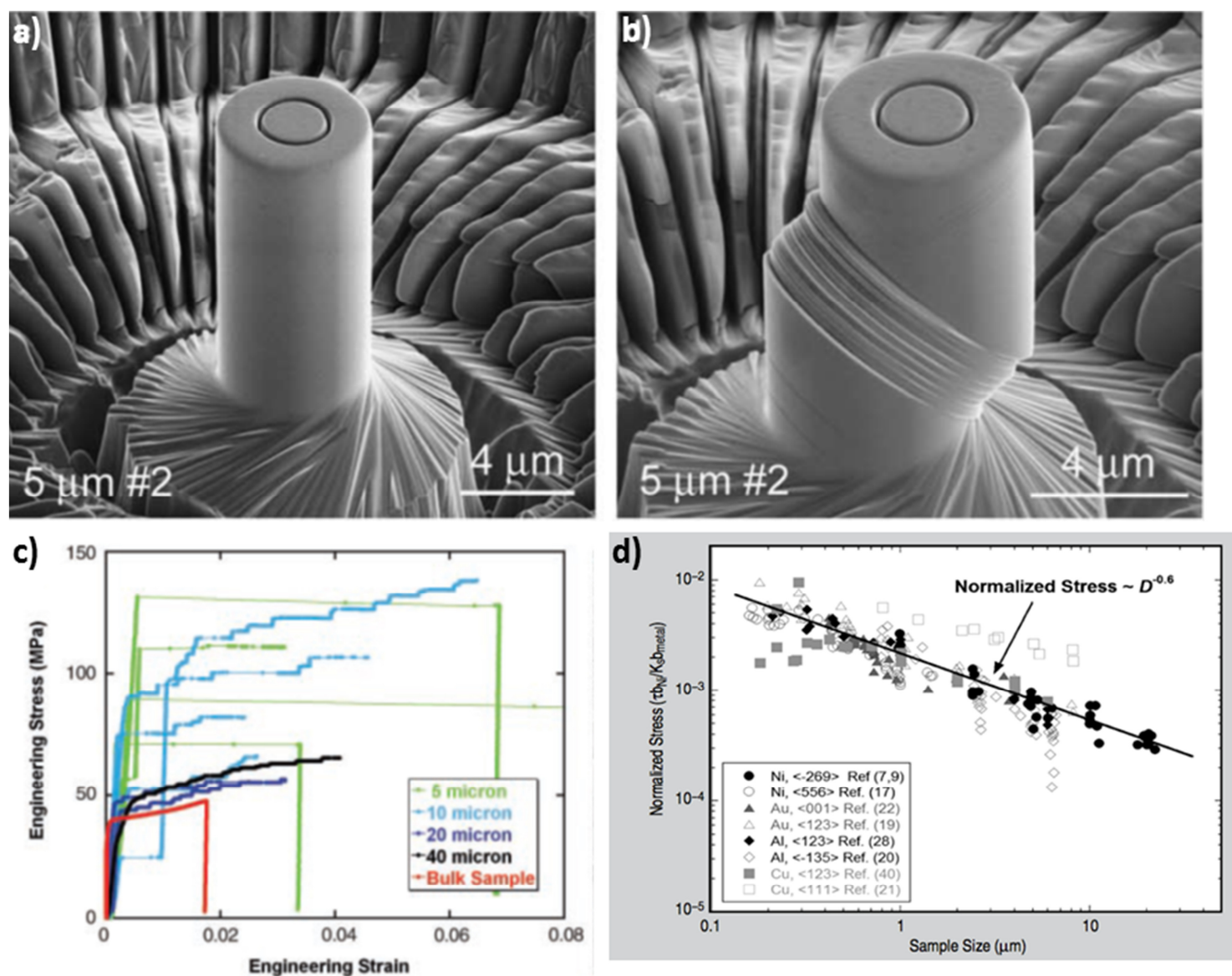

Fig. 9: SEM images of a FIB milled Ni micropillar a) before and b) after micro-compression testing [66]. c) Engineering stress-engineering strain curves for FIB milled Ni micropillars of different diameters [11]. d) Log-log plot of yield stress as a function of structural size for FIB milled FCC metal pillars [74]. 
Numerous compression experiments on micropillars of various FCC metals ( $\mathrm{Ni}, \mathrm{Au}, \mathrm{Al}, \mathrm{Cu}$ ) have been performed over the past years all showing the same trend that the yield stress increases with decreasing pillar diameter (see Fig. 9(d)) $[74,75,76,77,78]$. This trend became known in literature as "smaller is stronger". The yield stress as a function of the pillar diameter can be described by the following equation

$$
\sigma=\sigma_{0}+k d^{-n}
$$

where $d$ is the pillar diameter and $k$ and $n$ are fitting parameters. Here, the best fit is obtained for $n=$ 0.6 .

Very recently, J.A. El-Awady performed three-dimensional discrete dislocation dynamics (DDD) simulations, building on an earlier suggestion by Lilleodden et al. [79], which supports the existence of a well-defined relationship between the strength and the dislocation microstructure at all length scales for both single-crystalline and polycrystalline materials [80].The results show a correlation between crystal strength and dislocation density for micron and sub-micron crystal sizes, and a minimum crystal strength marked by a transition from dislocation-source strengthening to forestdominated strengthening at a size-dependent critical dislocation density. Fig. 10 shows a composite plot of the resolved shear strength $\tau$ normalized by the shear modulus $\mu$ as a function of the initial dislocation density $\rho$ for DDD simulated Ni single crystals having diameters in the range of 0.25 to 20 $\mu \mathrm{m}$. For each crystal size, the strength scales with the dislocation density following a power-law of the form $\tau=\rho^{n}$. Four deformation mechanisms can be identified as a function of the crystal size and the initial dislocation density: (i) dislocation starvation, (ii) single-source mechanism, (iii) exhaustion hardening, and (iv) forest hardening [80]. 


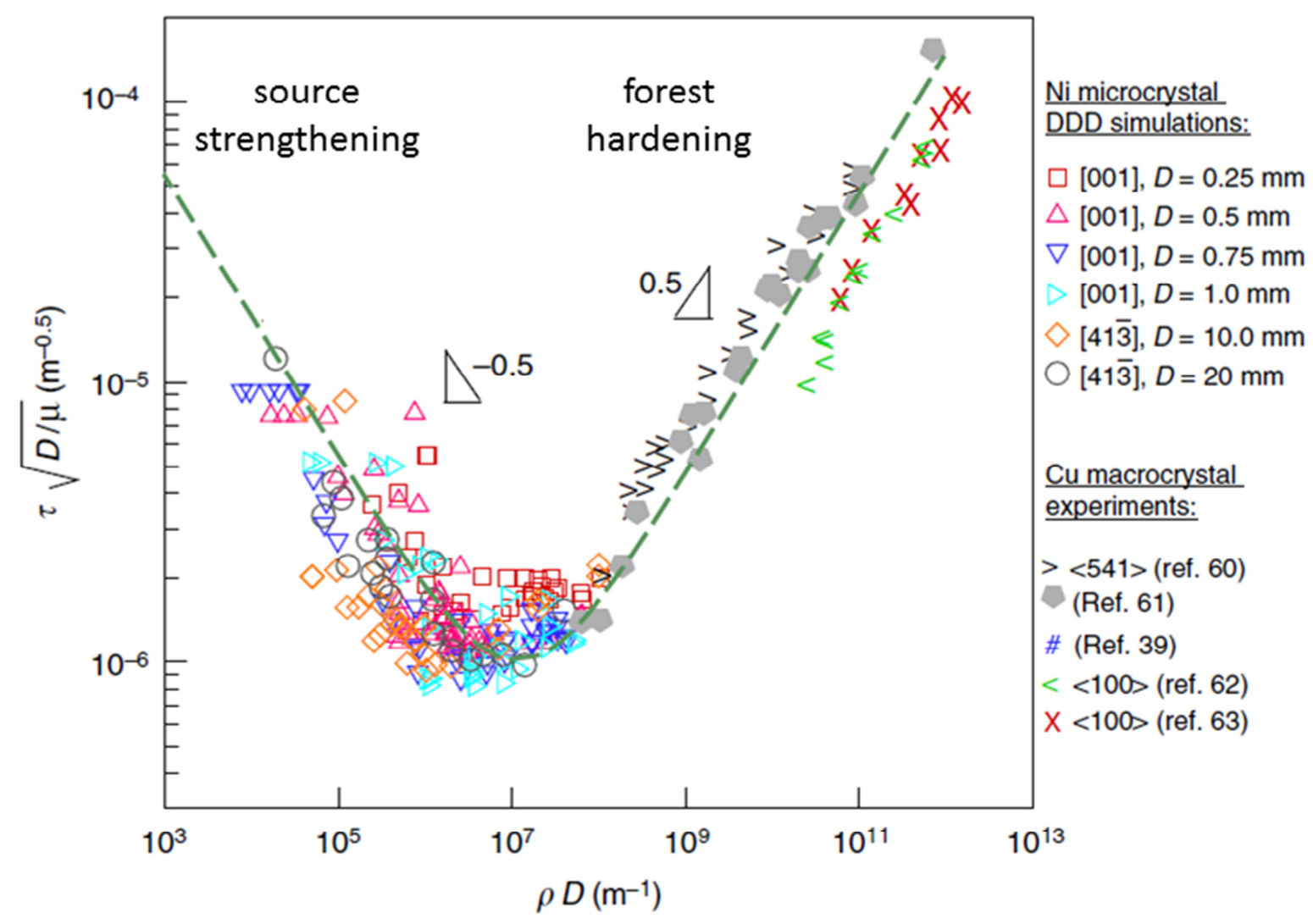

Fig. 10: Composite plot of the dimensionless-resolved shear strength multiplied by the square root of the crystal diameter versus the initial dislocation density multiplied by the crystal diameter from DDD simulations of microcrystals in the range $0.25 \leq D \leq 20 \mu \mathrm{m}$ and experiments on Cu macrocrystals [80].

In contrast to FCC metals, the dislocation processes in BCC metals differ fundamentally. The dislocation motion occurs on various slip systems with screw dislocations being slower than edge dislocations due to the non-planar core structure and associated need to overcome the Peierls potential by thermal activation. This fundamental difference of plasticity in BCC compared to FCC metals results also in a different size dependence of the mechanical behavior of BCC micropillars. Schneider et al. demonstrated by microcompression tests on FIB milled BCC micropillars that the relationship between yield strength and pillar diameter correlate with a critical temperature $T_{\mathrm{c}}$ below which screw dislocations are less mobile than edge dislocations [81]. The mechanical size effect for different BCC micropillars is shown in Fig. 11(a) together with data for FCC Au micropillars for comparison. The slope which corresponds to the exponent $n$ in equation (5) increases from 0.21 for $\mathrm{W}$ to 0.48 for $\mathrm{Nb}$ always being smaller than 0.6 for $\mathrm{Au}$. The exponent is plotted as a function of the normalized test temperature $T / T_{\mathrm{c}}$ in Fig. 11(b) showing a linear relationship. The extrapolation (dashed line) to lower critical temperatures yields an exponent of $\sim 0.6$ which corresponds to a material where screw and edge dislocations have the same mobility (FCC-like behavior). Thus, the small-scale mechanical behavior for BCC metals depends both on size and on temperature. 

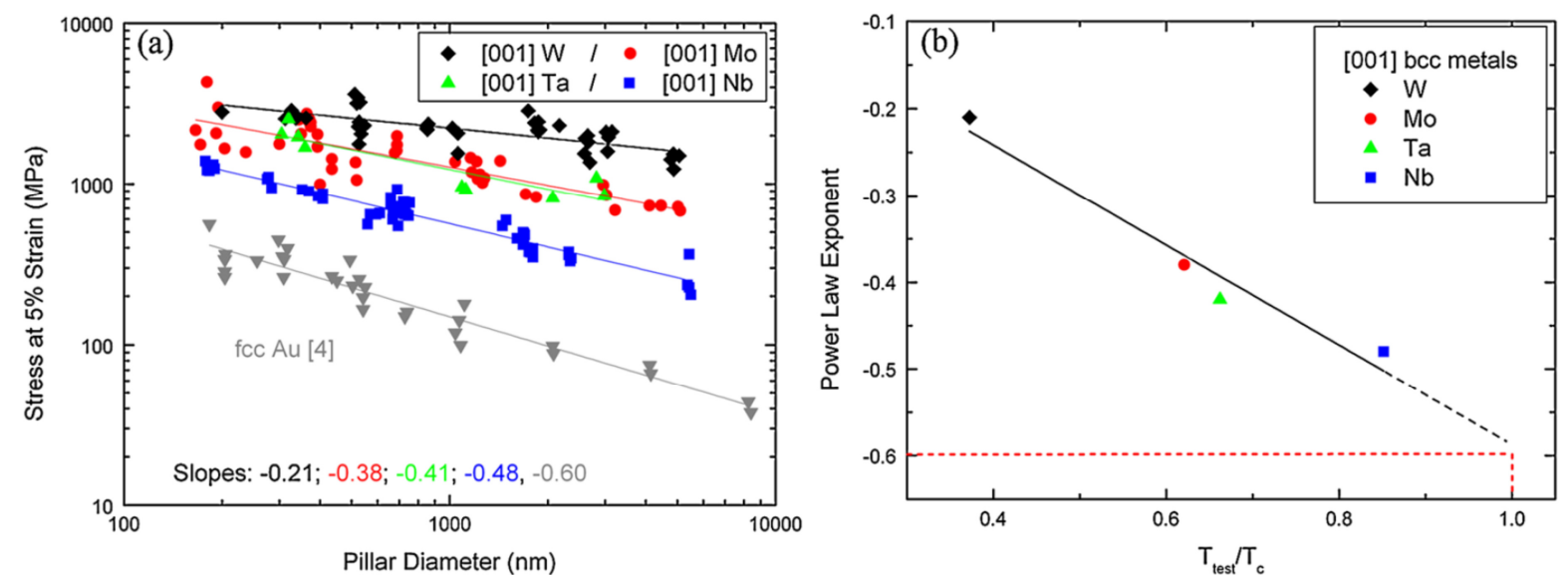

Fig. 11: a) Log-log- plot of stress measured at 5\% strain versus pillar top diameter for [001] BCC pillars (W, Mo, Ta, Nb) as well as [001] Au pillar data [4]. (b) Slope of the line of best fit (exponent) for the size dependence of the BCC metals shown in (a) versus test temperatures normalized with respect to the critical temperature $T_{\mathrm{c}}$ which defines the temperature at which edge and screw dislocations have the same mobility [81].

As already mentioned before, the FIB milling process is based on the irradiation of the target material with $\mathrm{Ga}^{+}$ions which may amorphize the sample surface and may implant $\mathrm{Ga}^{+}$ions in a near surface region of the specimen. The influence of these sample modifications on the mechanical properties were debated within the scientific community. First indications that ion beam milling may affect the mechanical behavior of micro-structures were revealed by compression tests on asprepared Mo-rich fibers embedded in a Mo-Ni $\mathrm{N}_{3} \mathrm{Al}$ alloy. These fibers with diameters ranging from 300 $\mathrm{nm}$ to $3 \mu \mathrm{m}$ are initially free of dislocations. Pillars for micro-compression testing were prepared by etching the alloy matrix, thus, freeing the upper part of the fibers. The scanning electron micrograph in Fig. 12(a) presents the square and faceted pillars where the central one has been mechanically deformed. Independent of the fiber diameter the yield strength of the as-prepared pillars was found to be $99 \mathrm{GPa}$ being close to the theoretical limit of the material [16]. The introduction of dislocations before micro-compression tests by pre-straining Mo alloy pillars with diameters ranging from 360 to $1500 \mathrm{~nm}$ evidenced lower yield strength compared to as-prepared pillars [82]. A similar behavior was reported for Mo alloy micropillars after being irradiated with a $\mathrm{Ga}^{+}$ion beam in a FIB microscope where the yield strength dropped by one order of magnitude [18]. White beam Laue microdiffraction on as-prepared Mo pillars as well as on the same type of pillars after FIB milling and pre-straining evidenced deviatoric strain gradients and/or orientation gradients for the treated microstructures [83]. These Laue results provide an explanation for the loss of the whisker-like mechanical behavior of directionally solidified Mo pillars. Similar results were found for etched and FIB-milled Si pillars where the streaking of the Laue spots for the FIB prepared pillars may indicate anisotropic strain distributions or contribution of excess dislocations or rotational effects which may originate from the 
incorporation of $\mathrm{Ga}^{+}$ions or the recrystallization of surface amorphous layer induced by the FIB milling [14]. Kiener et al. studied the influence of ion energy, ion dose, and the incidence angle of a $\mathrm{Ga}^{+}$ion beam on a Cu sample by TEM and Auger electron spectroscopy (AES) [84]. Conventional TEM studies reveal the necessity to reduce the $\mathrm{Ga}^{+}$damage by reducing the ion energy, milling current, and incidence angle, if pre-existing defects are to be analyzed. Gallium concentrations as large as $\sim 20$ at.\% were found close to the surface of polycrystalline Cu by AES. Thus, these experimental findings evidence that FIB milling may modify the specimen in a near surface region affecting the mechanical behavior on the micro- and the nano-scale. The effect of ion beam milling on the mechanical properties may be reduced by lowering the acceleration voltage and, thus, the penetration depth of the $\mathrm{Ga}^{+}$ions into the material.
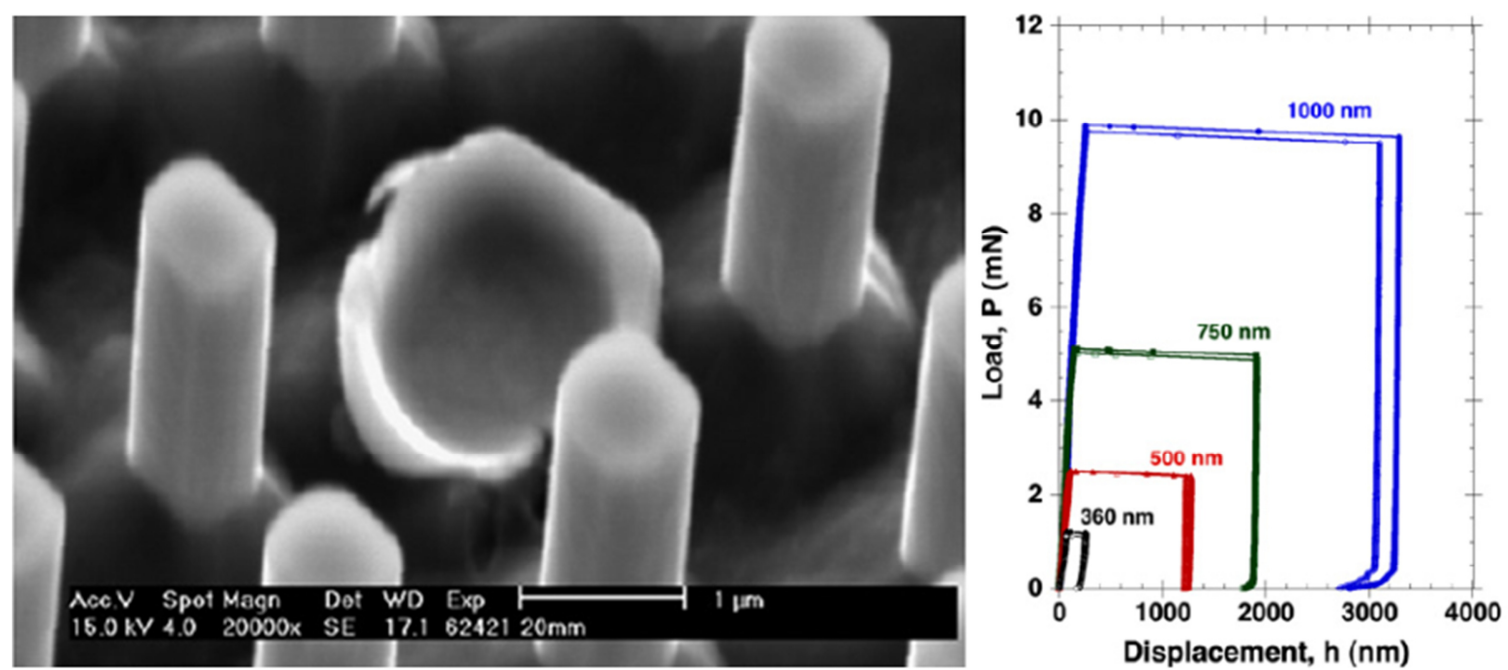

Fig. 12: Scanning electron micrograph of molybdenum alloy micro-pillars fabricated by selective etching of the matrix in a NiAl-Mo directionally solidified eutectic and compressive load-displacement curves for micro-pillars with different diameters [16].

As mentioned above Laue microdiffraction allows for investigating the volume of the structures while SEM is a surface sensitive tool. Therefore, microstructures which were mechanically deformed in situ in a SEM chamber were subsequently investigated by Laue microdiffraction to gain additional information on activated dislocations. While in situ SEM observations only showed predicted slip planes with the highest Schmid factor intersecting the sample surface, post mortem Laue microdiffraction experiments evidenced that the sample base and head did not deform in any way. In addition, Laue microdiffraction revealed dislocations of an unpredicted slip system at low strains which were not able to escape to the surface in larger numbers and, thus no similar slip steps were observed on the sample surface. Moreover, Laue microdiffraction experiments by Kirchlechner et al. showed that multiple slip takes over at later deformation states [85]. Results obtained by Laue microdiffraction will be reviewed and discussed in further detail in section III.1. 


\section{I.3.ii Bending tests on nanowires}

Scanning probe microscopes provide information on surface morphology and they allow for a local characterization of a wide number of physical properties with a resolution going down to the atomic scale. In particular, atomic force microscopes give direct access to force and displacement and, thus, they are pre-destined instruments for mechanical tests at the nanoscale. It allows for bending structures in cantilever or in three-point configurations where the object is clamped either on one end only or it is placed across holes or trenches forming doubly-clamped self-suspended bridges [23, 24]. In 2000, Cuenot et al. successfully deduced the elastic tensile modulus of polypyrrole nanotubes bent in a three-point bending configuration [86]. Later on, in 2005, Wu et al. successfully applied this technique on Au nanowires which is schematically illustrated in Fig. 13(a) showing the loading of a self-suspended nanowire using an AFM-tip [24]. The applied force $F$ is inferred from the deflection $x$ of the AFM cantilever monitored with a quadrant photodiode and the cantilever stiffness $k(F=k x)$. The AFM images displayed in Fig. 13(b) and (c) show a self-suspended $\mathrm{Au}$ nanowire before and after plastic deformation by three-point bending tests. The bending experiments on Au nanowires with diameters ranging from 40 to $250 \mathrm{~nm}$ revealed that the elastic modulus is effectively the same for nanowires and for bulk Au (Fig. 13(d)) while the elastic limit is increased by three orders of magnitude reaching the ultimate limit for gold (Fig. 13(e)).

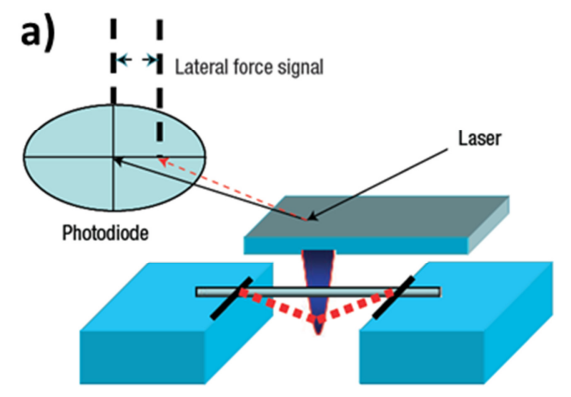

d)

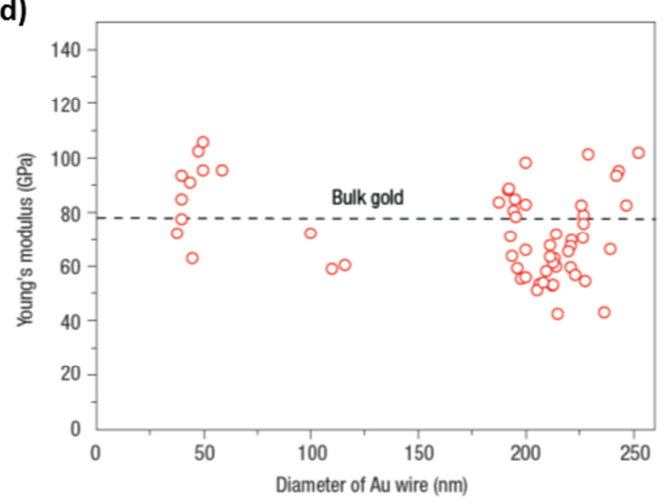

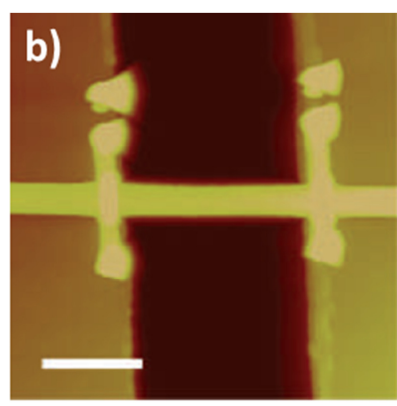

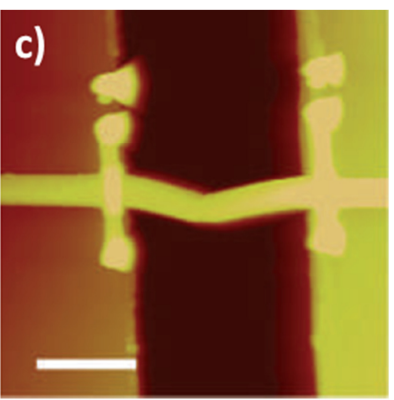

e)

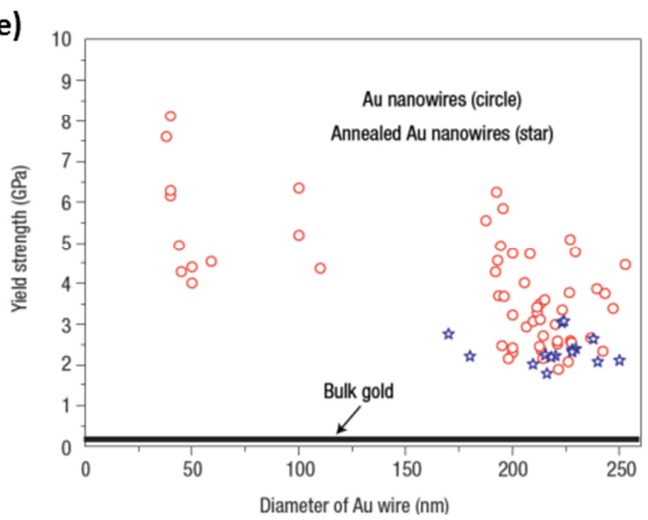

Fig. 13: a) Schematic of a bending test for a self-suspended nanowire using an AFM-tip. AFM image of a Au nanowire with a diameter of $200 \mathrm{~nm}$ b) before and c) after plastic deformation with the AFM-tip. The scale bar is $1 \mu \mathrm{m}$. Measured d) Young's modulus and e) yield strength values for the tested Au nanowires (taken from ref. [24]). 
Note that the nanowires were bent more than their half-thickness making it necessary to take into account higher orders of the beam bending theory which consider the tensile strain developing along the structure for strong deformations. Heidelberg et al. developed a model that describes well the force-displacement curves over the entire elastic range for $\mathrm{Au}$ and Si nanowires [87]. Starting from the well-known beam theory which results in the Euler-Bernoulli equation for slightly bent structures, the authors developed the following equation where the first term corresponds to the Euler-Bernoulli equation for a doubly clamped beam and the second term is a higher order taking into account tensile strain caused by large deformations.

$$
F_{\text {center }}=\frac{192 E I}{L^{3}} \Delta z_{\text {center }}\left(1+\frac{A}{24 I} \Delta z_{\text {center }}^{2}\right)
$$

Here, $E$ is the Young's modulus of the material, $l$ is the moment of inertia, $L$ is the length of the suspended structure, $A$ is the cross-section, $F_{\text {center }}$ is the force applied in the center of the nanowire, and $z_{\text {center }}$ is the deformation in the center of the nanowire.

Recently, McCarthy et al. extended the AFM bending test to measure besides the Young's modulus and the yield strength also the Poisson's ratio $v$ of metal nanowires [88]. For this purpose, the authors developed a four-point electrical measurement on self-suspended nanowires crossing a trench (see Fig. 14). The relative changes of the electrical resistance observed during nanowire bending were analyzed in terms of a model which takes into account bending and tensile deformations. Within the elastic regime, the specific electrical resistivity for metals is assumed not to be affected $(\Delta \rho=0)$, and thus, a fit of equation (7) to the data may be used to determine the value of $v$.

$$
\frac{\Delta \rho}{\rho}=\frac{\Delta R}{R}-2(1+2 v)\left(\frac{\Delta z_{\text {center }}}{L}\right)^{2}
$$




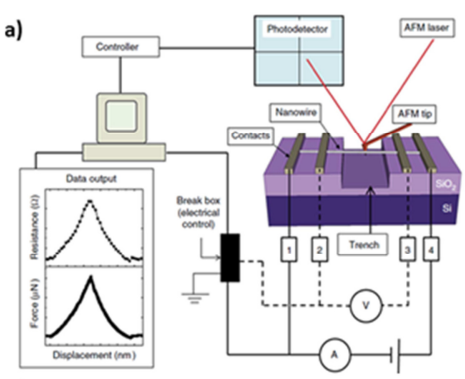

b)

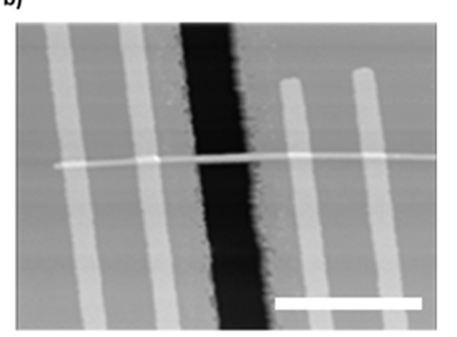

c)

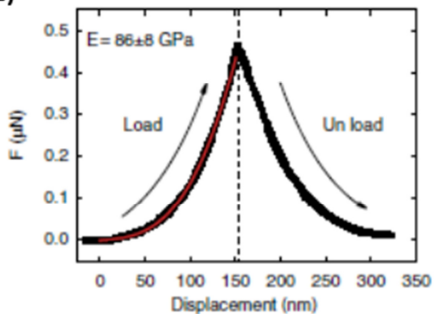

e)

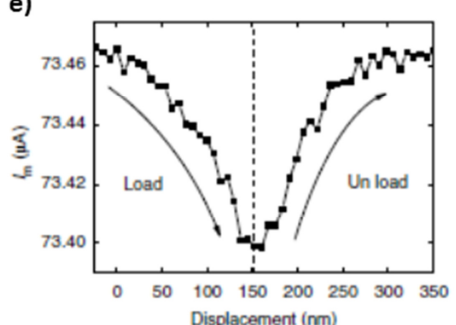

d)

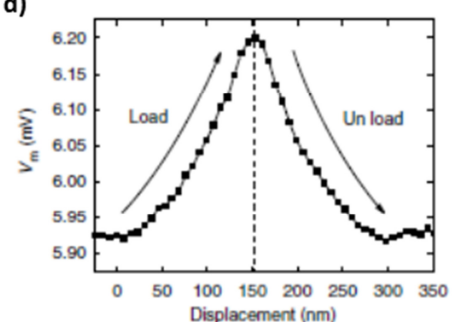

f)

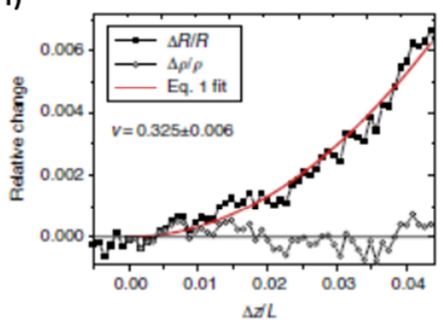

Fig. 14: a) Schematic representation of the nanowire electromechanical experiment. The AFM allows for accurate mechanical characterization by lowering the AFM tip below the axis of the nanowire, into a predefined trench, and laterally driving the tip in the $x-y$ plane perpendicular to the nanowire long axis. The lateral signal from the AFM-tip torsional motion during nanowire loading is measured simultaneously with the four-point resistance giving resistance- and force-displacement curves. b) AFM image of an individual suspended $\mathrm{Ag}$ nanowire electrically contacted at four points (scale bar: $4 \mu \mathrm{m}$ ). c) Applied force, d) measured current, and e) measured voltage as a function of AFM-tip displacement. The red curve in (c) is a fit to the generalized mechanical model [87]. $f$ ) The relative change in resistance and resistivity as a function of $\Delta z / L$ for an individual Ni nanowire with a radius of $40 \mathrm{~nm}$. Taken from ref [88].

A force-displacement curve for an individual Ni nanowire with a radius of $40 \mathrm{~nm}$ is presented Fig. 14(c). The measured voltage and the measured current as a function of the displacement are shown in Fig. 14(d) and (e), respectively. While the voltage increases with increasing displacement, the current diminishes. The measured values of $\Delta R / R$ as a function of $\Delta z / L$ are displayed in Fig. 14(f) revealing a quadratic dependence as predicted by equation (7). The obtained Poisson's ratio $v$ for $\mathrm{Ni}$ nanowires was essentially the same as for bulk $\mathrm{Ni}$. In contrast, for fivefold twinned Ag nanowires $v$ was found to decrease from 0.37 for bulk to 0.225 for the nanowires under study.

\section{I.3.iii Indentation}

As illustrated before, indentation is a comparatively simple experiment giving access to the local mechanical behavior for bulk materials. Nowadays, nano-indentation is routinely employed to study the mechanical properties of thin films as well as for nanostructures. Fig. 15(a) shows a scanning force microscopy image of gold crystals which were fabricated by dewetting a Au thin film on a sapphire substrate. Nano-indentation tests on Au particles with different geometries revealed that the strength of the particles increases with increasing particle size [89], at variance with the "smaller is stronger" usual finding. Molecular dynamic simulations supported the experimental findings and revealed that the decrease of yield strength for smaller particles originates from the emission of 
dislocations at the nanoparticle side facets. It is worth mentioning that such type of nano-indentation may be performed by employing atomic force microscopes simplifying the alignment procedure compared to nano-indenters thanks to the imaging capabilities.
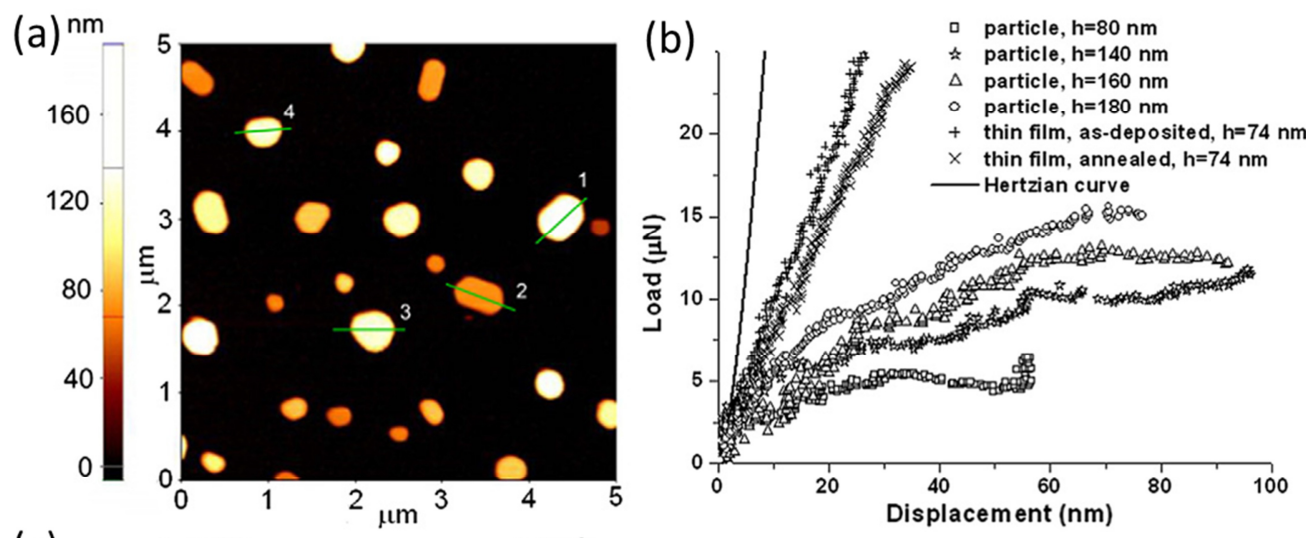

(c)
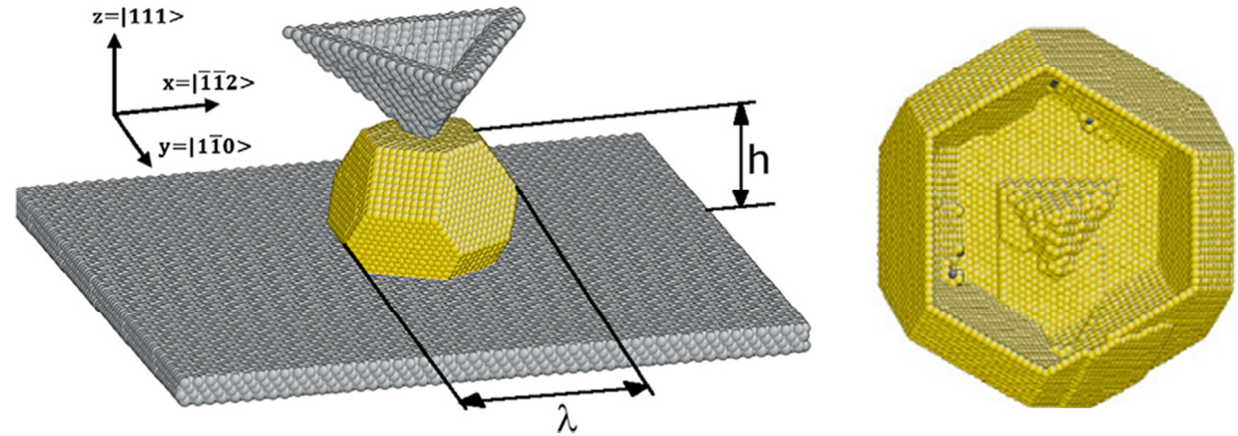

Fig. 15: a) Scanning force microscopy image of Au crystals grown by dewetting a Au thin film on a sapphire substrate. b) Indentation load-displacement curves for particles of different heights. c) Atomic configuration of a molecular dynamics simulation cell for the indentation of a $h=4.9 \mathrm{~nm}$ high Au particle with a Winterbottom shape. d) Au particle after indentation simulated by molecular dynamics [89].

\section{I.3.iv On-chip mechanical tests}

Recently, a novel on-chip nano-mechanical testing laboratory has been developed at UCL (Belgium) [90, 91, 92]. The conceptual design and fabrication process are depicted in Fig. 16(a)-(d). The general idea of this approach is the deposition of the material to be tested on a strained $\mathrm{Si}_{3} \mathrm{~N}_{4}$ film which has been deposited at $800{ }^{\circ} \mathrm{C}$ on top of a sacrificial $\mathrm{SiO}_{2}$ layer. The large internal stress in the $\mathrm{Si}_{3} \mathrm{~N}_{4}$ film is controlled by the deposition conditions. By patterning the films and removing the sacrificial layer beneath, the $\mathrm{Si}_{3} \mathrm{~N}_{4}$ film may relax, thus tensile straining the testing material on top. By varying the length of the $\mathrm{Si}_{3} \mathrm{~N}_{4}$ actuator the stress applied on the testing material may be modified. Lithography facilitates the fabrication of numerous testing structures with different lengths, widths, and shapes on one and the same chip, allowing for studying systematically the mechanical properties and, in particular, the elastic limit of a large number of testing structures. The strain applied on the testing structures is read out by measuring the displacement of cursors, which have been fabricated 
by lithography as illustrated in Fig. 16(c). This method has successfully been applied to Cu, Al, Pd, and Si for measuring their elastic limit and fracture strength.

a)

1. Starting bulk wafer

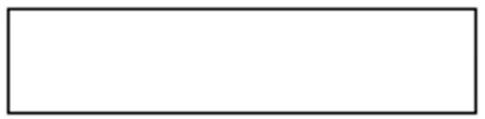

2. Sacrificial layer deposition (around $1 \mu \mathrm{m}$ )

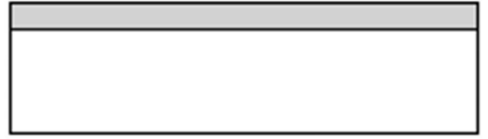

3. Actuator deposition

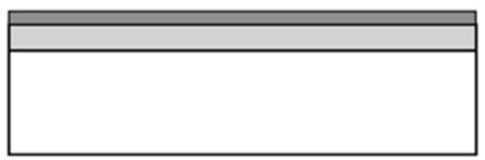

4. Actuator dry etch

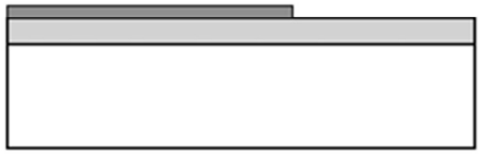

5. Deposition of test material



6. Patterning of test material

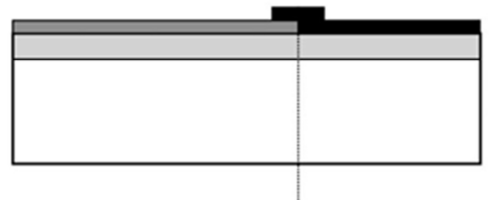

7. Release (sacrificial layer wet or dry etch)

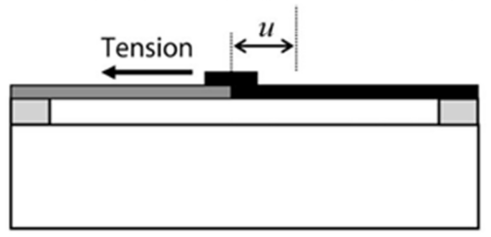

Test material (specimen)

$\square$ Actuator

$\varpi$ Sacrificial layer

$\square$ Silicon substrate b)

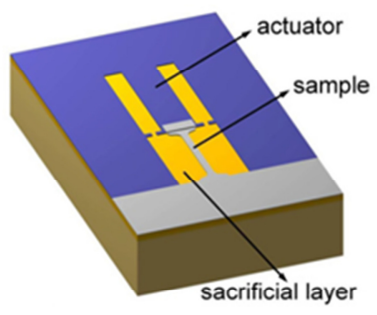

c)

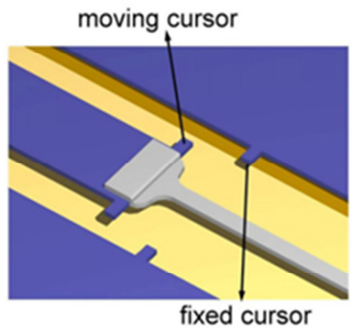

d)



Fig. 16: a) Conceptual design and fabrication process for the lab-on-chip approach [90] where (b) several samples together with their actuator are prepared on one and the same substrate [93]. c) The strain is read-out by the displacement of cursors. d) The variation of the length of the actuator allows for varying the applied strain [93]. 


\section{I.4 In situ micro- and nano-mechanical tests in scanning and transmission electron microscopes}

Within the last decade in situ micro- and nano-mechanical testing apparatuses were developed which allow for visualizing the deformation of the structure and for monitoring the nucleation and evolution of defects in micro- and nanomaterials. Within this sub-chapter recent developments and experimental findings obtained by micro- and nano-mechanical testing within scanning and transmission electron microscopes are reviewed.

As described before FIB milling allows for fabricating dedicated specimen for micro-mechanical testing. Kiener et al. developed a tensile testing stage for in situ mechanical loading in scanning electron microscopes where the microstructures as well as the gripper of the testing stage were machined by FIB milling adapting their forms onto each other (Fig. 17(a) and (b)) [13]. Scanning electron microscopy allows for following the deformation of the microstructure under tension and to correlate events showing up in the stress-strain curve with the appearance of slip traces (Fig. 17(c)(e)).
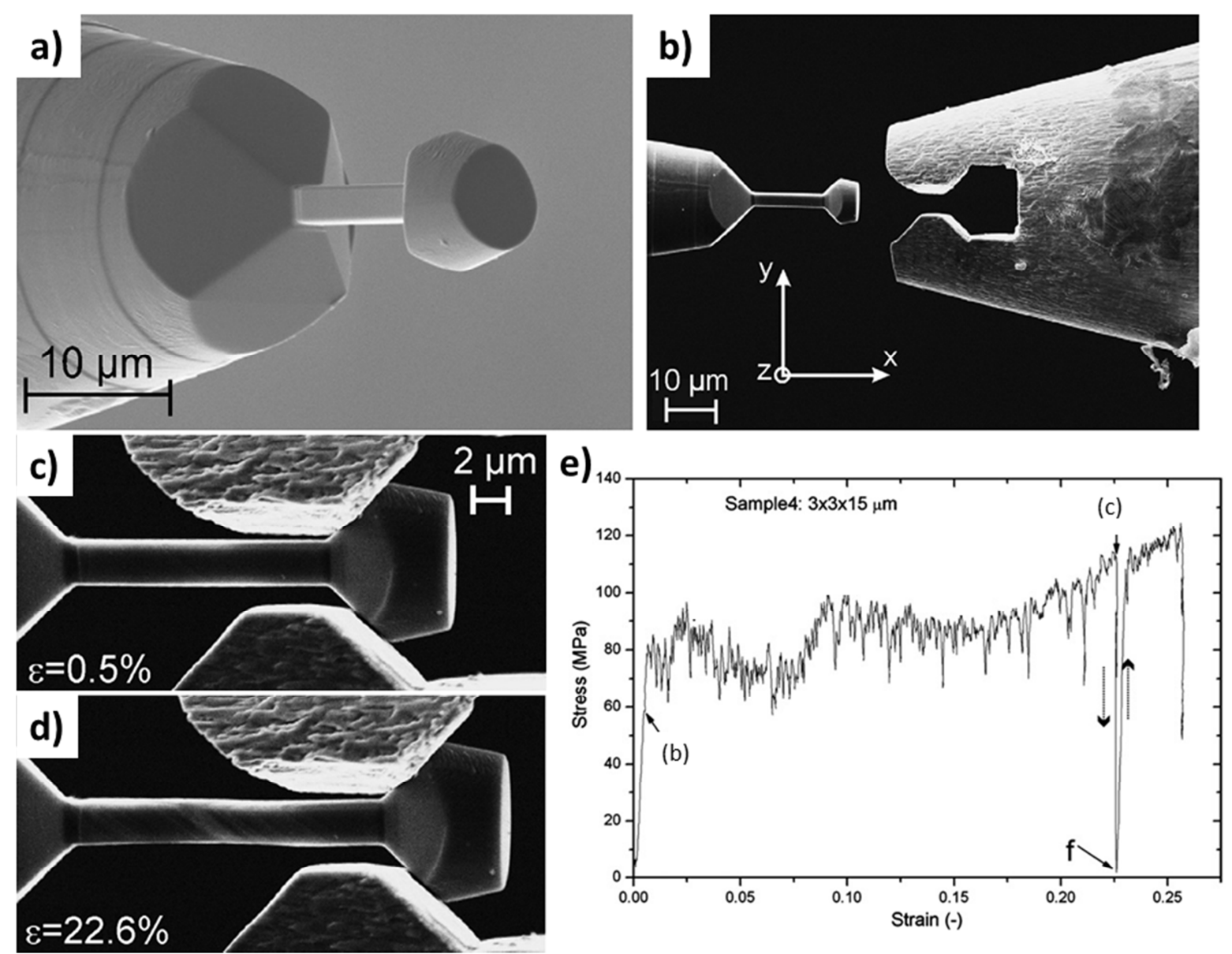

Fig. 17: Scanning electron microscopy image of a) a $\mathrm{Cu}$ tensile testing sample prepared by FIB and b) tungsten sample gripper head. SEM image c) of tensile sample placed in the gripper head and strained by $0.5 \%$ and d) after straining by $22.6 \%$. e) Stress-strain curve where (b) and (c) mark the moment where the respective SEM images were taken [13]. 
Note that SEM is a surface sensitive tool giving access solely to defects which exit at the surface while stored dislocations cannot be investigated. Ex situ Laue microdiffraction experiments on $\mathrm{Cu}$ microstructures which were strained by $22 \%$ evidenced that all dislocations escaped from the strongly deformed crystal while they were stored at the ends of the structure where the sample looks perfect in the SEM [85].

Transmission electron microscopy on the contrary allows for imaging defects and dislocations inside the specimen following their nucleation and propagation. First dynamic observations of plasticity in thin films were realized in the 90s of last century and early 2000s [94, 95]. These first in situ experiments were followed by a series of in situ mechanical studies on various types of films on polymer substrates $[28,96,97]$. The first in situ tensile tests on free-standing sub-micrometric Al single crystals prepared by FIB milling and the observation of dislocation nucleation were demonstrated by S.H. Oh et al. in the late 2000s [98]. While at the beginning dislocation loops are observed, the deformation at later stages is dominated by single ended dislocation sources (Fig. 18(a)-(c)). These studies directly showed the dislocation dynamics evidencing the source truncation in the crystal which implies higher stress for their activation. In addition, while the dislocation nucleation and loss rate were counterbalanced for a given strain rate of $10^{-4} \mathrm{~s}^{-1}$, the increase in strain rate to $10^{-3} \mathrm{~s}^{-1}$ caused a noticeable increase in dislocation density. These findings indicate that the deformation is strain rate sensitive.
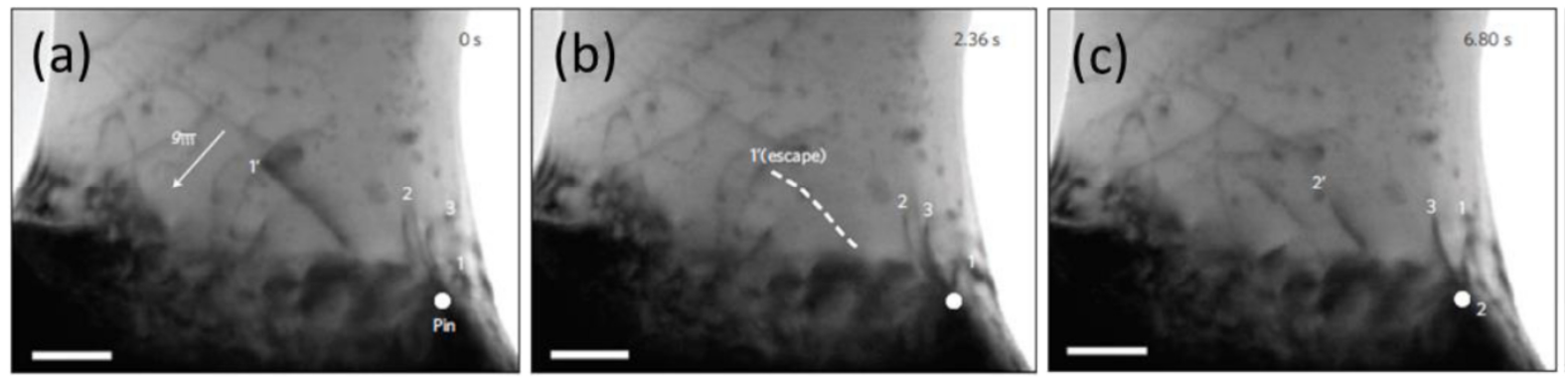

Fig. 18: a) - c) Sequence of in-situ TEM images of an Al single crystal during tensile testing showing single-ended dislocation sources lying on a set of parallel slip planes at $\varepsilon \sim 140 \%$ [98].

In the aforementioned study, the authors reported the escape of dislocations at the surface during deformation. Shan et al. reported the compression of Ni pillars containing a high density of dislocations $\left(\sim 10^{15} \mathrm{~m}^{-2}\right)$ in its pristine state which may have been caused by the FIB milling process (Fig. 19(a)). During compression the dislocations escape at the pillar surfaces resulting in a pillar which is free of dislocations after the mechanical test (Fig. 19(b)) [99]. The corresponding forcedisplacement curve is shown in Fig. 19(c). This phenomenon is termed "mechanical annealing" or "dislocation starvation" which was later on also observed in tension tests [100]. 

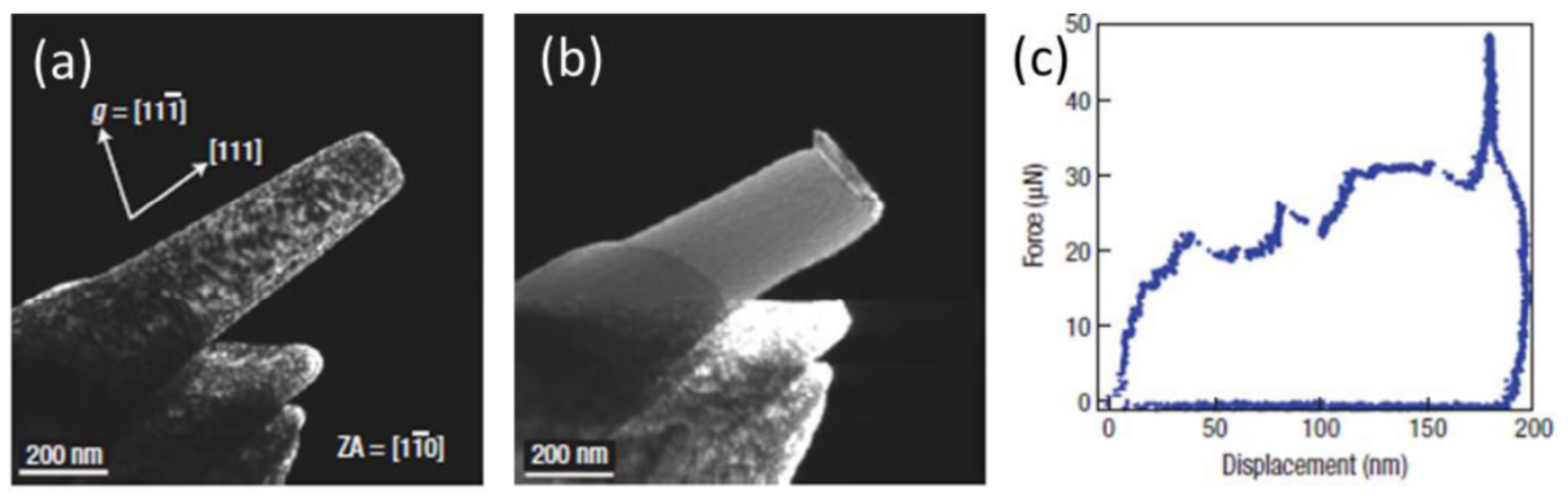

Fig. 19: In situ TEM compression test on a FIB microfabricated 160-nm-top-diameter Ni pillar with $<111>$ orientation. a) Dark-field TEM image of the pillar before the test showing a high initial dislocation density. b) Dark-field TEM image of the same pillar after the test revealing that the pillar is now free of dislocations. Both dark-field images are shown in a $g=[11 \overline{1}]$ condition, zone axis $(\mathrm{ZA})=[1 \overline{1} 0]$. c) Forcedisplacement curve of the compression test [99].

While the aforementioned experiments concentrated on samples that were prepared by FIB milling which may eventually introduce defects and modify the surface state of the fabricated structure, in recent years, in situ testing methods for objects grown by bottom-up approaches were developed. For instance, in situ compression tests on freestanding Au nanostructures inside a SEM revealed that in the absence of mobile defects or cracks, gold can yield unpredictably with apparent energy release rates resulting in pancake-like structures [101]. The nanostructures under study yielded stochastically with values ranging from 16 to $110 \mu \mathrm{N}$ which is fivefold the load necessary for flow after yield.

On the other hand, dedicated miniaturized in situ tensile testing platforms based on microelectromechanical systems (MEMS) were designed by Zhu et al. [102], Han and Saif [103], and Haque and Saif [104] facilitating controlled in situ tensile tests of nanostructures in scanning and transmission electron microscopes as well as in combination with Raman spectroscopy [105]. For eventually testing quasi one-dimensional nano-objects such as nanowires or nanotubes mechanically in electron microscopes, they are harvested individually from their growth substrate using a micromanipulator in a focused ion beam or scanning electron microscope $[106,107]$. Thereafter, the nanowire is glued with one end at the testing stage. Here, the micro-manipulator was employed to actually strain the nanowire. This testing configuration is illustrated in the SEM image in Fig. 20(a) where a $\mathrm{Cu}$ nanowire is fixed at a force sensor and the micro-manipulator. The scanning electron micrographs presented in Fig. 20(b) and (c) show the fracture surfaces of $\mathrm{Cu}$ nanowires after the tensile test revealing shear and brittle failure, respectively [19]. The applied strain during in situ tests in SEMs is typically inferred by digital image correlation (DIC) where the movement of markers deposited on the specimen is compared on the images during the testing and before yielding strain 
resolutions down to $10^{-4}$. These experiments evidenced ultrahigh strengths for the tested nanowires with yield strengths in the range of 1 to $7 \mathrm{GPa}$.

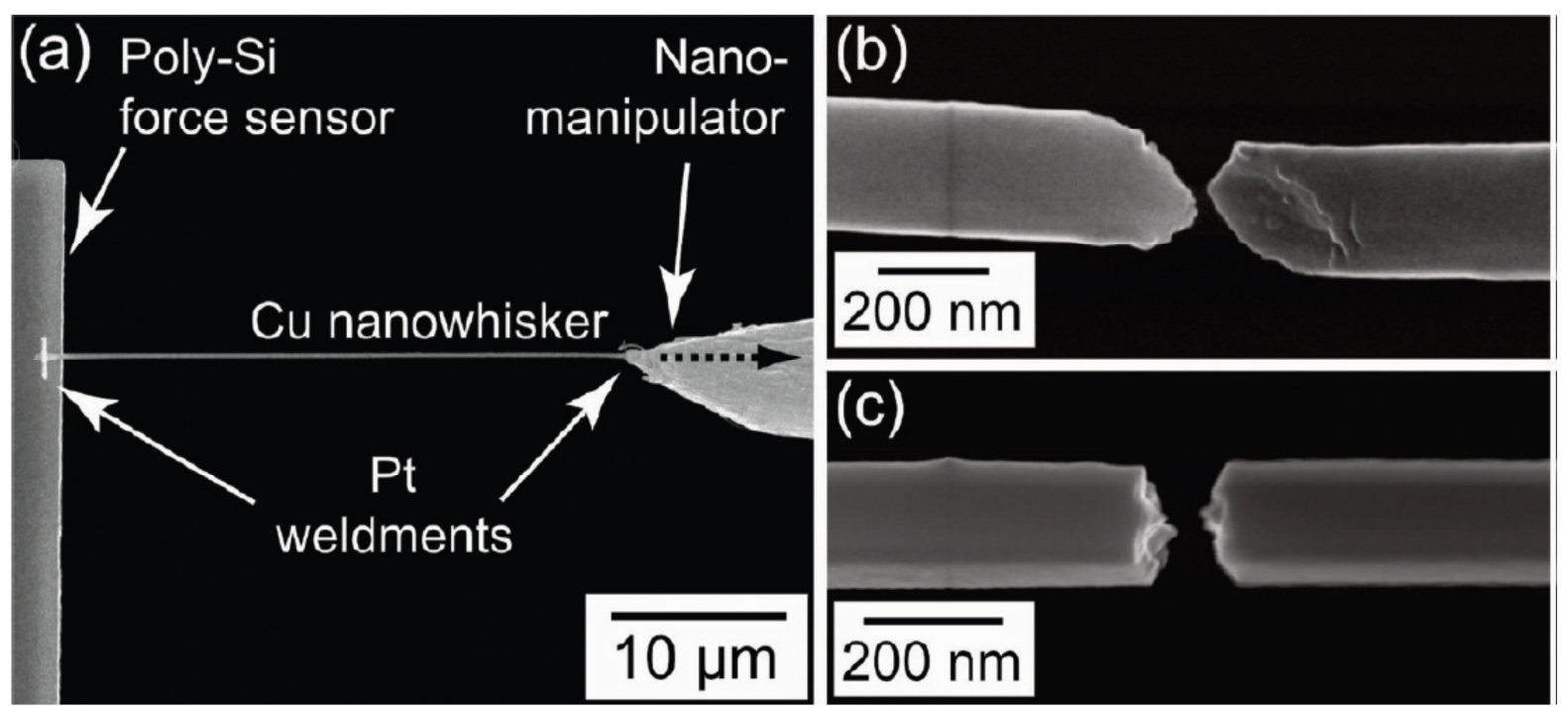

Fig. 20: a) SEM image of a $\mathrm{Cu}$ nanowire mounted between a force sensor and a nano-manipulator for in situ tensile testing. The nanowire is gripped by e-beam deposited Pt weldments, the force applied on the specimen is measured by either a poly-Si flexure beam apparatus or a capacitive-based transducer, and the local strain is measured by digital image correlation of SEM micrographs obtained during testing. SEM images of fracture surfaces of deformed $\mathrm{Cu}$ nanowires showing b) shear and c) brittle failure [19].

Stress-strain curves for Au nanowires investigated by in situ tensile tests in a SEM revealed different mechanical behaviors for nominally the same nanowires [108]. While the first kind shows smooth elastic to plastic transition followed by an abrupt brittle failure (Fig. 21(a)), the second kind shows an immediate brittle failure after the elastic regime (Fig. 21(b)). For both types of wires, the yield strength (ranging from 0.6 to $1.6 \mathrm{GPa}$ ) is much higher than the value reported for bulk gold. It is worth emphasizing that bulk gold is very ductile, which makes this brittle failure very surprising. Post mortem TEM analyses showed twin lamellae in the deformed nanowires. This twin mediated deformation is completely different from the one usually observed in bulk Au (Fig. 21(c)). 

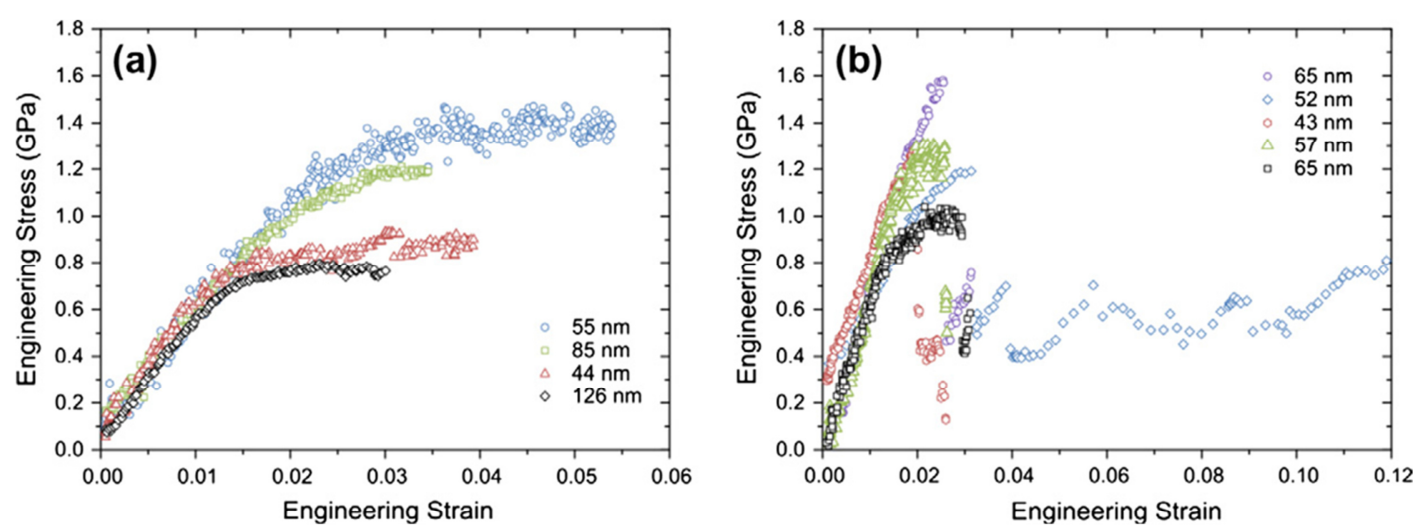

(c)
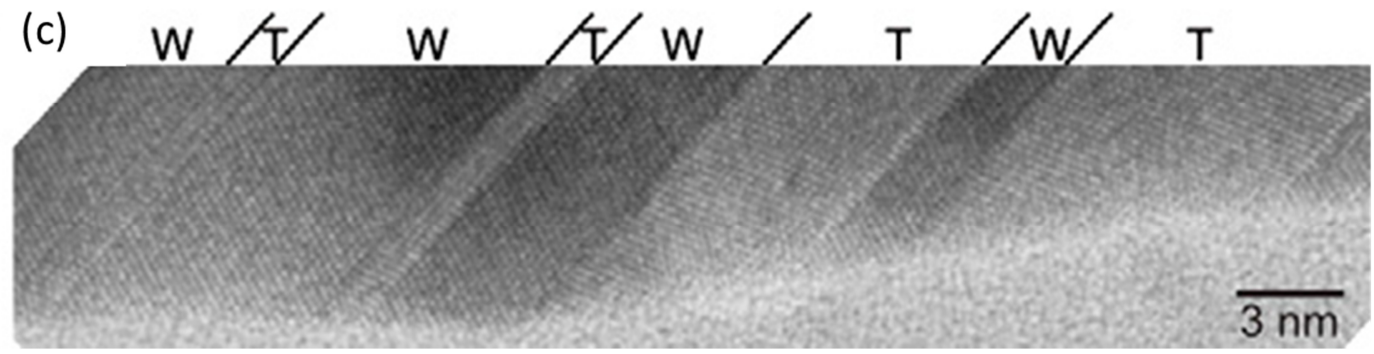

Fig. 21: Stress-strain curves for tensile tested Au nanowires showing a) ductile and b) brittle behavior. c) High resolution TEM image of a deformed nanowire revealing several twinned regions $(T)$ in the matrix of the whisker $(\mathrm{W})[108]$.

Because nano-objects exhibit much higher yield strengths as compared with bulk materials, they can be elastically deformed in a large range, which allows observing non-linear elastic behaviors. Recently, Chen et al. reported anharmonic elastic behavior for tensile strained Pd nanowires [109]. The authors loaded defect-free Pd nanowires using a MEMS based tensile testing device. Fig. 22(a) presents the loading and unloading curves for Pd nanowires with a diameter of 110 and $33 \mathrm{~nm}$. While the loading and unloading curves overlap with each other demonstrating that the deformation was purely elastic the stress-strain curve deviates from a linear behavior for large strain values. This deviation is attributed to higher orders of the elasticity of the material. While the elasticity for low strains can be well described by the interaction of neighboring atoms in a material, for high strains the interaction with the second and third neighbor has to be taken into account resulting in a deviation from the linear behavior of the stress-strain curves.

$$
\varepsilon=\frac{\sigma}{E}+\delta\left(\frac{\sigma}{E}\right)^{2}
$$

Here $\delta$ is a function of the second and third order elastic constants which was found to be 11.2 +/- 3.9 for the $\mathrm{Pd}<110>$ nanowires under study. The higher order elastic constants and thus the anharmonic elastic behavior can be studied on nanomaterials which show elastic limits close to the theoretical values and, thus allow for applying very high strains without entering the plastic regime. 
(a)

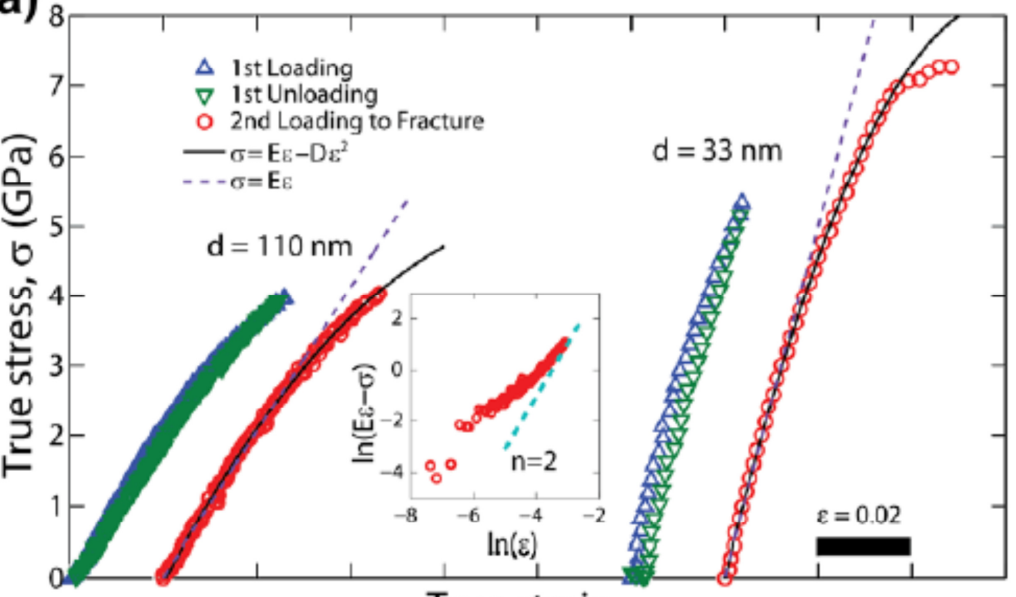

True strain, $\varepsilon$ (b)

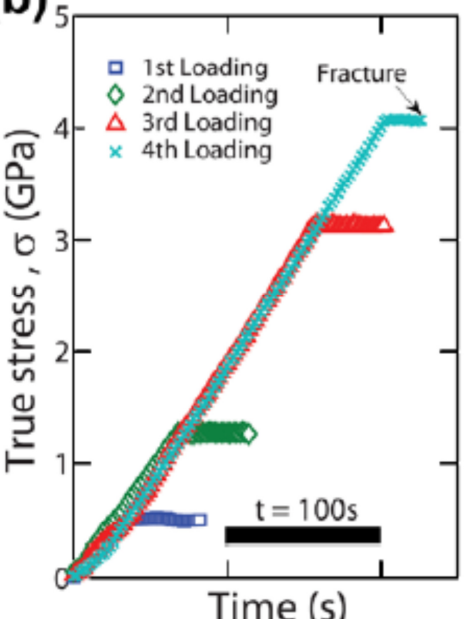

Fig. 22: (a) Loading, unloading, and subsequent fracture stress-strain curves for two Pd nanowires. Linear and quadratic fits (reconciled by the power law exponent shown in the inset) to the fracture curves are shown. b) Stress-time data for a representative Pd nanowire during which the actuator was held stationary, confirming elastic behavior until near fracture stress [109].

By increasing the force range and also varying the temperature Chen et al. was able to investigate the plasticity in single Pd nanowires as a function of temperature giving access to the necessary activation free energy $\Delta G_{\text {act }}$ for nucleating the first dislocation [110]. While typically energies of about $0.4 \mathrm{eV}$ are necessary to nucleate a dislocation, the studies revealed an energy barrier which is ten times lower in the range of $0.03 \mathrm{eV}$. The striking implication of this result is that surface diffusion mediated by stresses is the key ingredient controlling dislocation nucleation at the surface.

Another striking example is the liquid-like behavior recently observed in Ag nanocrystals [111] by in situ high-resolution TEM. Silver nanoparticles were demonstrated to be deformed like liquid droplets while the interior remained highly crystalline with no signs of dislocations. Surface-diffusionmediated pseudoelasticity is evident at room temperature. It can be driven either by an external force or by capillary-energy minimization. This kind of highly unusual Coble-like pseudoelasticity was also evidenced by atomistic simulations to happen for silver particles with sizes smaller than $10 \mathrm{~nm}$ at room temperature and at timescales from seconds to months.

Peng et al. reported on in situ tensile tests of single-crystalline Ni nanowires as a function of the nanowire diameter $d$ and the applied strain rate ranging from $10^{-4}$ to $10^{-2} \mathrm{~s}^{-1}$ [112]. All stress-strain curves are almost linear with minute changes in the slope right before failure and no obvious yielding was detected. The ultimate tensile strength was found to increase with decreasing wire diameter. Additionally, for wires with a similar diameter, the ultimate tensile strength increased with increasing strain rate. Typically, the size dependence of the yield stress is described by a simple power law of $\sigma$ 
$\sim d^{-\alpha}$ analogous to the well-known Hall-Petch relationship. The fitting parameter $\alpha$ in this work amounted to $\sim 0.7$ which is in good agreement with values reported in literature for $\mathrm{Au}$ and Ni pillars. The increase in strain-rate sensitivity is explained by the reduced activation volume in nanowires compared to coarse-grained $\mathrm{Ni}$. The grain refinement from the micro- to the nanoscale can lead to a decrease in activation volume by two orders of magnitude. The work done by ultra-high yield stress on a small activation volume is sufficient to lower the activation energy in order to nucleate dislocations.

While scanning electron microscopy is a surface sensitive tool and, thus it allows for visualizing only the steps arising from dislocations which exit at the sample surface, transmission electron microscopy gives access to the nucleation and evolution of defects within the specimen. Minor et al. demonstrated in situ nano-indentation of aluminum films within a TEM showing the first real time observations of discrete microstructural events such as the formation and propagation of prismatic loops that occur during mechanical deformation [113]. Furthermore, deformation-induced grain growth resulting from grain boundary migration, grain rotation, and grain coalescence was evidenced in ultrafine-grained Al by the same technique [114].

In situ mechanical tests of individual Au nanowires in a TEM demonstrated a reversible cyclic deformation by twinning and de-twinning (Fig. 23) [115]. While during the traction of the Au wire twins were generated, these twins disappeared during the unloading and a subsequent compression. The experimental findings were reproduced by molecular dynamics (MD) simulations. These findings demonstrate the enormous power of in situ compared to ex situ experiments. The twinningdetwinning mechanism can exclusively be studied during the mechanical actuation while the state of the nano-object before and after deformation is the same. 


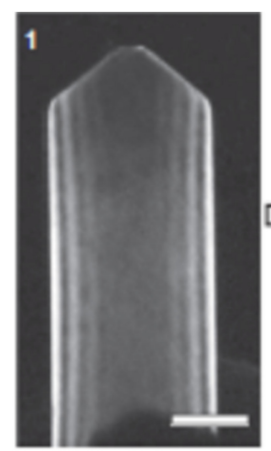

$\varepsilon=0 \%$

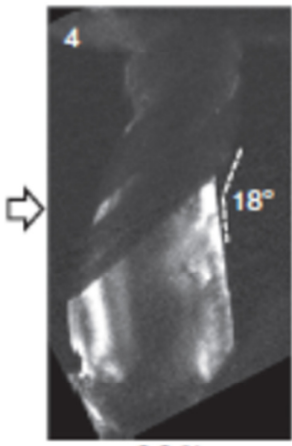

$6.9 \%$

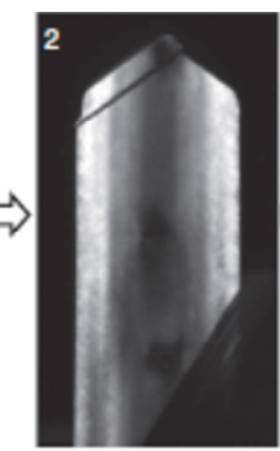

$1 \%$

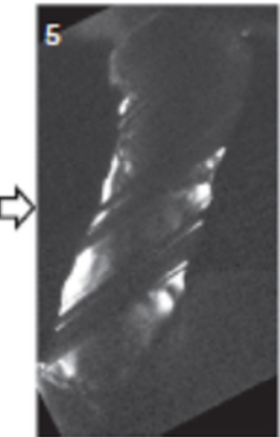

$20.5 \%$



$0 \%$

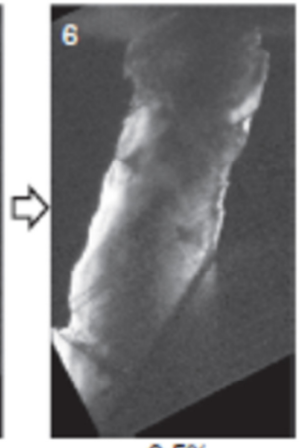

$-0.5 \%$

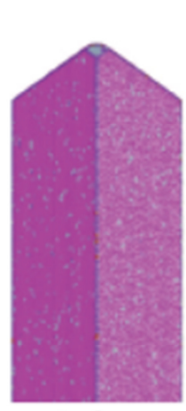

$\mathrm{t}=0 \mathrm{~ns}$
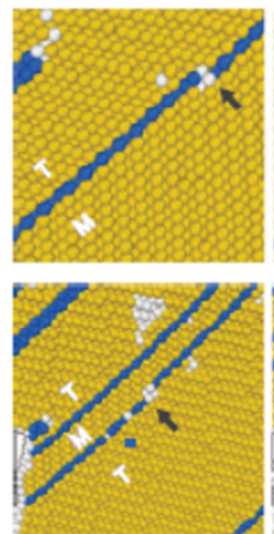

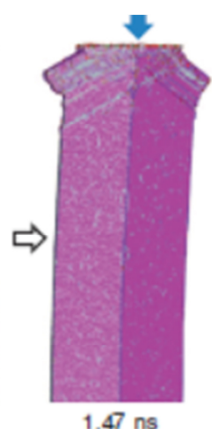

$0.93 \mathrm{~ns}$
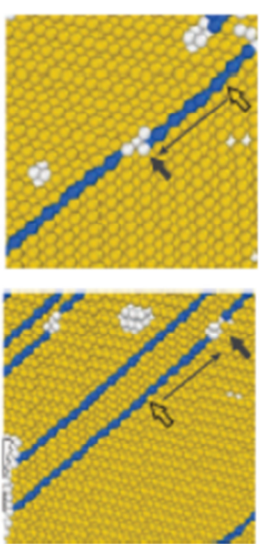

Fig. 23: a) Series of TEM dark-field images captured during cyclic tension-compression. Upon the first tensile loading, a single nanotwin was formed near the tensile grip (image 1 to 2) and then erased completely by detwinning during subsequent compression (image 2 to 3 ). Subsequent tensile deformation was dominated by the successive nucleation and extension of parallel nanotwins along the same slip system (image 4 to 5). As the nanotwins spread over the full gauge length of nanowire while maintaining the same twin variant, the wire axis of the twinned part was rotated by $\sim 18^{\circ}$. A large strain of $\sim 30 \%$ was accommodated by twinning and then recovered by detwinning during subsequent compression (image 6) (scale bar: $100 \mathrm{~nm}$ ). b) Snapshots from MD simulations showing the tensile deformation by twinning and subsequent detwinning of the twinned nanowire upon compressive loading. The colour coding represents the coordination number; magenta: 9 for the $\{111\}$ surface atoms; grey: 8 for the $\{100\}$ surface atoms in the twinned part. c) MD simulation showing the twinning and detwinning processes by the nucleation and glide of partial dislocations. Growth of a twin under tension by successive glide of partial dislocations on the adjacent plane. Detwinning by reverse glide of partial dislocations with the same Burgers vector as the twinning partial dislocations [115].

All the works reviewed above concentrated on FCC metal structures. In the case of semiconductors, Östlund et al. reported a brittle-ductile transition (BDT) for Si nanopillars. While bulk silicon is brittle at ambient conditions and becomes ductile at elevated temperatures, Si nanopillars with a diameter in the range of few hundred nanometers are ductile at room temperature [21]. Similar findings were reported for other semiconductor materials such as GaAs and InSb where the critical diameter for the BDT amounts to about $1 \mu \mathrm{m}$ and $10 \mu \mathrm{m}$, respectively $[20,116]$. However, this BDT was only reported for compression tests while it was not yet found for other nano-mechanical tests such as three-point bending tests on Si nanowires.

Presently, large efforts are undertaken for studying the mechanical properties of micro- and nanoscale materials as a function of temperature as well as a function of strain rate. For instance, 
strain rate dependent tensile tests on silver nanowires revealed a brittle-to-ductile transition. While the Ag nanowires failed brittle for strain rates below $0.02 \mathrm{~s}^{-1}$ they showed ductile behavior for strain rates $>0.2 \mathrm{~s}^{-1}$ [117]. Regarding temperature dependent nano-mechanical tests the biggest challenge is to avoid any temperature gradients and creep. Therefore, sophisticated instrumentation was realized where the samples support as well as the indenter (used for compression tests) is heated simultaneously. The first in situ micro-mechanical testing devices for SEM were performed around 2012 that allow for indentation, compression, tension, and cantilever bending experiments as a function of temperature $[118,119,120]$.

Ex situ and in situ characterization of micro- and nanostructures, either post mortem or during mechanical tests, using scanning and transmission electron microscopy tremendously increased our knowledge about deformation mechanisms governing at small scales. Despite the discoveries that such experiments have provided, these techniques suffer from the fact that imaging or scattering information is either limited to the surface or from a 2D projection of a thin foil of material. In the following chapter, the basic principles of X-ray diffraction will be recapitulated and modern synchrotron X-ray diffraction methods will be introduced which may provide structural information with good spatial resolution and fully in 3D. Thereafter, in situ mechanical tests on micro- and nanostructures using synchrotron X-ray diffraction methods will be reviewed showing their strengths and advantages as well as their limitations. 


\section{X-RAY DifFraCtION}

\section{II.1 Basic principles of X-ray diffraction}

$\mathrm{X}$-rays are electromagnetic radiation which covers a spectrum of wavelengths ranging from 0.01 to $10 \mathrm{~nm}$ corresponding to energies in the range of $100 \mathrm{eV}$ to $100 \mathrm{keV}$. One distinguishes between "soft" and "hard" X-rays depending on their respective energy and, thus, their penetration depth into materials. The separation energy is rather arbitrary. Typically X-rays with energies above $5 \mathrm{keV}$ are regarded as "hard" and below this threshold as "soft". Most X-ray photons impinging on condensed matter are absorbed via the photoelectric process. A very small proportion is scattered elastically by the electrons (Thomson scattering). This very weak interaction is a major advantage since it allows the scattered amplitude to be written as a simple Fourier transform [121, 122, 123] of the electron density:

$$
A(\vec{q})=\int \rho(\vec{r}) e^{i \vec{q} \cdot \vec{r}} d \vec{r}
$$

where $\rho(\vec{r})$ is the electron density in real space and $\vec{q}=\vec{k}_{\text {out }}-\vec{k}_{\text {in }}$ is the scattering vector defined as the difference between the incident wave vector and the scattered one.

This remains an approximation and in the case of perfect crystals it should be carefully justified. Generally, thin crystals with thickness smaller than the extinction length scatter waves in the kinematic regime [124]. In the case of a finite size crystal, considering the Bravais lattice $\vec{R}_{m}$ and the shape function $s(\vec{r})$ one gets:

$$
\begin{aligned}
A(\vec{q}) & =F T\left[s(\vec{r}) \sum_{m} \rho(\vec{r}) * \delta\left(\vec{r}-\vec{R}_{m}\right)\right]=S(\vec{q}) * F(\vec{q}) \sum_{m} e^{i \vec{q} \cdot \vec{R}_{m}} \\
& \propto S(\vec{q}) * F(\vec{q}) \sum_{m} \delta\left(\vec{q}-\vec{G}_{m}\right)
\end{aligned}
$$

where $\rho_{c}(\vec{r})$ is the unit cell electron density, $F(\vec{q})$ its Fourier transform i.e. the structure factor, $S(\vec{q})$ is the Fourier transform of $s(\vec{r}), \vec{G}_{m}$ are reciprocal space vectors, $\delta$ is the Dirac distribution, and the asterisk * represents the convolution product. This very well-known expression simply shows that the diffraction pattern of an unstrained crystal consists in well-defined Bragg peaks at positions given by the Laue condition: $\vec{q}=\vec{G}_{m}$.

A convenient way for visualizing Laue diffraction conditions is the Ewald sphere which is depicted in Fig. 24 [125]. Its construction allows the determination of which lattice planes - 
represented by the reciprocal lattice points - result in a diffraction signal for a given wavelength $\lambda$ of incident radiation. Its radius is given by the modulus of the incident wave vector $\left|\vec{k}_{i n}\right|$. For elastic scattering both the incident and the diffracted wave vectors have the same length $\left|\vec{k}_{\text {in }}\right|=\left|\vec{k}_{\text {out }}\right|$. Hence, for monochromatic radiation, the reciprocal lattice points, which may result in a diffraction signal must lie on the Ewald sphere.

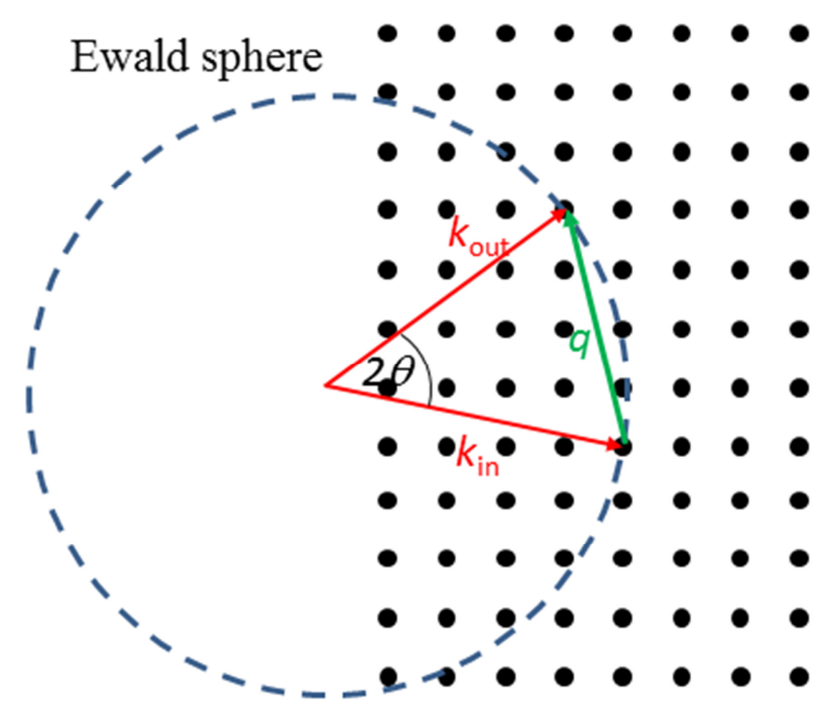

Fig. 24: Schematic of the Ewald sphere whose radius is given by the length of the incident wave vector $\left|\vec{k}_{i n}\right|$. The diffraction points, which may result in a diffraction signal must lie on the Ewald sphere.

As may be seen from formula (10) the Fourier transform $S(\vec{q})$ of the crystal shape is transferred by convolution on any reciprocal space node. As a general rule, one may note that extended objects in real space are small in reciprocal space while small objects in real space result in extended diffraction signals in reciprocal space. Therefore, an infinite three-dimensional crystal of periodically arranged atoms results in periodically arranged sharp Bragg reflections. In the case of a semi-infinite object, the introduced surface can be regarded as an infinite two-dimensional plane with a finite thickness of a few atomic layers in the third dimension. This confined thickness in real space leads to an extended reflection in reciprocal space - the crystal truncation rod (CTR). In the mathematical description, the Fourier transformation of an infinite two-dimensional plane results in a onedimensional line standing perpendicular on the plane - the CTR. This crystal truncation rod connects the Bragg reflections (see Fig. 25(a)) and it is the weakest in the center between two reciprocal lattice points. Studies of the CTR intensity give access to the surface morphology [126, 127].

Considering a finite object which may be a single grain in case of polycrystalline material or a nanosized monocrystal, the shape of the structure affects the diffraction pattern. Each surface creates a crystal truncation rod. Thus, for instance, for a hexagonal object (Fig. 25(b)) the shape 
factor results in a six-fold symmetrical diffraction pattern (see (Fig. 25(c)). This shape factor is independent of the scattering vector and, hence, it appears at every Bragg reflection (see (Fig. 25(d)). In addition, the finite size of the structure results in diffraction fringes related to the object size.

a)

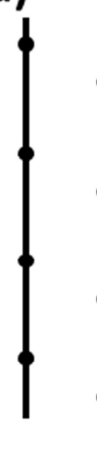

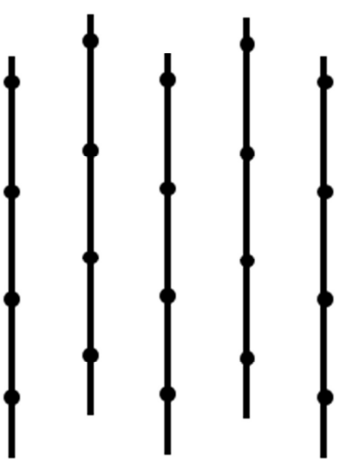

b)

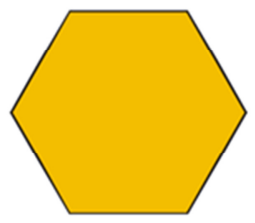

c)

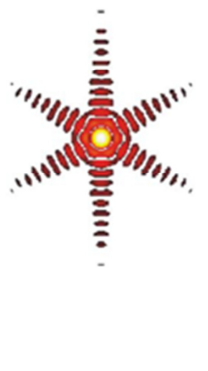

d)

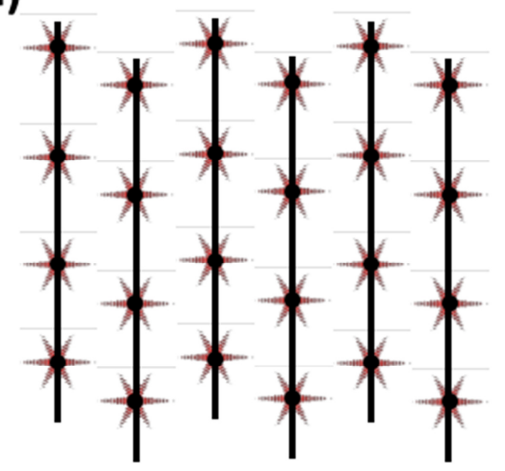

Fig. 25: a) Schematic of the crystal truncation rods (CTRs) originating from the sample surface connecting the Bragg reflections. b) Schematic of a hexagonal object whose shape factor results in c) a sixfold symmetrical Bragg reflection which d) appears at every reciprocal lattice point.

In practice, it is not the amplitude $A(q)$ that is measured but the intensity $I(q)=|A(q)|^{2}$. In the case of an assembly of crystals illuminated by an incoherent beam, one will measure an incoherent addition of intensities on the detector and, since assembly of objects is seldom mono-disperse, generally blurred patterns are obtained with loss of information. One is then left with statistical information from size and shape that may be extracted from line profiles. On the other hand, if a single object is illuminated by a coherent beam with coherence lengths larger than the object size, one obtains a coherent diffraction pattern, which contains all the information on the object. This is what is generally called coherent diffraction. Here, the coherence of the beam means that all points of the wavefront impinging on the sample are correlated. Coherent diffraction from a single $\mathrm{Au}$ crystal [128], $1 \mu \mathrm{m}$ in size, has been reported. The corresponding diffraction pattern show distinct fringes and streaks, which are clear indications that one is indeed dealing with $|S(\vec{q})|^{2}$. Inverse Fourier transforming such data yields the Patterson function, which is the autocorrelation function of the density. However, the crystal shape determined from the diffraction data remains ambiguous due to the fact that the phase of the scattered amplitude is actually not recorded. This so-called phase problem can be solved using phase retrieval algorithms. This technique called Coherent Diffraction Imaging (CDI) will be presented and discussed in section II.4.

Considering a strained crystal (with restricting ourselves to purely elastic strains) and taking into account both that elasticity describes the distortion of the crystalline lattice [129] (and not of the basis) and that elastic strains are always small (of the order of few \% even in nanosized crystals), the structure factor $F(\vec{q})$ can be presumed to be not affected. The crystalline lattice is thus distorted: 


$$
\overrightarrow{R^{\prime}}=\vec{R}+\vec{u}
$$

where $\vec{u}$ is the displacement field. Diffraction is sensitive to the displacement and not simply to strain, i.e. diffraction is also highly sensitive to lattice rotation. If $i=1,2,3$ are the principal orthogonal directions in space, the strain and rotation tensors [130] $\varepsilon_{i j}$ and $\omega_{i j}$ write

$$
\varepsilon_{i j}=\frac{1}{2}\left(\frac{\partial u_{i}}{\partial x_{j}}+\frac{\partial u_{j}}{\partial x_{i}}\right) \text { and } \omega_{i j}=\frac{1}{2}\left(\frac{\partial u_{i}}{\partial x_{j}}-\frac{\partial u_{j}}{\partial x_{i}}\right)
$$

within the framework of the small displacements approximation. The amplitude scattered from the strained crystal writes now

$$
A(\vec{q})=S(\vec{q}) * F(\vec{q}) \sum_{m} e^{i \vec{q}\left(\vec{R}_{m}+\vec{u}_{m}\right)}
$$

In the vicinity of Bragg peak $\overrightarrow{\mathrm{G}}_{0}$, one may write $\overrightarrow{\mathrm{q}}=\overrightarrow{\mathrm{G}}_{0}+\overrightarrow{\mathrm{g}}$ and neglect the term $\overrightarrow{\mathrm{g}} \cdot \overrightarrow{\mathrm{u}}$. Thus:

$$
A(\vec{q}) \approx S(\vec{q}) * F(\vec{q}) \sum e^{i \vec{G}_{0} \vec{u}_{m}} e^{i \vec{q} \vec{R}_{m}}
$$

This shows that to a very good approximation (13) the amplitude scattered by the deformed crystal may be described as the Fourier transform of a modified electron density:

$$
A(\vec{q}) \approx F T\left\lfloor\rho(\vec{r}) e^{i \vec{G}_{0} \vec{u}(\vec{r})}\right\rfloor
$$

Consequently, a strained crystal behaves as if it had a complex electron density with a phase factor $\vec{G}_{0} \cdot \vec{u}(\vec{r})$. This phase strongly influences the intensity distribution and may be determined via phase retrieved coherent diffraction patterns. At variance with the size effect previously described, this strain effect is clearly Bragg peak-dependent. When the strain is homogeneous throughout the crystal under investigation, the displacement field varies linearly with position and the amplitude remains unaffected. The strain is then deduced directly from the shift in peak position. This forms the basis of many methods to determine strains and stresses in materials. Aside from the shift of the mean Bragg position coherent diffraction patterns contain detailed information on the size and the strain field within the crystal (see Fig. 26(i)). In the case of incoherent scattering Bragg peak 
broadening may be used to deduce the size of the diffracting crystal and/or the inhomogeneous strain (see Fig. 26(ii)). In the latter case the peak broadening increases with the distance from the origin of reciprocal space whereas size broadening is independent from the position in reciprocal space. This dependence is used to separate strain broadening from size broadening.

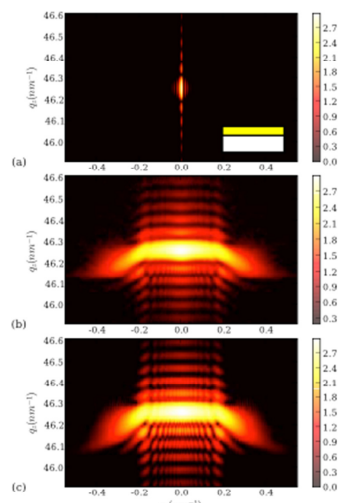

(i)

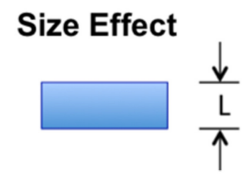

Real space

Strain Effect

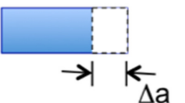

$\Delta Q=-|Q| \Delta a / a$

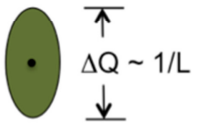

Reciprocal space

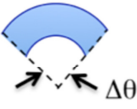

$\Delta Q=|Q| \Delta \theta$

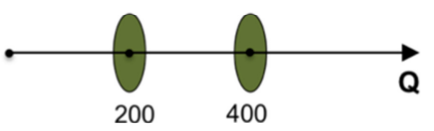

$\Delta Q=$ independent of $|Q|$



$\Delta \mathrm{Q} \sim \mathrm{QQ}$

(ii)

Fig. 26: (i) a) Simulated coherent diffraction pattern for an unstrained Si line. The fringes along the vertical direction arise solely from the size. b) Coherent diffraction pattern from a Si line strained by a nitride cap showing the same size fringes in the vertical direction as for the unstrained case. The broadening in the horizontal direction is dominated by the strain gradient. c) Full simulation taking into account the strain field shows good agreement with experimental data shown in (b) (after [131]). (ii) Schematic of size and strain broadening of diffraction peaks. 


\section{II.2 Focusing optics}

In standard laboratory diffractometers, the size of the X-ray beam is typically in the range of few millimeters. Such broad X-ray beams allow for studying an ensemble of nanostructures but they do not give access to the properties of one single nano-object. In order to examine individual nanostructures the size of the X-ray beam has to be in the same order as the size of the object, i.e. the X-rays have to be focused down to the $100-\mathrm{nm}$ regime.

The refractive index for electromagnetic waves is described by the following equation

$$
n=1-\delta-i \beta
$$

Note that the refractive index for X-rays in matter is $<1$ which may lead to total reflection. The critical angle of reflection is defined by the real part $1-\delta$ which scales quadratic with the atomic number $\left(1-\delta \propto Z^{2}\right)$ while the imaginary part $\beta$ that defines the absorption raises with the fourth power $\left(Z^{4}\right)$. Typical values for $\delta$ and $\beta$ are in the range of $10^{-5}$ and $10^{-7}$, respectively. Thus, the refractive index for hard $\mathrm{X}$-rays is very close to unity. This fact complicated the development of focusing optics for hard X-rays. Recent progresses in the fabrication of diffractive, refractive, and also reflective $X$-ray optics applied at $3^{\text {rd }}$ generation synchrotron radiation sources gave rise to tremendous advances in micro- and nanofocusing.

Note that the synchrotron X-ray source, meaning the electron beam, is not a point source but it is extended. Therefore, focusing optics only allow for a demagnification of the source size which is typically in the range of several tens of micrometers. Nowadays, the length of a typical synchrotron undulator beamline is around $50 \mathrm{~m}$ and the distance of the focusing optics from the sample varies between few centimeters up to the meter range. Thus, demagnification ratios ranging from several tens to one thousand are obtained resulting in focal sizes in the range of few hundreds of nanometers. While focusing optics installed very close to the sample position offer very small foci, they limit the possibility of installing special sample environments such as heating stages, cryostats etc. Currently, many synchrotron beamlines are extended in length to $>100 \mathrm{~m}$ opening the possibility to further reduce the focal size, or for keeping the same focus as before for optics with larger working distances and, thus, for allowing the installation of sample environments. The smallest focal spot obtained at the $1 \mathrm{~km}$ long beamline BL29XUL of SPRING-8 amounted to $7 \mathrm{~nm}$ in vertical direction [132]. Besides the advantages concerning the focal size, the elongation of beamlines also increases the lateral coherence length of the X-ray beam which is important for diffraction studies using coherent beams as will be shown in section II.4 [133, 134, 135, 136]. 


\section{II.2.i Reflective Optics}

Conventional mirrors, as used for visible light, at normal incidence cannot be used for hard Xrays, because the reflectivity is too low. A high reflectivity is obtained for grazing incidence when the incident angle is smaller than the critical angle of reflection which is defined by the real part of the refractive index

$$
\begin{aligned}
& 1-\delta=\cos \theta_{c} \\
& \theta_{c} \approx \sqrt{2 \delta}
\end{aligned}
$$

Taking into account $\delta^{\sim} 10^{-5}$, the critical angle $\theta_{c}$ is typically of the order of few milliradians and it increases with increasing $Z$. To reduce the astigmatism Kirkpatrick and Baez proposed the use of two elliptical mirrors in a crossed configuration [137]. Fig. 27 shows such a geometrical arrangement which became known as Kirkpatrick-Baez (KB) mirrors. A major advantage of mirrors as focusing optics is their achromaticity, i.e., the focal length is independent of the energy and respective wavelength of the incident radiation. Thus, they are used for focusing monochromatic beams as well as white beams. In addition, they allow for varying the energy of the incident X-ray beam which is mandatory for absorption and spectroscopy studies.
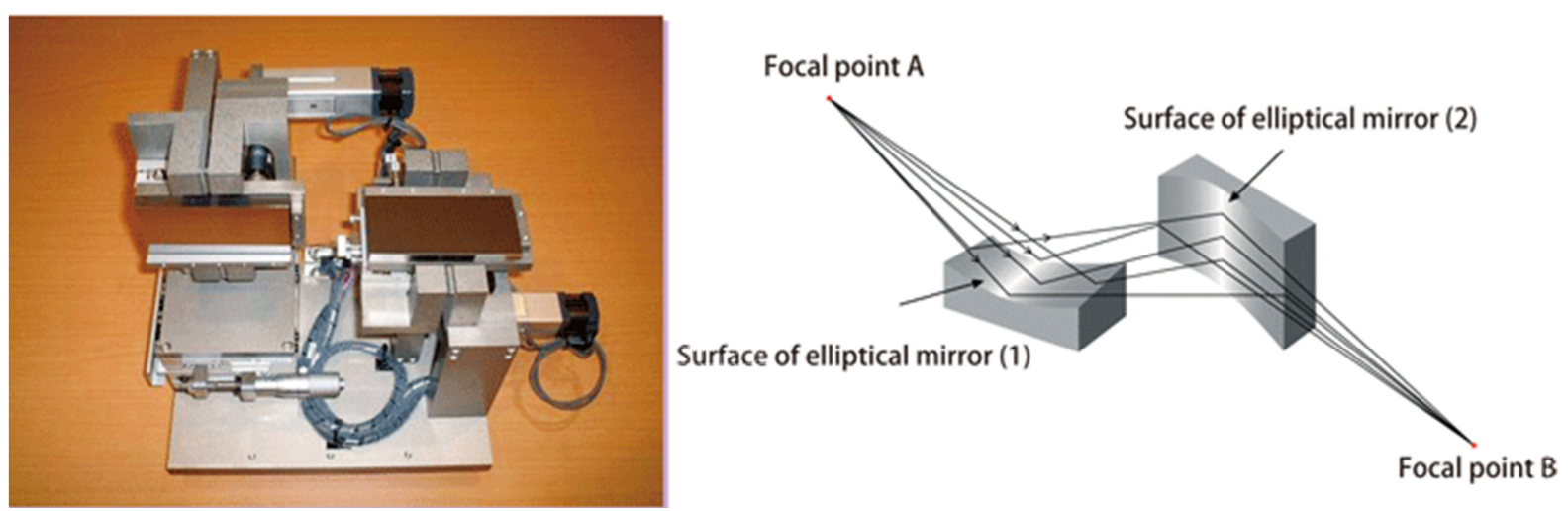

Fig. 27: a) Picture and b) schematic view of a Kirkpatrick-Baez system consisting of elliptical two mirrors which focus an incoming X-ray beam the horizontal and the vertical direction (taken from [138]).

\section{II.2.ii Refractive Optics}

Refractive lenses made of glass are among the most widely used optical components for visible light. For a long time, refractive lenses were considered unfeasible for $\mathrm{X}$-rays due to the weak refraction and strong absorption. However, in the 90s of the last century Snigirev et al. demonstrated that hard X-rays can be focused by concave lenses (Fig. 28(a)) $[139,140]$. The concave shape results from the fact that the refractive index of hard X-rays is smaller than unity. The focal length $f$ which is defined by the equation below is only positive for lenses exhibiting a negative radius $R$, i.e., having a concave shape. 


$$
\frac{1}{f}=\frac{2(n-1)}{R}
$$

Thus, for a biconcave lens with a parabolic profile $x^{2}=2 R y$ as depicted in Fig. 28, the focal length is given by the following equation.

$$
f=R / 2 \delta
$$

a)

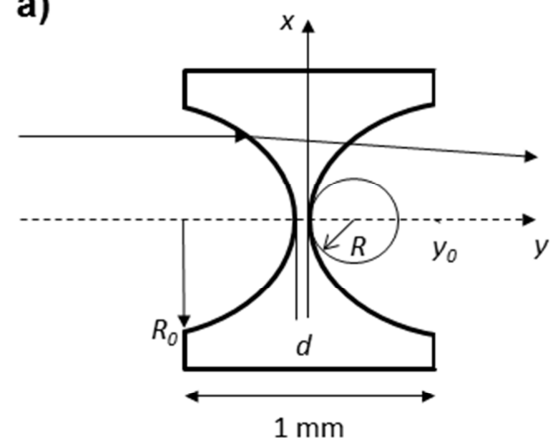

c)

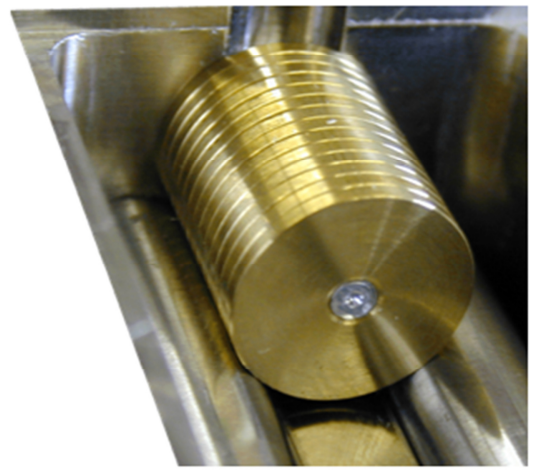

b)
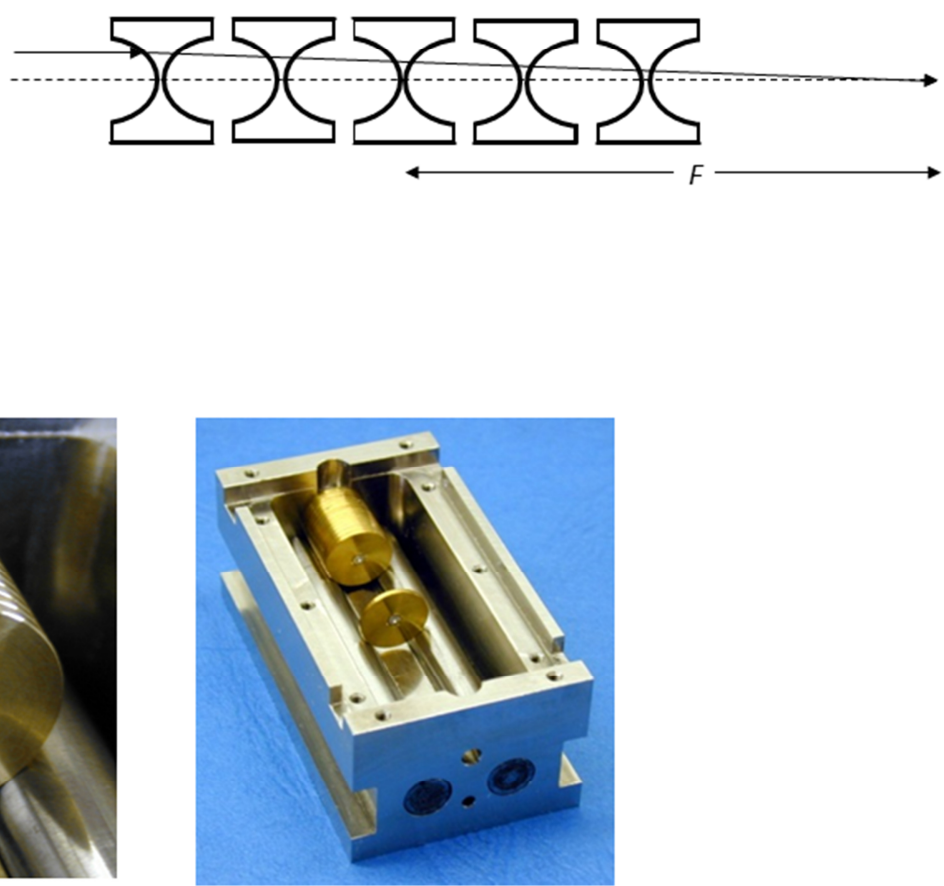

Fig. 28: Schematics a) of a biconcave lens with radius $R$ and thickness $2 w_{0}+d$ and b) the arrangement of several lenses as compound refractive lens with focal length $F$. c) Photographs of an assembly of compound refractive lenses [141].

For a typical radius of curvature of $200 \mu \mathrm{m}$ and considering the value for $\delta$ being in the range of $10^{-5}$, the focal length is in the range of several tens to one hundred meters. In order to achieve a focal length of the order of $1 \mathrm{~m}$, several lenses have to be stacked behind each other (Fig. 28(b)). This arrangement, which is illustrated by the photographs in Fig. 28(c) is known as compound refractive lens (CRL). To minimize absorption and due to the fact that the absorption rises with $Z^{A}$, the lenses have to be prepared out of light materials such as Be, B, C, Al, and Si. The aperture for a lens with a thickness $2 y_{0}+d$ amounts to $2 R_{0}=2 \sqrt{2 R y_{0}}$. 


\section{II.2.iii Diffractive Optics}

Diffraction refers to phenomena which occur when waves encounter obstacles. When light passes through a double slit, a circular wave emanates from each slit which interfere with each other. This experiment was first performed by Thomas Young in the year 1803. For a grating with an arbitrary number of slits the interference fringes are described by the following equation

$$
m \lambda=d \cdot \sin \theta_{m}
$$

where $m$ is the order of diffraction, $d$ is the inter-slit distance, and $\theta_{\mathrm{m}}$ is the diffraction angle of the diffraction order $m$.

Diffraction gratings do not have to consist necessarily of straight slits. For instance, a Fresnel zone plate (FZP) is a circular diffraction grating. Its focusing properties were first discussed in the late $19^{\text {th }}$ century, and Baez originally suggested its use as X-ray optical element. A FZP consists of a series of $N$ concentric rings with a radius of $r_{N}^{2}=N \lambda F$ (see Fig. 29 [142]). The width $\mathrm{d} r_{\mathrm{n}}$ of the rings decreases with increasing radius. The focusing capability is based on constructive interference of the wavefront modified by the passage through the zone plate. Such FZPs are conventionally fabricated by electron beam lithography or by holographic techniques $[143,144]$.

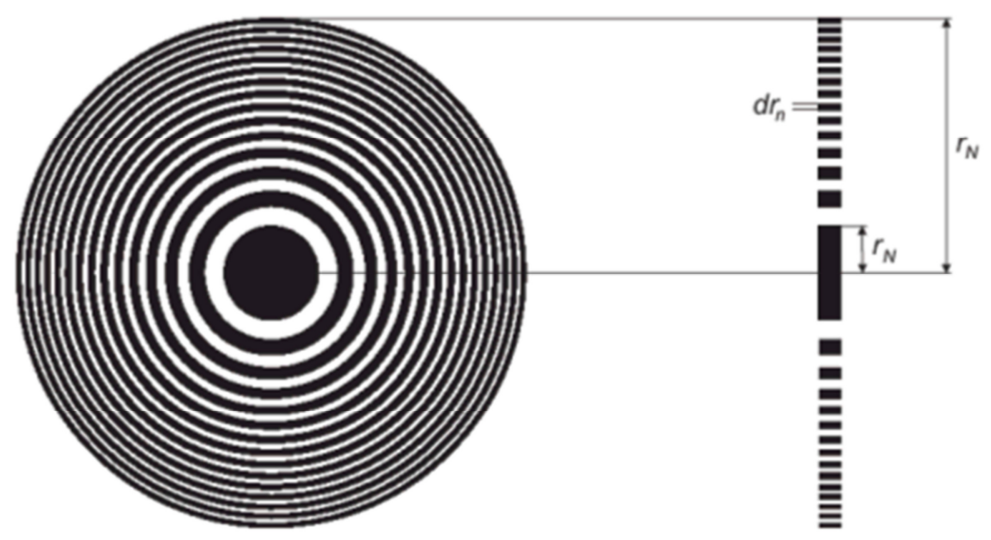

Fig. 29: Schematic of a Fresnel zone plate with $N$ rings and a radius $r_{N}$. The width of the $n$-th $\operatorname{ring}$ is $d r_{n}$. (taken from [142]).

Besides the first order of diffraction higher diffraction orders $m$ exist as well as the $0^{\text {th }}$ order. These additional diffraction orders deteriorate the first order beam focus and, hence, they have to be eliminated. For this purpose, a pinhole - the order sorting aperture (OSA) - is introduced as close as possible to the focal point of the first order. However, the OSA still allows the $0^{\text {th }}$ order to penetrate and, thus a central beam stop (CBS) which has at least the same size as the OSA, is installed right in front of the Fresnel zone plate. The complete setup with a Fresnel zone plate is schematically illustrated in Fig. 30. 


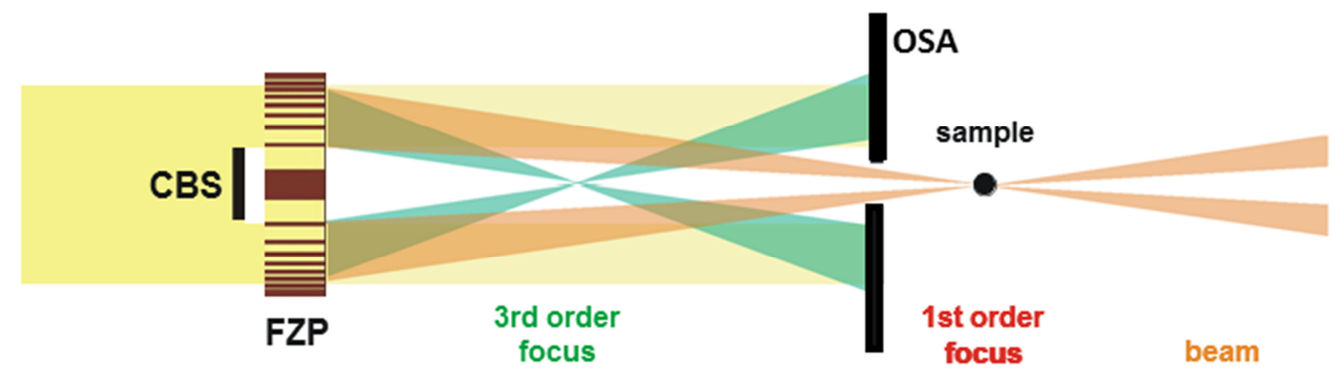

Fig. 30: Schematic of a Fresnel zone plate (FZP) setup which consists of the FZP itself, an order sorting aperture (OSA) for selecting one particular diffraction order of the FZP (here the $1^{\text {st }}$ order of diffraction), and a central beam stop $(C B S)$ to absorb the $0^{\text {th }}$ diffraction order.

Just as the Rayleigh resolution of a light microscope lens is determined by its numerical aperture (NA) the Rayleigh resolution of a zone plate is determined by its maximum diffraction angle $N A=\lambda / 2 d r_{n}$, so that the Rayleigh resolution is $1.22 d r_{n} / m$. Typical values for diffraction limited resolution at an X-ray energy of about $10 \mathrm{keV}$ and with a focal distance of $10 \mathrm{~cm}$ by lithographically produced FZPs are below $100 \mathrm{~nm}$. The Fresnel zone plate is a chromatic focusing device and, thus, the focal length $f=D \cdot d r_{n} /(m \lambda)$ as well as the depth of focus $D O F= \pm 4 d r_{n} /(m \lambda)$ depends on the wavelength (or energy) of the incident X-rays. At an energy of $10 \mathrm{keV}$, the focal length and the DOF of the FZP (with an outer zone width of $d r_{\mathrm{n}}=70 \mathrm{~nm}$ and a diameter of $D=200 \mu \mathrm{m}$ ) used at beamline ID01 at the European Synchrotron ESRF in Grenoble amount to $112.9 \mathrm{~mm}$ and $\pm 160 \mu \mathrm{m}$, respectively.

\section{II.2.iv Advantages/Disadvantages of different focusing optics}

The three different types of focusing optics have their respective advantages and disadvantages. Mirrors (section II.2.i) are achromatic devices and, thus, they allow focusing monochromatic radiation as well as white beams. Hence, they facilitate energy changes necessary for X-ray absorption spectroscopy or 3D X-ray diffraction by the energy tuning approach. In contrast, refractive (section II.2.ii) and diffractive (section II.2.iii) optics are chromatic with a bandwidth of about $\triangle E / E \sim$ $10^{-3}$. The chromaticity, i.e., the dependence of the focal distance as a function of the energy is more pronounced for refractive lenses than for diffractive gratings. The focal length is directly proportional to the energy for Fresnel zone plates while it rises with $E^{2}$ for refractive lenses. Concerning the preservation of the coherence of the beam, diffractive gratings are very well suited since they do not alter the wavefront of the incident beam while the asperity of mirror surfaces and refractive lenses may disturb the wavefront. Major advances have been made within the last years concerning the polishing of mirror surfaces rendering possible the fabrication of surfaces with an rms of the height distribution well below $1 \AA$ over a mirror length of $1 \mathrm{~m}$ and, thus, minimizing the wavefront 
perturbation and even yielding an increased coherent flux compared to other optics. Besides the influence of the focusing optics on the beam properties they may also limit the space available to install specific sample environments for in situ studies. While the distance between all focusing optics and the sample position is the same in order to obtain the same focal spot size, the order sorting aperture used in combination with Fresnel zone plates limits the most the experimental setup. Typical distances of the OSA from the sample position are in the range of few centimeters for hard Xrays.

\section{II.3 Laue microdiffraction}

The use of micron-sized white beams to perform local Laue diffraction was pioneered at the Advanced Photon Source (APS) in the late 90s and early 2000s [145, 146]. It was soon applied to the evaluation of defects in crystals [147] and extended from a 2D projection to a full 3D investigation [148]. Spurred by the need for stress mapping at the micron and submicron scale in microelectronics components Laue microdiffraction was implemented at Advanced Light Source (ALS) in Berkeley during the same period [149]. Very quickly innovative results on stress distributions in metal lines and thin films were obtained $[150,151]$ showing that very large stress inhomogeneities can be expected in these polycrystalline structures. The development of Laue microdiffraction came to Europe five years later with a first publication from the Swiss Light Source in 2006 [14]. During the same year, a station dedicated to Laue microdiffraction started working at the BM32 beamline at ESRF in Grenoble [152].

While coherent X-ray diffraction uses a monochromatic beam, Laue diffraction is based on polychromatic X-ray beams covering a large energy range. As described before, a convenient way for visualizing diffraction conditions is the construction of the Ewald sphere. With monochromatic X-rays the Ewald sphere passes only through a limited number of reciprocal lattice points (as shown in Fig. 24) making it necessary to rotate the sample to measure different Bragg reflections. However, when employing a broad spectrum of X-rays, i.e. a polychromatic beam, a large number of reciprocal lattice points are accessible simultaneously as indicated by the shaded area in Fig. 31. The two circles indicate the respective Ewald sphere for the X-rays with the largest energy (shortest wavelength) and the lowest energy (longest wavelength) within the spectrum of the polychromatic X-ray beam. All reciprocal lattice points which are in between these two circles (indicated by the shaded area) are measured simultaneously without any rotation of the crystal. This technique using a white (or polychromatic) X-ray beam is known as Laue diffraction which is historically the very first diffraction technique that has been employed in fact by Max von Laue. Thanks to the large number of diffraction 
spots recorded in one pattern the crystalline structure as well as the orientation of the crystal is readily available as will be shown below.



Fig. 31: Schematic illustration of the Ewald spheres for polychromatic X-ray beams covering a large number of reciprocal lattice points. The red and blue dashed circles represent the Ewald sphere for the lowest (longest) and the highest (shortest) $\mathrm{X}$-ray energy (wavelength) within the polychromatic beam.

Considering two parallel X-ray beams of different energy with the wave vectors $k_{\text {in }}$ and $k_{\text {in }}{ }^{\prime}$ being scattered on atomic planes of the same family, e.g. $\{111\}$ and $\{222\}$ planes, the wave vectors of the diffracted X-rays $\vec{k}_{\text {out }}$ and $\vec{k}_{\text {out }}$ ' are again parallel to each other (Fig. 32(a)). Thus, together with the two scattering vectors $\vec{q}$ and $\vec{q}^{\prime}$ the wave vectors of the incident and the diffracted X-rays form similar diffraction triangles. Since the diffraction pattern is recorded in the far-field, the two parallel diffracted beams are recorded at the same position on the detector. Since most detectors used for Laue diffraction do not have any energy resolution, the different energies of the impinging X-rays cannot be distinguished. Thus, the intensities from the diffracted beams are simply added up. This implies on the one hand that the measured intensity is the integrated intensity over a certain $q$ direction in reciprocal space (Fig. 32(b) [153]) and, on the other hand, the position of the Bragg peak in the diffraction pattern only depends on the relative direction of the atomic plane $\vec{a}_{q}$ with respect to the incident beam.

As a result of these conclusions, three important aspects can be deduced for Laue diffraction.

1. Each diffraction peak observed in the Laue diffraction pattern may be composed of several Bragg reflections, e.g. the diffraction peak indexed with (111) planes may be composed of several Bragg reflections from (111), (222), (333) planes, since their diffraction vectors are parallel to each other.

2. A homogeneous lattice expansion leads to equivalent changes of the primitive reciprocal lattice vectors. Thus, all reciprocal lattice points change along their $q$ directions as shown by 
the red line in the Fig. 32(c). Since the direction of all atomic planes $a_{\mathrm{q}}$ remains the same, no changes are observed in the diffraction pattern. Hence, for white-beam Laue diffraction, the unit cell volume cannot be uniquely determined preventing the measurement of the full strain tensor $\overline{\bar{\varepsilon}}$ (except in few special cases where a reliable mechanical model can be plugged into the data analysis [154]). Therefore, standard Laue diffraction only gives access to the deviatoric strain $\overline{\bar{\varepsilon}}_{\text {dev }}$ without the hydrostatic term $\overline{\bar{\varepsilon}}_{\text {hyd }}$

$$
\overline{\bar{\varepsilon}}_{\text {dev }}=\overline{\bar{\varepsilon}}-\overline{\bar{\varepsilon}}_{\text {hyd }}=\left[\begin{array}{lll}
\varepsilon_{11} & \varepsilon_{12} & \varepsilon_{13} \\
\varepsilon_{21} & \varepsilon_{22} & \varepsilon_{23} \\
\varepsilon_{31} & \varepsilon_{32} & \varepsilon_{33}
\end{array}\right]-\left[\begin{array}{ccc}
\Delta / 3 & 0 & 0 \\
0 & \Delta / 3 & 0 \\
0 & 0 & \Delta / 3
\end{array}\right]
$$

where $\Delta$ is the trace of $\varepsilon$, i.e. $\Delta=\varepsilon_{11}+\varepsilon_{22}+\varepsilon_{33}$. In order to measure the complete strain tensor, the energy for at least one Bragg peak has to be determined. This might be achieved either by using energy dispersive detectors such as a pnCCD $[155,156]$ or a fluorescence detector [157], or by the so-called rainbow method [158, 159, 160]. A pnCCD consists of energy dispersive pixels which allow for measuring energy spectra of the diffracted X-ray beam for each pixel giving an energy resolution of about $20 \mathrm{eV}$ at an $\mathrm{X}$-ray energy of $10 \mathrm{keV}$ (corresponding to a strain resolution of $0.2 \%$ ). The rainbow method bases on the introduction of a monochromator crystal in front of the sample which filters out a preselected energy. Due to this energy filter the intensity of certain Laue spots diminish allowing for correlating the diffraction spots with the energy of the scattered X-rays. This technique gives an energy resolution in the range of $1 \mathrm{eV}$ and, thus, a strain resolution of about $10^{-4}$ [161].

3. Contrary to homogenous lattice expansions, small rotations as shown in Fig. 32(d) result in displacements of the reciprocal lattice vectors perpendicular to their $q$ direction. Hence, the rotation of the crystal lattice which leads to directional changes of the atomic planes causes a movement of the diffraction spots on the detector. Therefore, the rotation of a crystal is directly accessible by Laue diffraction. 

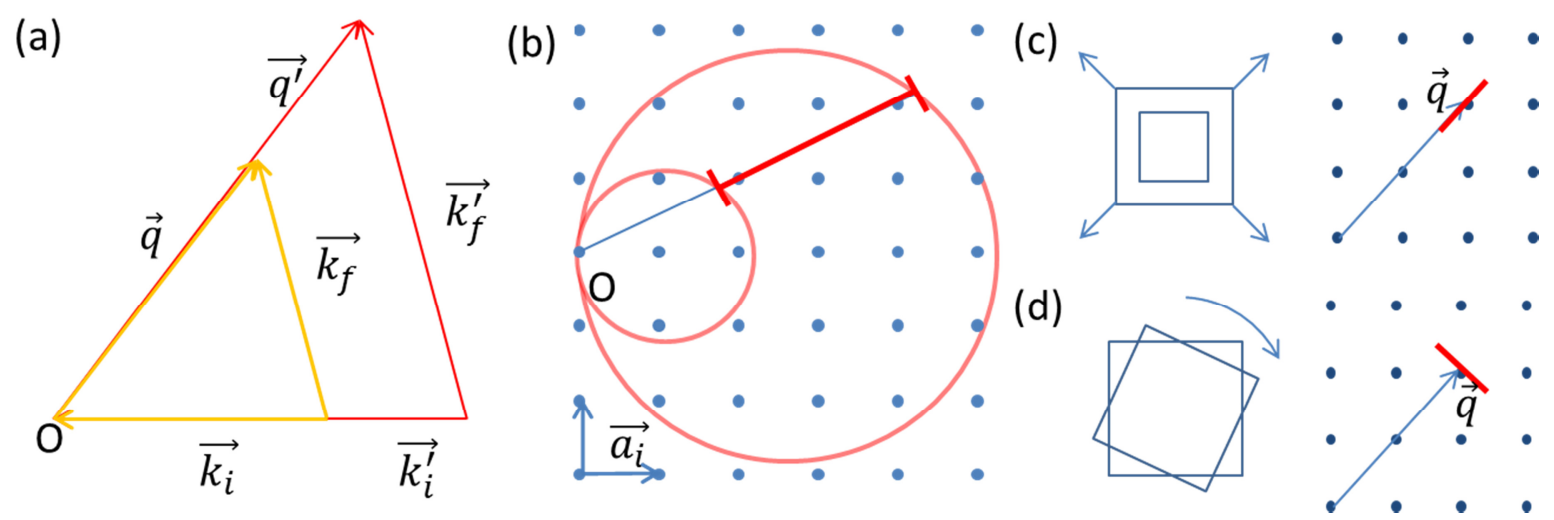

Fig. 32: a) Schematic illustration of two parallel diffraction vectors and their respective wave vectors. b) Two Ewald spheres for the minimum and maximum X-ray energy within a polychromatic X-ray beam. The red line illustrates the integrated intensity along a certain $q$ direction in the reciprocal space. Changes of the reciprocal lattice vector induced by c) a homogenous lattice expansion and d) a rotation of the crystal lattice [153]. While Laue microdiffraction cannot detect the former deformation, it is highly sensitive to any the crystal rotation.

Besides the crystal structure, the orientation, and the deviatoric strain, Laue diffraction also allows for detecting and determining crystal defects (as illustrated in Fig. 33) such as:

1. Point defects and coherent precipitates lead to a sharp but weaker Bragg reflection accompanied by some redistributed broad diffuse scattering intensity.

2. Statistically stored dislocations (SSDs) where the total Burgers vector equals zero cause a broadening of the diffraction peak in every direction and the diffraction peak exhibits a symmetric (circular) shape.

3. In contrast, geometrically necessary dislocations (GNDs) - unpaired dislocations - with a nonzero net Burgers vector induces a local lattice curvature. Thus, the orientation of the lattice planes and therefore also the diffraction vector slightly changes the orientation. This orientation gradient results in a streaking of diffraction peaks. By analyzing the streaking direction of the Laue spots the type of GNDs may thus be deduced. However, if dislocations with different Burgers vectors are present in a crystal the evaluation of the activated slip systems is ambiguous.

4. Dislocations form cells and boundaries to minimize the internal strain energy. Sub-grains which are separated by such boundaries can be observed with Laue diffraction and are evidenced by split intensities. 



perfect crystal
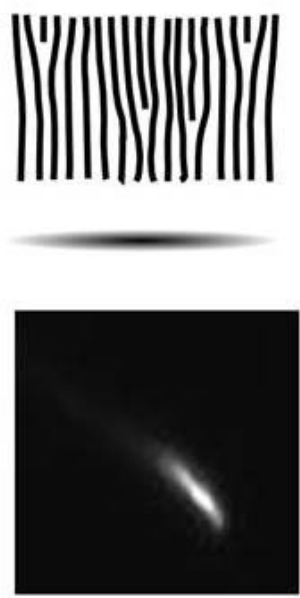

single slip


subgrain formation
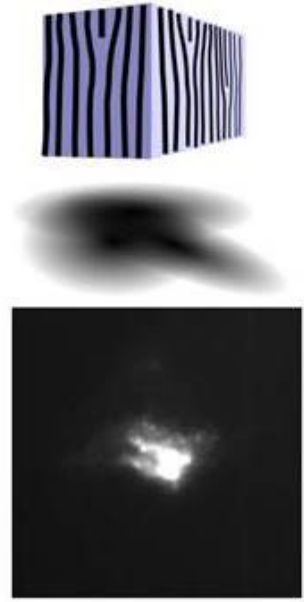

multiple slip

Fig. 33: Schematic illustration of the evolution of the shape of Laue peaks as a function of the crystalline quality [162].

For a given Laue diffraction pattern, it has firstly to be indexed by assigning Miller indices to each Laue spot before being able to determine the crystal orientation as well as the strain. Various programs such as XMAS [163], LaueTools [164], and LaueGo [165] have been developed in the recent past for these purposes. For indexing, first the primitive reciprocal lattice vectors are re-expressed by Cartesian coordinates using a transformation matrix B. In a second step, this reciprocal Cartesian coordinate system is rotated into a laboratory Cartesian coordinate system employing a matrix $U$ whose axis directions are related to the directions of the incident X-ray beam and the detector. Thus, by multiplying the Miller indexes with both matrixes $\mathrm{U}$ and $\mathrm{B}$, the directions of the reciprocal lattice vectors can be expressed in the lab frame.

In order to calculate the deviatoric strain tensor in the real space the primitive reciprocal lattice vectors are also expressed in Cartesian coordinates with a transformation matrix A. The transpose of this matrix A multiplied with matrix $B$ results in the identity matrix $I$. By means of a transformation matrix $T r$, this matrix $A$ of the distorted crystal can be further linked to the matrix $A_{0}$ from the perfect lattice structure: $A=\operatorname{Tr} A_{0}$. This transformation matrix contains both distortional and rotational components. Since the rotational component is antisymmetric, the distorted term can be obtained by

$$
\bar{\varepsilon}_{d e v}=\frac{T r^{t}+T r}{2}-I
$$

where $\overline{\bar{\varepsilon}}_{\text {dev }}$ is the deviatoric strain tensor of the crystal. 
In conclusion, the indexing of the Laue diffraction pattern consists of finding two matrixes $U$ and $B$ which are related to the orientation of the crystal and the lattice parameters, respectively, and which allow for determining the direction of the diffraction planes and the deviatoric strain tensor.

Inhomogeneous strain distributions and defects which lead to inhomogeneous displacement fields within a material result in changes in the Laue diffraction patterns. The effect of different defects on the Laue diffraction patterns are rather complicated and well summarized in a recent book by Barabash and Ice [166]. Here only a simplified view is given, where the influence of dislocations and inhomogeneous elastic strain on the Laue diffraction pattern is discussed on several examples.

An inhomogeneous strain distribution on the lattice rotation can be illustrated by the bending of a beam where the upper surface of the beam is compressively strained, while the lower surface is under tension. This inhomogeneous strain distribution leads to the rotation of the crystal lattice along the beam. These orientation gradients can be deduced either from the streaking of the Laue spots or from the movement of the diffraction peaks between neighboring positions of the illuminated sample volume which is given by the footprint of the incident $\mathrm{X}$-ray beam which is typically of the order of $500 \mathrm{~nm}$ [167]. Besides this inhomogeneous elastic strain distribution, the storage of dislocations may also result in the rotation of the crystal lattice due to the inhomogeneous displacements generated by the dislocations in the crystal lattice. Considering the displacement around a single edge dislocation in an infinite isotropic elastic crystal [168] where the $x$ axis is along the Burgers vector and the $y$ axis is perpendicular to the slip plane the rotation is described by the following equation:

$$
\omega_{x y}=\frac{b x}{\pi\left(x^{2}+y^{2}\right)}
$$

It is worth noting that dislocations with different signs ("+" and "-") cause rotations in opposite directions. Thus, when dislocations with different signs are stored within the probed volume, the rotations from these dislocations cancel each other, which reduce or even eliminate the long-range rotation of the material. Therefore, when an equal density of dislocations of each sign is stored in a material, only a slight weakening and broadening of the Laue spots is observed in the diffraction pattern, which is difficult to detect by white beam Laue diffraction. Usually this kind of dislocation storage is found for uniaxial mechanical tests of bulk materials, where dislocations are statistically stored at different obstacles. This kind of stored dislocations, called statistically stored dislocations (SSD), is not detectable by white beam Laue diffraction.

In contrast to the SSDs, an excess number of dislocations of same sign are found in systems loaded in such a way that strain gradients develop. These dislocations are produced in order to 
reduce the strain energy in the crystal. Since rotations from dislocations of the same sign do not cancel each other but they accumulate resulting in macroscopic rotations which are detectable by Laue diffraction. Considering a Burgers circuit between two parallel slip planes, the closure failure is given by $n b$, where $n$ is the excess number of dislocation with one sign and $b$ is the Burgers vector of an edge dislocation. This closure failure corresponds to the elongation of one side of the slip plane and, thus results in a deformation of the crystal as a total as in the case of an elastic bending. Hence, dislocations of one sign are stored to accommodate the deformation from various parts of the crystal. Since the density of the stored dislocations is related to the geometry of the structure, this kind of dislocations is called geometrically necessary dislocations. Note that the concept of GNDs depends on the probed volume. For example, when just one dislocation is probed, every dislocation is a GND, like in the case of the recently reported paper by Hofmann et al. [169].

In order to detect the rotation caused by GNDs two methods exist. When the volume in which the GNDs are stored is illuminated, streaked diffraction peaks are observed while when the volumes on each side of the stored GNDs are probed separately, the rotation induced by the GNDs leads to the movement of the diffraction spots on the detector. It is worth noting that instead of yielding rotations in arbitrary crystal directions, rotations induced by GNDs are around specific crystalline directions, which correspond to different slip systems. For example, for the $<110>\{111\}$ type of dislocation in a FCC crystal, the rotation axis from different slip systems is always around the <112> direction. Thus, by measuring the rotation by means of Laue diffraction and comparing them with the different $<112>$ rotation axis, the activated slip systems can be identified. Finally, it is worth noting that when dislocations glide through the crystal, the crystal lattice restores to the perfect position and, thus no detectable change is observed by Laue diffraction.

In recent years, the Laue microdiffraction technique has been further developed. On the one hand, it has been combined with digital image correlation (DIC) that allows for tracking the movement of the Laue spots on the detector with a subpixel resolution $[170,171]$. This method has been shown to be suitable to measure Laue spot motion between different deformation states and to deduce the associated increment of lattice rotation and of local elastic strain which typically induce displacements of the Laue spots smaller than a pixel on the detector. With Laue-DIC strain resolutions of $10^{-5}$ and thus stress resolutions of the order of few MPa can be obtained [170, 171, 172]. On the other hand, a 3D Laue microdiffraction approach has been developed which allows the measurement of the dislocation densities as well as stress and strain with sub-micrometer resolution in three dimensions $[167,173,174]$. This technique bases on differential-aperture X-ray microscopy (DAXM) which exploits knife-edge step profiling to provide a micrometer-resolution X-ray slit with high angular acceptance in combination with a $2 \mathrm{D}$ detector. This approach is analogous to a 
translating pinhole camera providing access to full diffraction information from sub-micrometer voxels in bulk materials with strain resolutions of the order of $10^{-4}$.

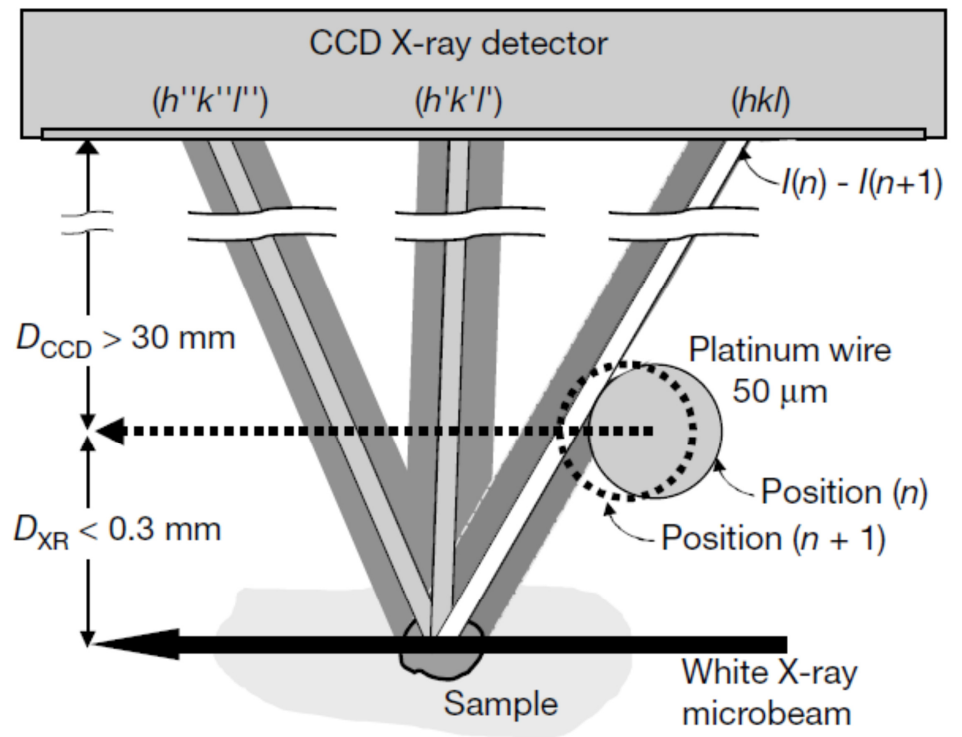

Fig. 34: Differential-aperture X-ray (structural) microscopy depth-profiling method. Schematic view of a white microbeam penetrating a sample and scattering into a CCD area detector. Bragg scattering for the (hkl), (h'k'l') and (h"'k'l"'") Laue reflections is depicted for a single grain and for a small segment (or voxel) in the interior of the grain. A Pt wire profiler is shown at position (n) and the dashed circle indicates the $(n+1)$ position of the wire after a sub-micrometer step. Taken from ref. [173].

Fig. 34 illustrates the method for obtaining depth-resolved Laue patterns from bulk samples. After collecting a CCD image with the wire at position $n$, the wire is stepped to position $n+1$ where a second CCD image is collected. The source of the differential intensity $I(n)-I(n+1)$ in each pixel of the detector can be uniquely assigned to length segments along the microbeam using the position of the profiling wire and the position of individual CCD pixels. This is illustrated by the intensity of the $\mathrm{hkl}$ reflection emanating from a voxel in the center of the grain shown in Fig. 34. Continuing the process of stepping the wire across the diffraction pattern, the corresponding differential intensities for the $h^{\prime} k^{\prime} l^{\prime}$ and $h^{\prime \prime} k^{\prime \prime} l^{\prime \prime}$ reflections are generated in a similar manner. Comparison of the pixel-bypixel differential intensities provides a spatial mapping with sub-micrometric resolution of the entire Laue diffraction pattern onto the source of the scattering along the incident microbeam. The pixel positions, the geometrical parallax associated with the $\left(\sim 20 \times 20 \mu \mathrm{m}^{2}\right)$ pixel size, and the step size, step direction, and the circular shape of the profiling wire are taken into account. The wire may be stepped at an angle to the microbeam if desired. It is necessary for the overall precision and accuracy of the method that the distance of the wire to the sample $D_{\mathrm{XR}}$ is much smaller than the distance to the detector $D_{\text {CCD }}$. This condition ensures that the parallax correction due to CCD pixel size is small and that the spatial resolution of the reconstructed diffraction patterns is determined primarily by 
the step size of the profiling wire differential aperture. Therefore, depth resolutions significantly smaller than a micrometer is readily achievable.

Fig. 35 depicts the geometry for depth-dependent measurements in a 25 - $\mu \mathrm{m}$-thick <001> oriented Si plate with $\langle 110>$ edges that was bent to a radius $R=3.3 \mathrm{~mm}$ at the apex of the arch. While standard Laue microdiffraction (depth-integrated Laue) patterns exhibit streaked Laue spots, depth resolved Laue microdiffraction allows for measuring split diffraction spots which originate from the upper and the lower surface of the bent Si plate. These measurements eventually facilitate the determination of the deviatoric strain tensor for the compressed and the stretched parts of the sample.

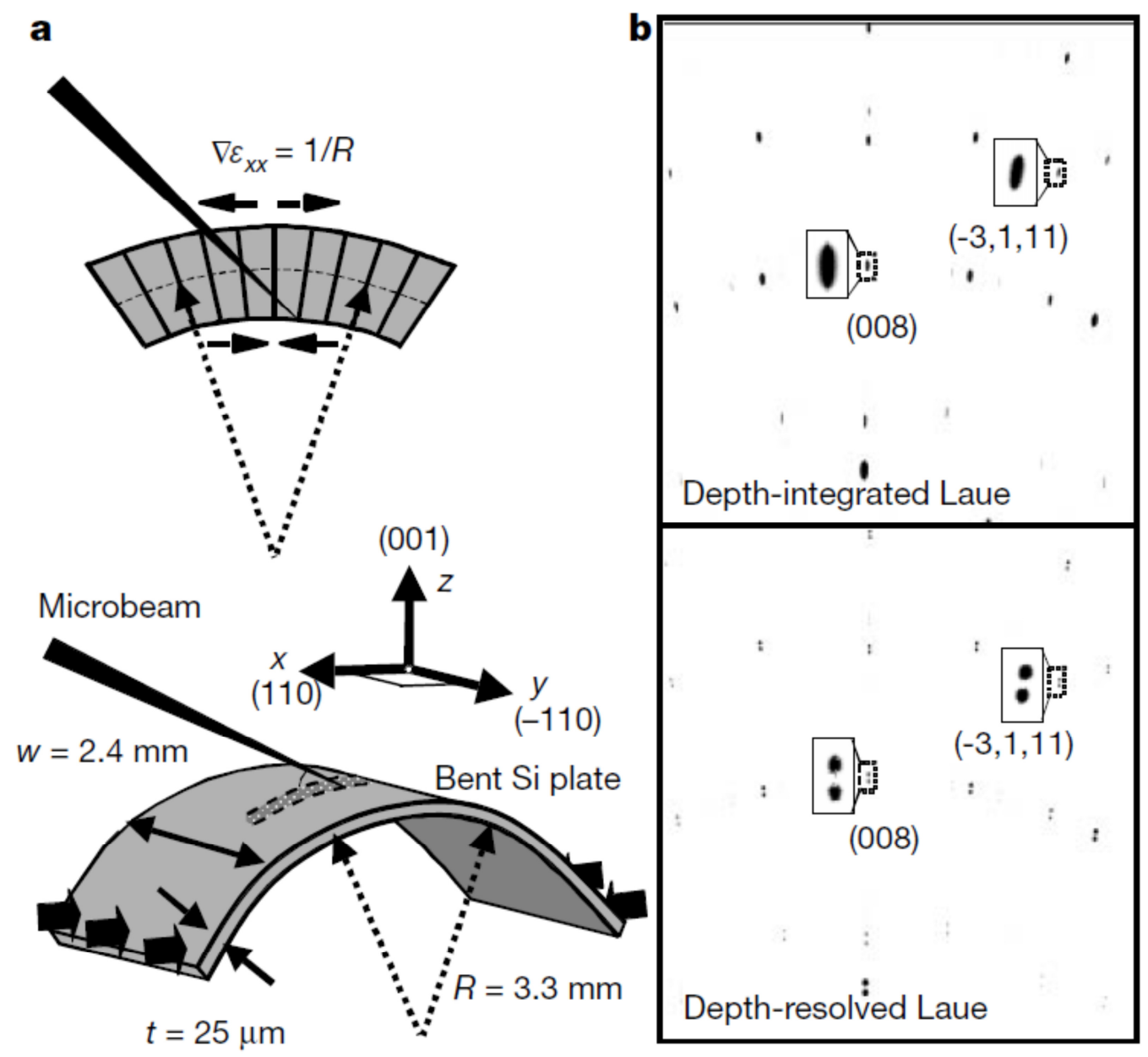

Fig. 35: DAXM orientation and strain measurements in cylindrically bent Si plate. a) Illustration of the strain gradient in a thin, bent plate and the $\mathrm{X}$-ray microbeam geometry used for strain tensor measurements. b) Polychromatic microbeam diffraction pattern from cylindrically bent Si (top panel), and depth-resolved diffraction patterns extracted by DAXM for $1-\mu \mathrm{m}$ layers near the top and the bottom surfaces of the plate (bottom panel). The enlarged views for the 008 and $-3,1,11$ reflections indicate the effects of bend-induced strain and the $\left(0.01238^{\circ}\right.$ per $\left.\mu \mathrm{m}\right)$ bend-induced rotations along the beam direction. Taken from ref [173]. 


\section{II.4 Coherent Bragg diffraction}

\section{II.4.i Coherence}

As described in section II.1 and formula (15) the amplitude scattered by a crystal is a Fourier transform of electron density times a complex phase factor, which is related to the projection of the displacement field on the Bragg vector. This has been derived under the implicit assumption that the crystal is illuminated by a plane wave i.e. a fully coherent beam. Highly brilliant and highly coherent $X$-ray beams are nowadays generated at $3^{\text {rd }}$ generation synchrotron sources. In a simple approach the coherence of a wave can be described via two different coherence lengths - longitudinal and transversal. They depend on the monochromaticity of the X-ray beam and on the source size seen from the scattering object. Considering a point source emitting monochromatic radiation, the coherence lengths are infinite. The longitudinal coherence length is defined as

$$
L_{c}=\frac{\lambda^{2}}{2 \Delta \lambda}=-\frac{h c}{2 \Delta E}
$$

Considering a typical Si 111 monochromator which exhibits an energy resolution of $\Delta E / E \sim 10^{-4}$ results in a longitudinal coherence length of the order of $0.6 \mu \mathrm{m}$ for $10 \mathrm{keV} \mathrm{X-rays.}$

On the other hand, the $\mathrm{X}$-ray sources are not point-like but they have a finite size. X-rays emitted from two different regions of the source do not have any phase relation. When considering Young's double-slit experiment using two point sources, which are separated by a distance $S$, the two interference patterns created by the waves emitted from the two sources overlap. In order to maintain an interference pattern, the phase shift between the two patterns must be less than $\pi$ resulting in a lateral coherence length defined by the following equation

$$
L_{c}=\frac{\lambda D}{2 S}
$$

where $D$ is the distance of the source from the double-slit. Therefore, the lateral coherence lengths increase with decreasing source size and increasing sample-source distance which reduces the effective source size. Typical values of the lateral coherence lengths for $3^{\text {rd }}$ generation synchrotron beamlines are few tens of micrometers in the horizontal and around $100 \mu \mathrm{m}$ in the vertical direction. Several upgrade programs are underway at $3^{\text {rd }}$ generation synchrotron sources. They rely on the development of hybrid multi-bend achromats which will allow for a significant decrease in horizontal emittance. The resulting gains in brilliance and coherent flux are expected to revolutionize coherent diffraction imaging. 


\section{II.4.ii Phase retrieval}

Note that in all X-ray diffraction experiments, diffraction intensities are measured. While the amplitude of any electromagnetic wave is described by its modulus $A_{0}$ and the phase, the intensity is the complex square of the amplitude. This leads to the so-called phase problem, namely that the phase is lost in the measurement. Since the phase of the scattered amplitude is not measured one uses generally model-dependent approaches. The square modulus of the Fourier transform from a model structure is compared with the experimental intensity. Direct inversion, based on phase retrieval algorithms can, however, be performed. It is based on the oversampling conception [175], which states that the diffracted intensity pattern should be sampled at a frequency at least twice the highest spatial frequency in the object.

Great progress has been made with respect to phase retrieval algorithms which - as their name already suggests - allow for retrieving the phase of the diffracted beam, and thus, measuring the strain field in the structure under study. All algorithms base on an iterative approach of Fourier transformations (FT) and inverse Fourier transformations $\left(\mathrm{FT}^{-1}\right)$ and to apply certain constraints in the real as well as in the reciprocal space. This approach is schematically illustrated in Fig. 36.
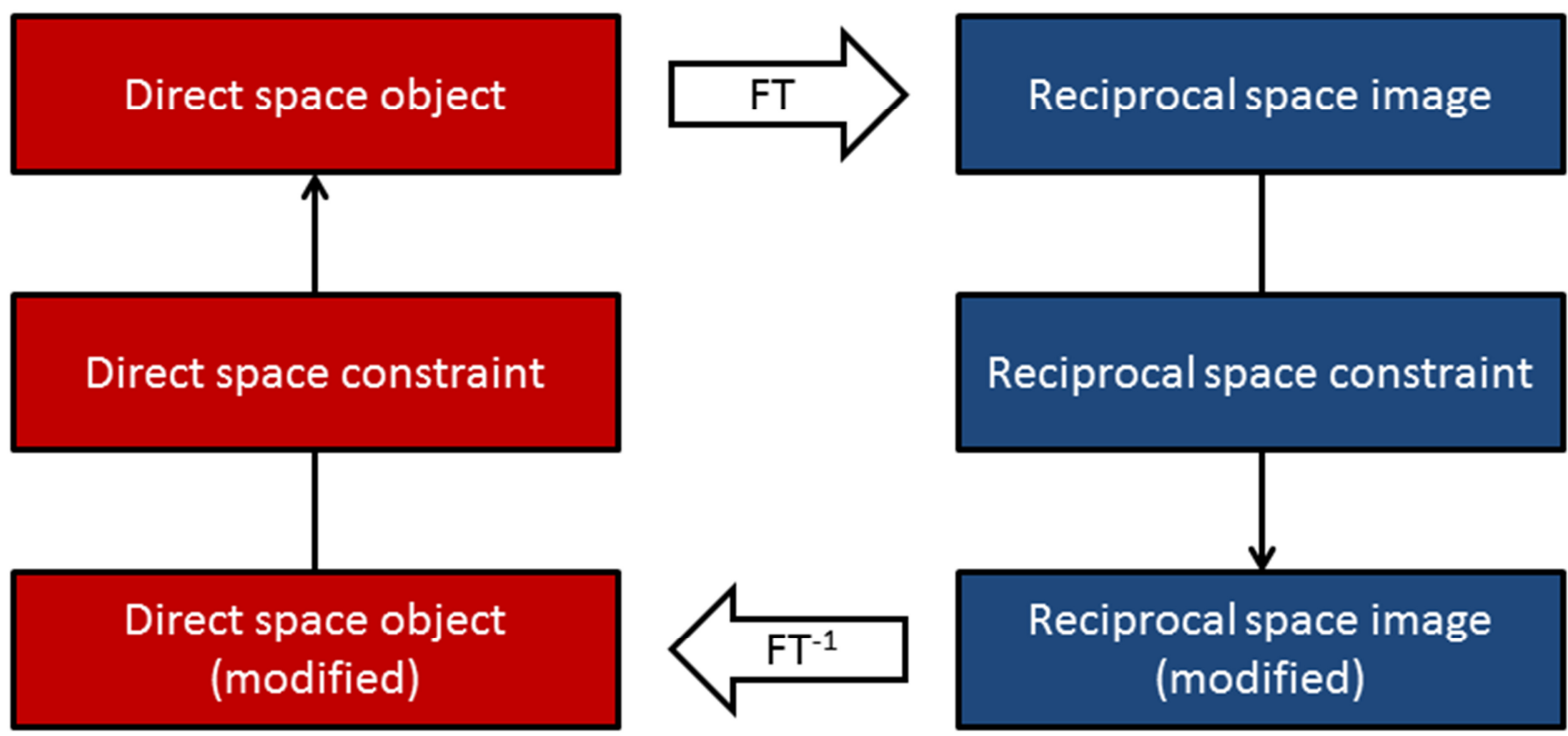

Fig. 36: Schematic illustration of the error reduction phase retrieval algorithm which starts with an arbitrary guess and contains several iterations between the real and the reciprocal space by means of Fourier and inverse Fourier transformations applying constraints in each space.

Firstly, the square-root of the measured intensity is used as the measured amplitude which is multiplied with a random phase. After an inverse Fourier transformation, certain constraints are applied on the obtained real space object such as defining the sample support. This new direct space image is Fourier transformed and the computed amplitude is replaced by the measured amplitude $(\sqrt{I})$. These algorithms were further improved in recent years by developing new algorithms or 
improving existing ones such as the error-reduction (ER), the hybrid input-output (HIO), and the shrink-wrap (SW) algorithm.

The ER algorithm was developed by Gerchberg and Saxton [176] and it was further improved by J.R. Fienup who proposed the use of real-space constraints introducing a finite support instead of intensity measurements which are allowed only in the reciprocal space $[177,178,179]$. At each projection this algorithm looks for the closest point satisfying the set of constraints and the distance between two sets is minimized. It starts with an initial guess, corresponding to the measured amplitude. A first estimation of the real space object is obtained applying an inverse Fourier transformation to the initial guess to which a random phase is added. As constraints in real space all data outside the support are set to zero and for undeformed objects the reconstructed real-space density has to be positive. The constrained object is Fourier transformed and the reconstructed amplitude is replaced by the measured amplitude. These steps are repeated until the algorithm converges which is assured by introducing a metric error, usually obtained looking at the autocorrelation of the diffraction pattern [180]. Note that the ER algorithm easily gets trapped in local minima and it is not sufficient to reconstruct the object in real space due to the low convergence. In order to avoid stagnation of the ER algorithm, J.R. Fienup developed the HIO algorithm in which the real space constraint is obtained by the combination of the object from the previous (input) and the current (output) iterations [177, 178, 179]. It aims therefore on minimizing the error using this combination. A large amount of variations in the next input is produced and at the same time stagnation is avoided. A common algorithm used nowadays for the reconstruction of the phase in coherent diffraction experiments is the shrink-wrap algorithm which has been introduced by Marchesini et al $[181,182]$. With respect to the HIO and the ER algorithm, the object support is not fixed but it is updated during the reconstruction and, thus it is determined together with the object itself. The algorithm starts with a support that fits the first estimation of the object in real space. While this first estimate of the support is obviously not accurate, the intensity in real space is selected applying a threshold, thus updating the support until the reconstructed object fits within the autocorrelation function. Consequently, the support "shrinks" progressively around the object "wrapping" all its volume.

\section{II.4.iii Bragg coherent diffraction imaging (BCDI)}

With the advent of $3^{\text {rd }}$ generation synchrotron sources and the increase of both the brilliance and the coherence of the provided synchrotron X-ray beams as well as the progress on focusing optics, a new lens-less imaging technique the Bragg coherent X-ray diffraction imaging (BCDI) method started to being developed. In 2003 , the first successful reconstruction of a Au nanocrystal from 
experimentally obtained coherent X-ray diffraction data was reported (see Fig. 37(a)) [128, 183]. The first successful phase retrieval was demonstrated on Pb nanocrystals in 2006. Phase maps cutting through the nanocrystal are presented in Fig. 37(b)) [43].

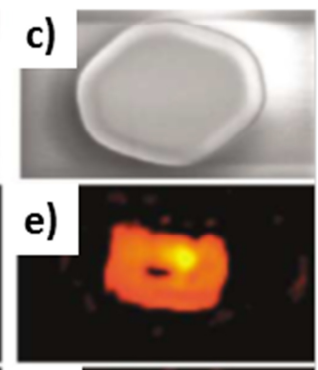

g)



h)
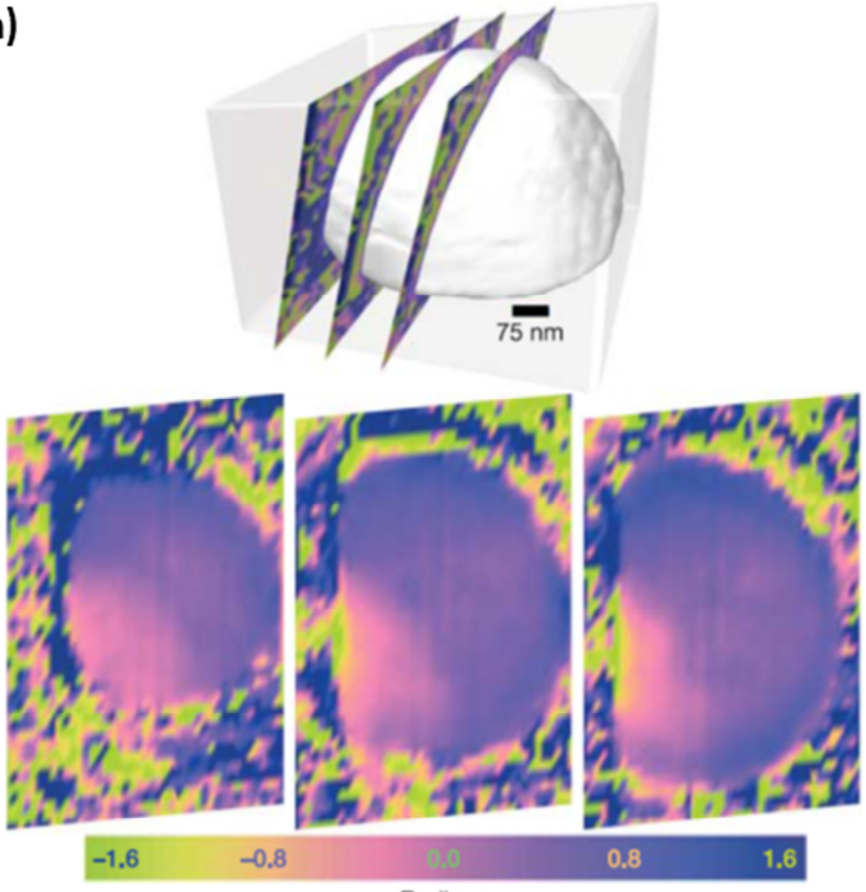

Radians

Fig. 37: a) Bragg coherent X-ray diffraction pattern of a Au nanocrystal. b), c) SEM images of Au particles. d) Size of the "support" constraint used in the inversion routines. e) - g) Real-space images obtained by inversion of the diffraction patterns [128]. h) Phase maps cutting through a Pb crystal at three parallel planes. Schematic diagram of the sections which are $138 \mathrm{~nm}$ apart. The translucent box is the support region used in the phasing calculations, which was rectangular before the coordinate transformation. The phase bulge is interpreted as a projection of strain fields in the crystal lattice arising from contact forces at the interface with the substrate [43].

The experimental techniques as well as the phase retrieval algorithms were further improved over time and, in 2009, Newton et al. demonstrated the determination of the complete strain tensor from coherent Bragg diffraction imaging of six independent Bragg peaks on a single ZnO nanorod [184]. A scanning electron microscopy image of the $\mathrm{ZnO}$ nanorod under study, the measurement configuration, and the strain maps for the six independent components of the strain tensor are presented in Fig. 38(a), (b), and (c). The spatial resolution of this reconstruction amounted to $40 \mathrm{~nm}$. 
a)

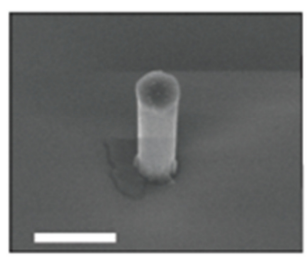

b)

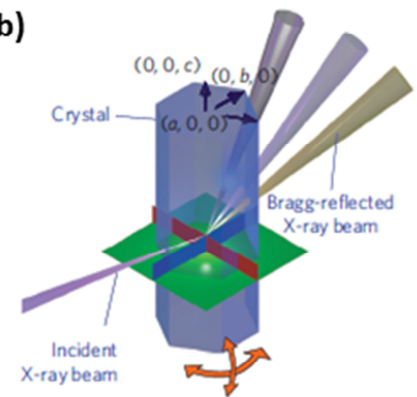

c)

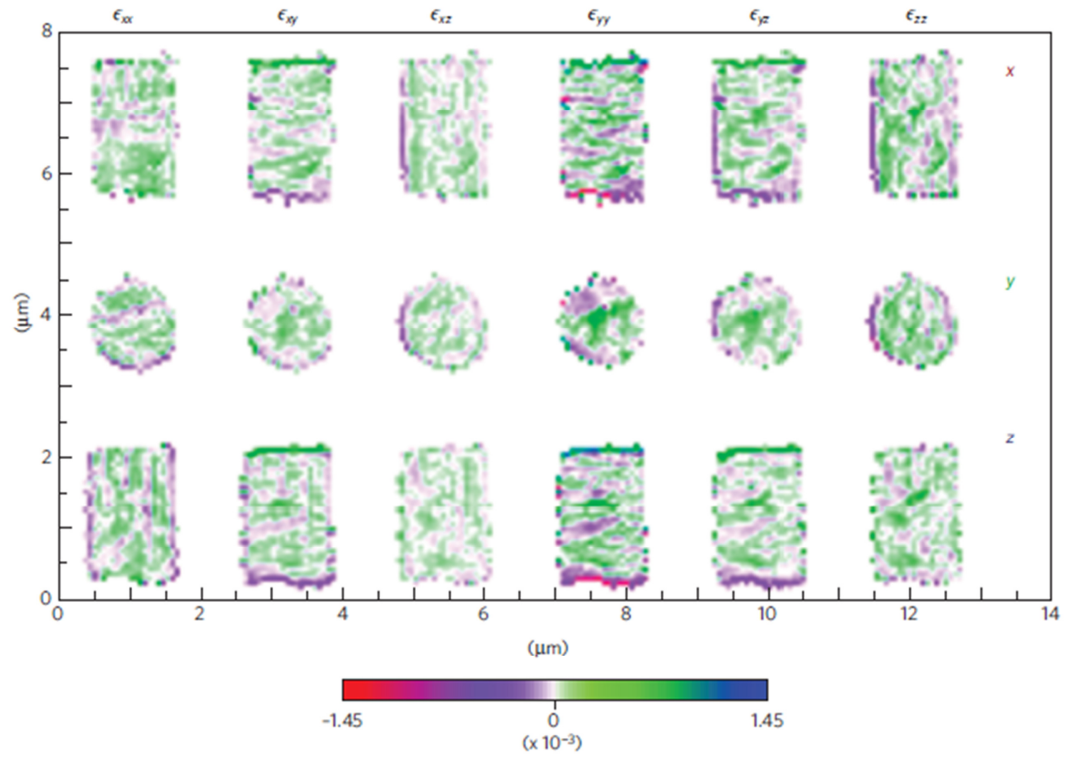

Fig. 38: a) Scanning electron micrograph of a $\mathrm{ZnO}$ nanopillar (scale bar: $2 \mu \mathrm{m}$ ) and b) schematic of the experimental configuration showing multiple Bragg reflections from a single $\mathrm{ZnO}$ rod. c) Twodimensional slices of the six independent components of the strain tensor. Components are taken along the three Cartesian axes according (b). Regions of compressive (negative) strain are observed near the (100) surfaces of the crystal and at the interface with the Si substrate. A strained layer approximately $200 \mathrm{~nm}$ in width near the surface and along the length of the rod $(y$ axis) is visible in all tensor components except the $\varepsilon_{y y}$ component. This implies that the strain is uniform along the length of the rod. [184].

While the measurements described above concentrated on weakly deformed objects without any defects, Jacques et al. demonstrated the imaging of a dislocation in Si by coherent X-ray diffraction [185]. Recent works focus on nanostructures, which contain one single defect as well as on highly strained objects, and nanostructures containing dislocation networks [186, 187, 188, 189, 190]. Favre-Nicolin et al. reported on Bragg coherent diffraction imaging studies on single GaAs nanowires revealing a bar-code pattern, which originates from stacking faults within the nanostructure [186]. Recently, Labat et al. demonstrated the reconstruction of GaN nanowires containing inversion domain boundaries measuring five independent Bragg peaks of the same nanorod [187]. The authors reported a displacement of $8 \mathrm{pm}$ along the c-direction with an accuracy of 1 pm demonstrating the very high accuracy of measuring displacement fields by BCDI (see Fig. 39). 

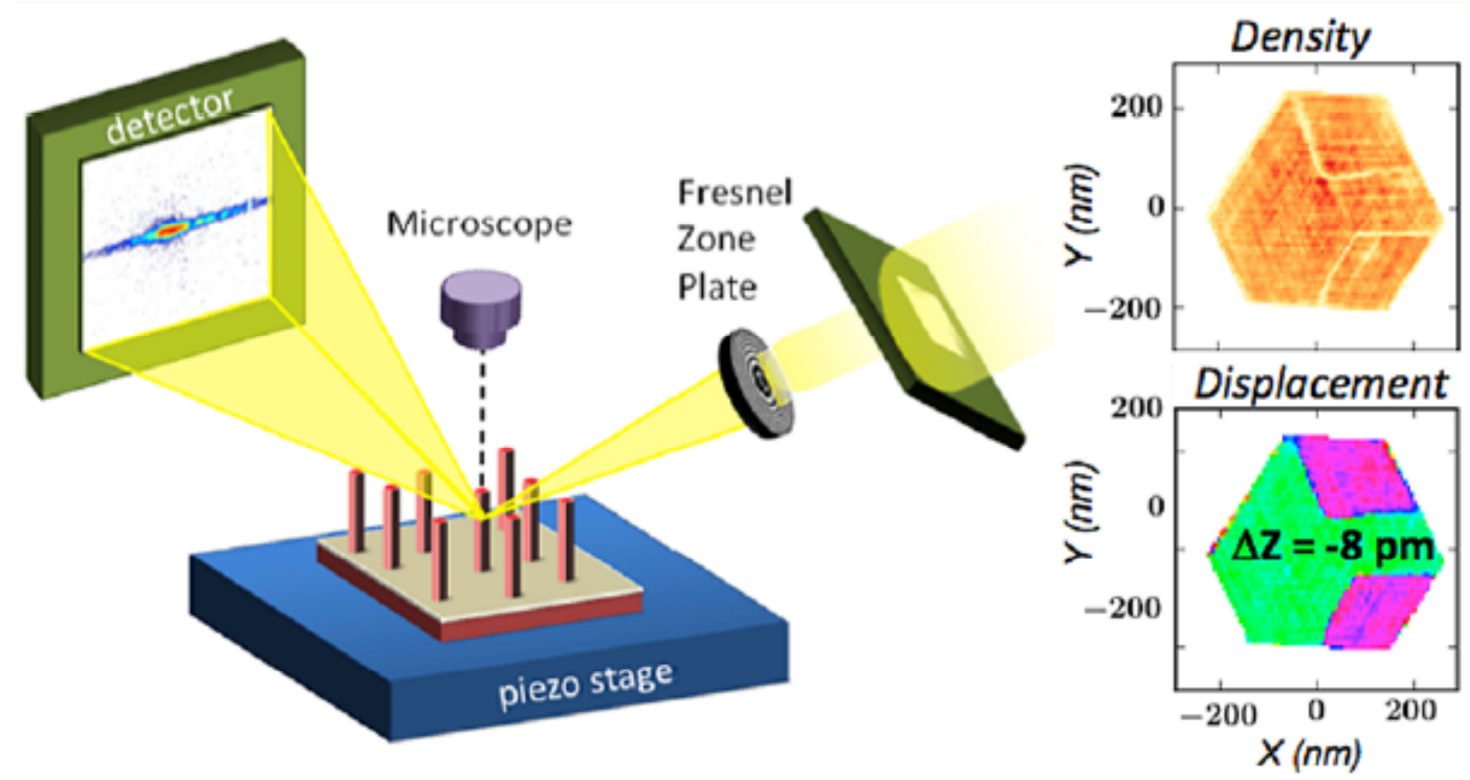

Fig. 39: Schematic illustration of the experimental configuration for BCDI on a single GaN nanopillar containing inversion domain boundaries. The incident $X$-ray beam is focused by a Fresnel zone plate and the diffracted X-rays are recorded by a two-dimensional pixel detector. The sample is mounted on a piezoelectric stage and an optical microscope facilitates the observation and alignment of it. Density and displacement field of the reconstructed object. The green and violets part a Ga-polar and N-polar zones within the nanopillar which are shifted along $z$ by $(c / 2-8 \mathrm{pm})$ with respect to each other [187].

Hofmann et al. further demonstrated the reconstruction of the shape and the phase of Au nanocrystals bombarded with $\mathrm{Ga}^{+}$ions in a focused ion beam microscope examining the defects caused by FIB milling in initially pristine objects [190]. The authors found that every use of FIB causes large lattice distortions even for very low ion doses which are typically used for FIB imaging and which were previously thought to be negligible. The reconstructed lattice displacement and lattice strain for a gold nano-crystal that was exposed to a $\mathrm{Ga}^{+}$dose of $4.2 \times 10^{4} \mathrm{ions} / \mu \mathrm{m}^{2}(30 \mathrm{keV}, 50 \mathrm{pA}$ ), which is sufficient to image the sample is shown in Fig. 40(a) - (c). The 3D field of lattice displacement reveals large displacement near the top implanted surface of the crystal. At larger ion fluences used during FIB milling extended dislocations networks, that cause stresses far beyond the bulk tensile strength of gold, were retrieved as demonstrated by the reconstructed lattice displacement (Fig. 40(d)) and lattice strain (Fig. 40 (e)) for a gold crystal exposed to a fluence of $1.3 \times 10^{7}$ ions $/ \mu \mathrm{m}^{2}$. The ordering of larger defects is visualized in Fig. 40(f) by showing the von Mises stress $>500 \mathrm{MPa}$ revealing an arrangement of defects in lines. These observations provide new fundamental insight into the nature of the damage created by FIB milling and defects that lead to inhomogeneous morphology. 


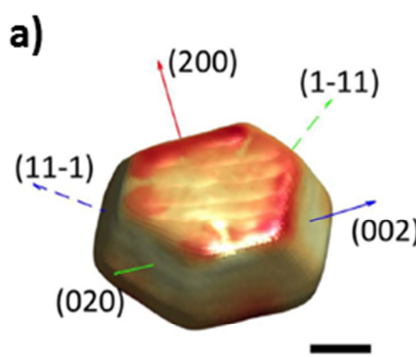

b) strain tensor in xy plane (red)

C) strain tensor in yz plane (green) $\times 10^{-3}$
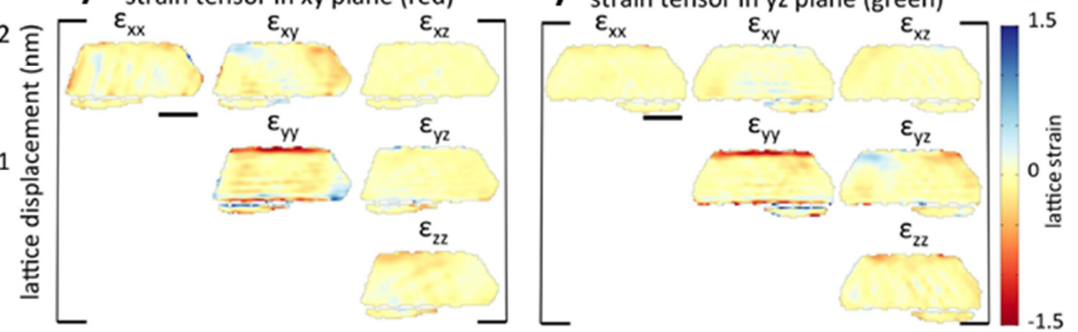

d)



$(11-1)^{\prime}$ e)

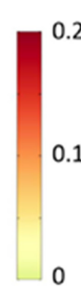

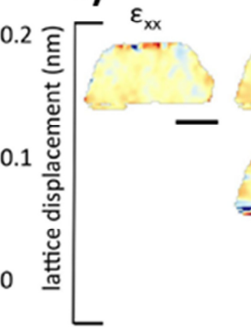


f)

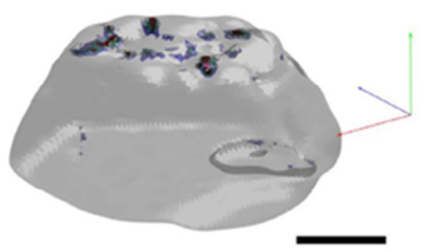

Fig. 40: a) 3D rendering of the crystal after FIB imaging with a $\mathrm{Ga}^{+}$ion fluence of $4.2 \times 10^{4} \mathrm{ions} / \mu \mathrm{m}$ colored by lattice displacement magnitude. Superimposed are the $q$ vectors of the five measured crystal reflections. Maps of the six independent lattice strain tensor components on b) the xy section and c) the yz section. d) 3D rendering of a crystal after FIB milling with a $\mathrm{Ga}^{+}$ion fluence of $1.3 \times 10^{7}$ ions $/ \mu \mathrm{m}^{2} \mathrm{coloured}$ by lattice displacement magnitude. $q$ vectors of the six measured reflections are superimposed. e) Maps of the six independent lattice strain tensor components plotted on the $x y$ section.f) Semi-transparent rendering of the outer crystal shape superimposed with iso-surface of the von Mises stress (300 MPa (blue), 400 MPa (green), 500 MPa (red)). Scale bars are 300 nm [190].

Besides "static" measurements, recent developments aim on imaging defects as well as the strain field in nanostructures operando. For example, Clark et al. visualized in three dimensions the entire network of dislocations within an individual calcite crystal and its propagation during repeated growth and dissolution cycles demonstrating the potential of Bragg coherent diffraction imaging for studying the mechanisms underlying the response of crystalline materials to external stimuli [188]. Similarly, Ulvestad et al. reported on the evolution of strain in a single $\mathrm{LiNi}_{0.5} \mathrm{Mn}_{1.5} \mathrm{O}_{4}$ particle which is used in lithium ion batteries during charging and discharging [191]. Bragg coherent X-ray diffraction imaging has a bright future thanks to the construction of new $3^{\text {rd }}$ generation synchrotron sources such as MAX IV and the upgrade of existing ones to extremely brilliant sources where ordinary bending magnets are replaced by multi-bend achromats, thus increasing the coherent flux by one to two orders of magnitude. The gain in coherent flux will significantly reduce the data acquisition time. Consequently, the synchrotron upgrades as well as X-ray free electron lasers will allow for further developing the BCDI technique and eventually enable time resolved measurements. Clark et al. already demonstrated first pump-probe experiments and imaged lattice dynamics in an individual nanocrystal [192]. 
These experiments as well as many others, which can be found in literature [44, 193, 194, 195] demonstrate the major progress that has been made within the last 15 years in the field of Bragg coherent diffraction imaging. This technique is very well suited to study the strain fields induced by mechanical loading as well as the onset of plasticity in circumstances where only a limited number of defects and dislocations are present in the crystal under study. The BCDI technique was extended to Bragg holography by Chamard et al. [196] as well as ptychography where the coherent beam is raster scanned across a sample with an overlap of at least $50 \%$ of the beam at adjacent positions. The latter approach facilitates the study of arbitrary large samples [135, 197, 198]. In addition, while in ordinary $B C D I$ experiments the wave front of the incident beam is a priori unknown, the wave front is directly obtained by the ptychographic approach thanks to the overlap of the beam.

The penetration depth of hard X-rays with an energy of $10 \mathrm{keV}$ reaching $100 \mu \mathrm{m}$ in Si allows for studying specimen that are too thick for transmission electron microscopy while offering at the same time strain resolutions of $10^{-4}$ and high sensitivity to defects and dislocations inside the structure. In addition, the enormous progress made on focusing optics at $3^{\text {rd }}$ generation synchrotron sources facilitates nowadays to focus hard X-ray beams down to the few hundred nanometer length scale. While this focal size defines the spatial resolution for "ordinary" X-ray diffraction methods or for Laue microdiffraction, Bragg coherent X-ray diffraction renders resolutions far better than the beam size reaching values of $<10 \mathrm{~nm}$. In addition, the latter offers unprecedented resolution of $1 \mathrm{pm}$ for the displacement field. The effective pixel size of the used X-ray detector, i.e. pixel size and distance of detector from sample position defines the oversampling that is necessary for phase retrieving the data in real-space (typically 2 pixels per fringe are needed [175]). The real-space resolution of the reconstructed data is related to the maximum spatial frequency recorded in the useful diffraction data from the central Bragg peak. It is limited, on the one hand, by the size and distance of the camera and by the incident coherent flux. But one must also consider the reliability of the recovered phases at the different spatial frequencies. Because the reconstruction is generally based on the average of many iterates, one measure can be obtained by comparing the magnitude of the Fourier transform of the averaged complex reconstruction with the magnitude of the recorded diffraction intensities as a function of spatial frequency. This provides a more complete indication of resolution than a single parameter [199]. 


\section{PROGReSS OF IN SITU MICRO- AND NANO-MECHANICAL TESTING IN COMBINATION WITH SYNCHROTRON X-RAY DIFFRACTION TECHNIQUES}

As demonstrated in the previous section, $\mathrm{X}$-ray diffraction methods are highly sensitive to elastic strain and defects. The structural properties of individual micro- and nanostructures are accessible thanks to the tremendous progress achieved on the focusing optics at $3^{\text {rd }}$ generation synchrotron sources. In addition, the comparatively large space available at synchrotron beamlines as well as the penetrating power of X-rays, in comparison to transmission electron microscopes, paved the way to the development of various in situ micro- and nano-mechanical testing devices in the recent past.

In 2007, the group of H. Van Swygenhoven at the Paul-Scherrer Institute (PSI) in Switzerland developed an indenter for in situ micro-compression tests on metal pillars in combination with Laue microdiffraction [200, 201]. In 2011, Kirchlechner et al. designed a similar instrument for in situ compression and bending tests on micrometric metal pillars in combination with Laue microdiffraction at the BM32 beamline at the European Synchrotron in Grenoble [202]. While the former device was designed for Laue microdiffraction studies in forward direction by applying the load vertically on the structure under investigation, the second tool was constructed so that the load is applied horizontally, thus facilitating studies in the vertical diffraction plane without the need of penetrating the sample. Besides Laue microdiffraction, the latter device was also coupled with coherent X-ray diffraction during compression tests of semiconductor micropillars [203].

An in situ indenter was recently developed at the P03 beamline at the PETRA III synchrotron source in Hamburg (Germany). As illustrated in Fig. 41, this instrument is coupled with X-ray diffraction in transmission geometry using hard X-ray beams that are focused down to a size of the order of $200 \mathrm{~nm}$ [204, 205]. The local mechanical deformation, the microstructural changes, and stress distributions can be studied during step-wise indentation of lamellas of several micrometer thick films as well as bulk metallic glasses. 


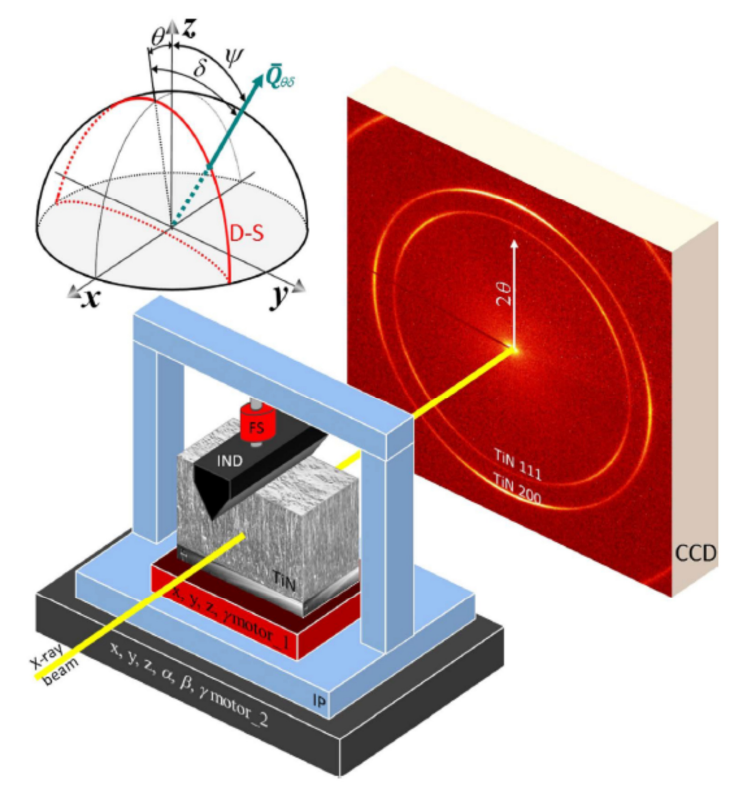

Fig. 41: Schematic of the in-situ X-ray nanodiffraction setup where a $9 \mu \mathrm{m}$ thick TiN film on a steel substrate is loaded against a diamond wedge indenter accompanied by a simultaneous measurement of the applied load by a force sensor. The sample is scanned through the X-ray beam and the $2 \mathrm{D}$ charge-coupled device (CCD) detector collects TiN Debye-Scherrer rings for each $y$ and $z$ cross-sectional sample position $[205]$.

All the aforementioned mechanical testing instruments are aligned by optical microscopy, rendering a precision of few micrometers, thus complicating the study of sub-micrometric samples. In the last years, scanning probe microscopes (SPMs) were developed which are compatible with synchrotron beamlines, providing information on surface morphology and allowing for a local characterization of a wide number of physical properties with a resolution going down to the atomic scale. Optical fibers at scanning near-field optical microscopes may be used as detectors for the X-ray excited optical luminescent (XEOL) light $[206,207]$ or the SPM-tip can be used as detector for photoelectrons [208, 209]. Moreover, specific SPMs, such as atomic force microscopes (AFMs), allow for manipulating and modifying matter at the nanoscale. Specially adapted atomic force microscopes were developed at the European Synchrotron (ESRF) in Grenoble (France) for in situ micromechanical testing (X-AFM) [210] and in situ biological studies under physiological conditions [211]. The X-AFM consists of a quartz tuning fork with a resonance frequency of $2^{15} \mathrm{~Hz}=32768 \mathrm{~Hz}$ at which an electrochemically etched tungsten tip is glued on one of its two prongs serving as AFM-tip. The AFM-tip is electrochemically blunted to a radius of curvature in the range of 1-2 $\mu \mathrm{m}$ to get as close as possible to a homogeneous pressure application like for a flat punch. The application of a bias voltage on the tungsten tip allows for collecting photoelectrons excited by the X-ray beam impinging on the sample surface and the tip itself $[208,209,212]$. The recorded photocurrent facilitates the alignment of the AFM-tip with respect to the focused X-ray beam. 
Recently, Ren et al. developed a scanning force microscope for in situ nanofocused X-ray diffraction studies (SFINX) which is combined with coherent X-ray diffraction imaging and Laue microdiffaction for in situ nano-indentation on crystals and for in situ three-point bending tests on nanowires, respectively $[213,214]$. The imaging capabilities of SFINX facilitate the alignment of the SFINX-tip on a selected nanostructure and, thus the study of the physical properties like the mechanical behavior of nano-objects. This is exemplarily demonstrated on Cu nanowires, which were deposited on a Si substrate (Fig. 42(a)). Simultaneously to the imaging of the sample topography by AFM (Fig. 42(b)), the Cu- $\mathrm{K}_{\alpha}$ fluorescence yield (Fig. 42(c)) and the diffraction intensity of the Cu 111 Bragg peak were monitored (Fig. 42(d)) [215]. While the agreement between the topography and the fluorescence map demonstrates a perfect alignment of the AFM-tip and focused X-ray beam with respect to each other, the X-ray diffraction map reveals that only a small part of the nanowire fulfills the Bragg condition showing an imperfect crystallinity of the nanowire. By repeating the in situ imaging at 4 different rocking angles within a range of $1^{\circ}$, the diffraction signal was demonstrated to move by about $5 \mu \mathrm{m}$ along the nanowire indicating torsion of the wire of $0.0035 \mathrm{rad} / \mu \mathrm{m}$ (Fig. 42(e)). This in situ technique thus offers the capability to image the shape of a nanostructure and give simultaneously access to the crystalline structure and deformation with a resolution well below $1^{\circ}$.
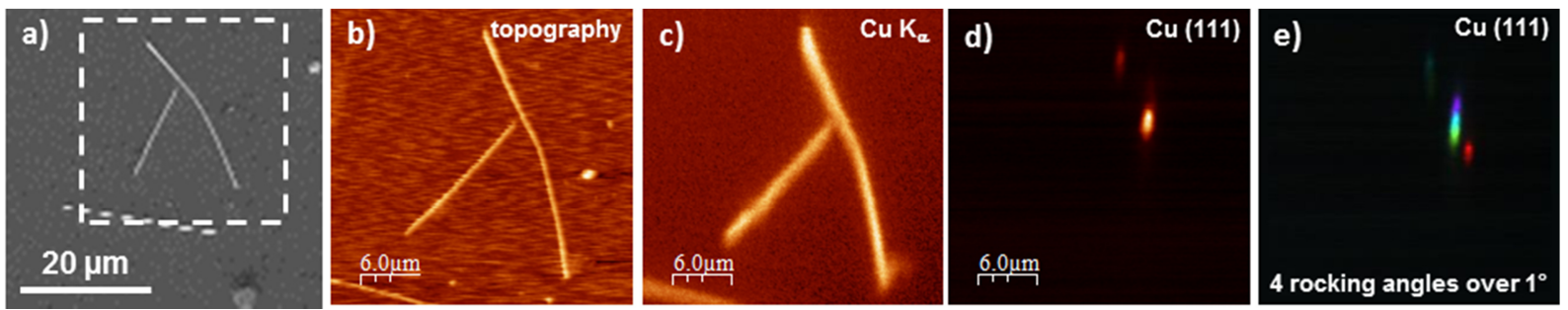

Fig. 42: a) Scanning electron microscopy image of $\mathrm{Cu}$ nanowires deposited on a Si substrate. In situ b) AFM topography image, c) $\mathrm{Cu}-\mathrm{K}_{\alpha}$ fluorescence map, and d) scanning X-ray diffraction map of the Cu111 Bragg peak of the area marked by the dashed square in (a). e) Superposition of four scanning X-ray diffraction maps recorded at different rocking angles within $1^{\circ}$. The different colours represent the diffraction signals for the different rocking angles [215].

\section{III.1 In situ micro- and nano-mechanical tests in combination with Laue microdiffraction}

As introduced above, Laue microdiffraction typically employs a polychromatic X-ray beam that is focused down using a pair of Kirkpatrick-Baez mirrors. While monochromatic X-ray diffraction necessitates the rotation of the sample in order to measure different Bragg peaks, white beam Laue diffraction directly provides a large number of Laue spots that are measured simultaneously without the need of any sample movements. It further gives access to the crystalline structure of a material as well as to the orientation and the deformation of a crystal. However, it provides access to the deviatoric strain in a probed volume only and not to the absolute strain, except in some idealized 
cases. To circumvent this shortcoming and combine mechanical tests with the rainbow filtering technique or an energy dispersive pnCCD is so far not applicable due to long measurement times of several hours, which demand an unreasonably large long-term stability of the testing setup. Regarding plasticity, an elongation of the diffraction spots which is caused by the rotation of the crystalline lattice induced by geometric necessary dislocations stored in the probed volume allows the determination of the slip systems activated during deformation. These features make white beam Laue microdiffraction ideal for studying the mechanical behavior of materials at the microand sub-micrometer length scale.

\section{III.1.i In situ micro-compression tests combined with Laue microdiffraction}

In situ micro-compression studies on FIB milled [4 $\overline{6} 3$ ] oriented Au pillars with diameters of 2 and $10 \mu \mathrm{m}$ were conducted in combination with Laue microdiffraction using the micro-compression device developed at PSI [200]. Stress-strain curves are presented in Fig. 43(a) where the numbers indicate the corresponding Laue diffraction patterns recorded during the in situ mechanical test. By plotting the actual vertical Au crystalline orientation in the inverse pole figure (Fig. 43(b)), the slip systems activated in the $10 \mu \mathrm{m}$ pillar were identified to be the same as the predicted ones, i.e. with the highest Schmid factor. However, for the $2 \mu \mathrm{m}$ diameter pillar, geometrically unexpected slip systems were found. The path of the satellite peak on the detector (from the deformed part of the micro-pillar) revealed that the unexpected rotations were composed of several activated slip systems (Fig. 43(c)), which was further confirmed by slip traces observed by SEM imaging. This unexpected behavior was attributed to strain gradients present in the pristine pillar, which was inferred from the asymmetric peak shape (Fig. 43(d)).

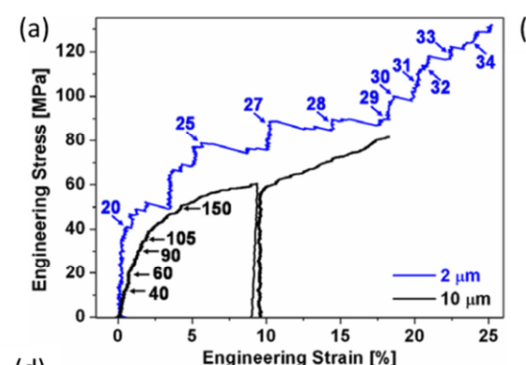

(d)

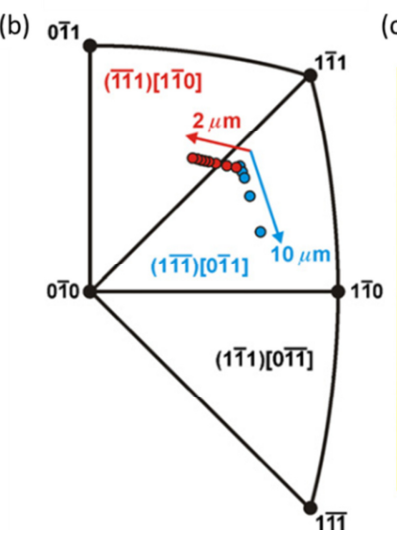

(c)

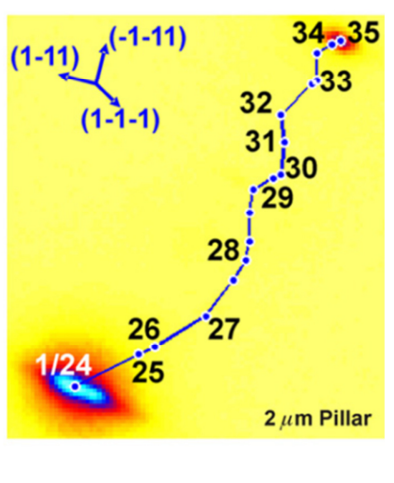

Fig. 43: a) Stress-strain curves for a FIB milled Au micro-pillar during compression [200]. The arrows indicate the corresponding Laue microdiffraction pattern recorded during the mechanical test. b) Inverse pole figure showing the evolution of the actual vertical crystalline axis during the compression test [216]. c) Path of the satellite peak of the $\mathrm{Au} \overline{\mathbf{2}} \mathbf{} 0 \mathrm{Laue}$ spot on the detector with the same numbering as marked in (a) [216]. d) Intensity distribution of the $\overline{2} 2 \overline{2}$ Laue peak at a load of 0 MPa (\# 0), 40 MPa (\# 20), and 77 MPa (\# 25), respectively [200]. 
In later experiments, in situ compression tests on FIB milled micro-pillars of different FCC metals with diameters ranging from 2 to 4 ? $\mathrm{m}$ were performed [217]. While at the beginning of the loading an unexpected slip system is evidenced by the sample rotation, the rotation changes to the predicted direction for large deformations. The authors defined the stress at which the rotation direction changes as the "Laue yield stress" which was found to be less sensitive to the size. They thus attributed the size dependent yield strength reported in literature to the strong size-dependent strain hardening in FIB milled microstructures, i.e. the evolution of the microstructure during deformation and not to a size dependence of the initial strength of the pillars. Similar microcompression tests on tungsten pillars, which have a BCC lattice structure, were reported by Marichal et al. [218]. From the obtained diffraction patterns, it was concluded that the slip planes in this BCC material are $\{110\}$ planes and the usually observed $\{112\}$ slip traces are composed of several simultaneously activated slips with $\{110\}$ planes.

During micro-compression tests, friction between the indenter flat punch and the top end of the micro-pillar hinders the lateral movement of the structure and induces additional constraints which generate a bending component. Thus, strain gradients are created and the central portion of the crystal rotates so that both the slip planes and the slip direction approach to or moves away from the loading axis. The effect of the constraints has been investigated in detail in a series of studies by Kirchlechner et al. [202, 219, 220] and recently reviewed by Robach et al. [167]. The inverse pole figure displayed in Fig. 44(a) shows the actual vertical crystalline direction during in situ compressions of square Cu pillars with a side length of $L=7 \mu \mathrm{m}$ and an aspect ratio of height to lateral length $h / L=$ 3:1 $[167,219]$. During the compression tests, the X-ray beam was focused to the center of the pillars. The rotation of the center of pillar B was found to deviate from the expected direction. However, correlating this rotation to the stress-stain curve of this pillar (see Fig. 44(b)) revealed that for strains $<5 \%$ almost no rotation can be observed. Thus, at the beginning of the loading the lateral motion of the top end is free while for strains $>5 \%$ the rotation indicated additional constraints. Upon reloading, the lateral constraints are temporally removed and were re-activated only when the strain state exceeded $18 \%$. Post-mortem raster scanning studies of the unloaded pillar (see Fig. 44 (c)) revealed that the strain gradients generated by the lateral constraints were mostly accommodated by the storage of geometrically necessary dislocations near the ends of the pillar. 

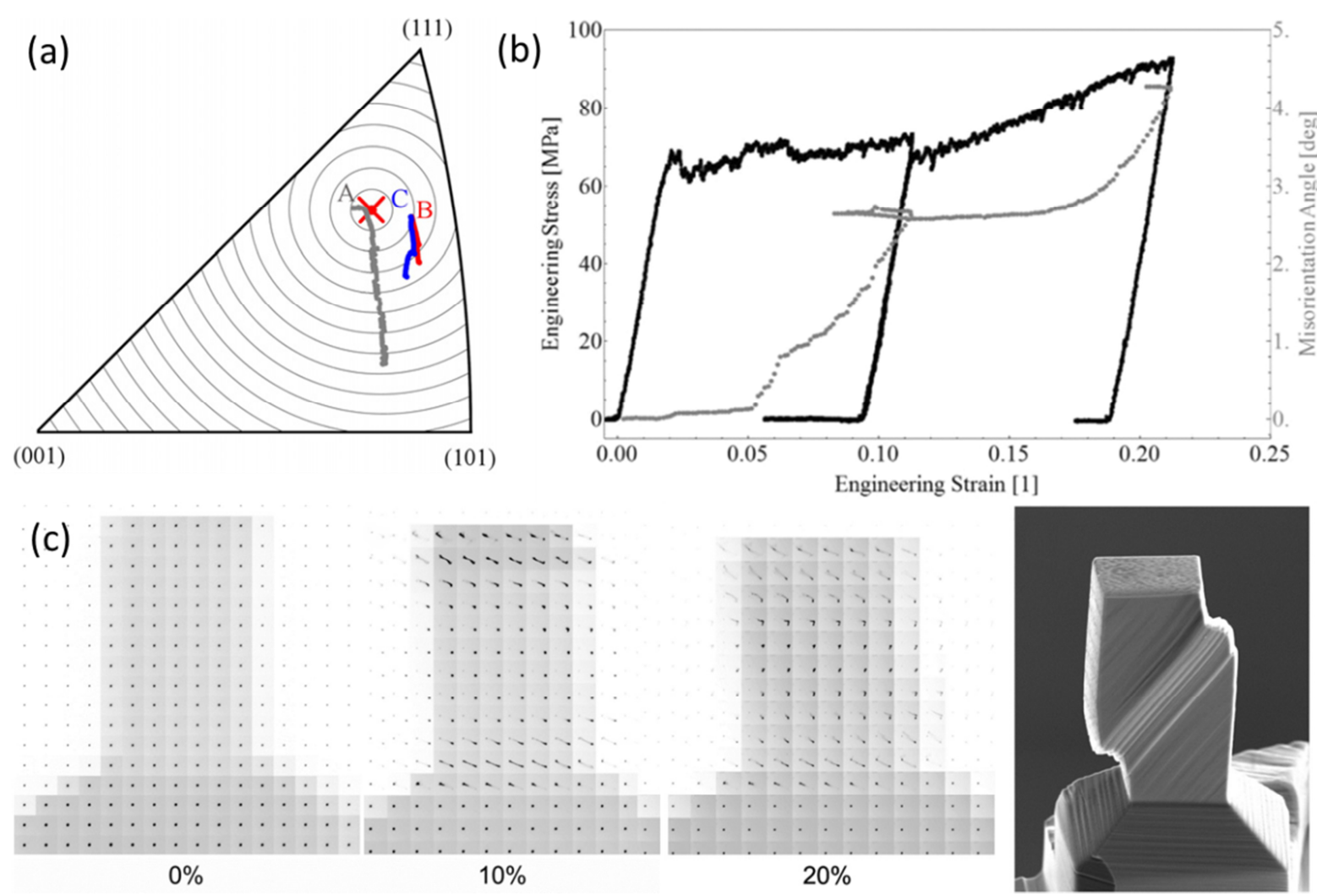

Fig. 44: a) Inverse pole figure showing the actual vertical crystalline directions of three FIB milled [234] oriented Ni pillars A, B, and C during in situ compression. b) Stress-strain curves for pillar B correlated with the misorientation measured in the pillar center. c) Maps of the Ni 040 Laue spot at $0 \%$, at $10 \%$, and at $20 \%$ strain for pillar B and SEM image of the pillar after deformation [167, 219].

These studies demonstrate the power of in situ Laue microdiffraction to elucidate the mechanical behavior at the microscale, which is not accessible by other means and uncovering unexpected slip systems. This technique allows for studying samples that are much thicker than electron transparent specimens for TEM examinations while it still enables looking inside the material and to monitoring the elastic and plastic deformation. This is in clear in contrast with SEM which yields only surface information. By further combining the in situ Laue microdiffraction method with the differential aperture $\mathrm{X}$-ray microscopy (DAXM) technique, three-dimensional information of mechanically deformed structures may become accessible.

The studies reported above exclusively focused on specimen prepared by FIB milling that may induce defects, thus altering the mechanical behavior of the structure under investigation, in particular for sub-micrometric samples where the surface-to-volume ratio becomes more and more important. In addition, the imperfect alignment of the indenter head with respect to the structure under investigation by optical microscopy as well as the force sensitivity of the testing apparatus hamper proper examination of nanostructures. 


\section{III.1.ii In situ nanowire bending tests combined with Laue microdiffraction}

The aforementioned complications were resolved by the development of the in situ AFM "SFINX" that facilitates the alignment of the indenter, i.e. the SFINX-tip, with respect to a nanostructure by AFM imaging of the sample topography. Moreover, the FIB milling process was circumvented by using as-grown metal nanowires ( $200 \mathrm{~nm}$ in diameter, $>10 \mu \mathrm{m}$ in length) that were prepared by vapor phase deposition and placed across Si micro-trenches, thus facilitating threepoint bending tests in combination with Laue microdiffraction at the BM32 beamline at ESRF [214]. A sequence of Laue microdiffraction patterns showing the Si 004 and the Au 111 Laue spot of the Si substrate and the Au nanowire, respectively, is displayed in Fig. 45(a). For the pristine nanowire the two Laue spots are superimposed. With increasing load the Au 111 Laue spot is displaced on the detector and moves away from the Si 004 substrate peak. During unloading, the Au Laue spot returns towards its original position. After complete unloading the two Laue spots are again superimposed indicating a fully elastic deformation. From the displacement and the positions of the Laue spots on the detector, the rotation of the Au nanowire around three distinct crystallographic orientations was calculated as presented in Fig. 45(b). Although the applied force was not directly determined in this experiment, the mechanical behavior of the nanowire was "reconstructed" by finite element method (FEM) simulations revealing that geometric non-linearities play an important role in three-point bending tests and that the maximum stress applied was $450 \mathrm{MPa}$.
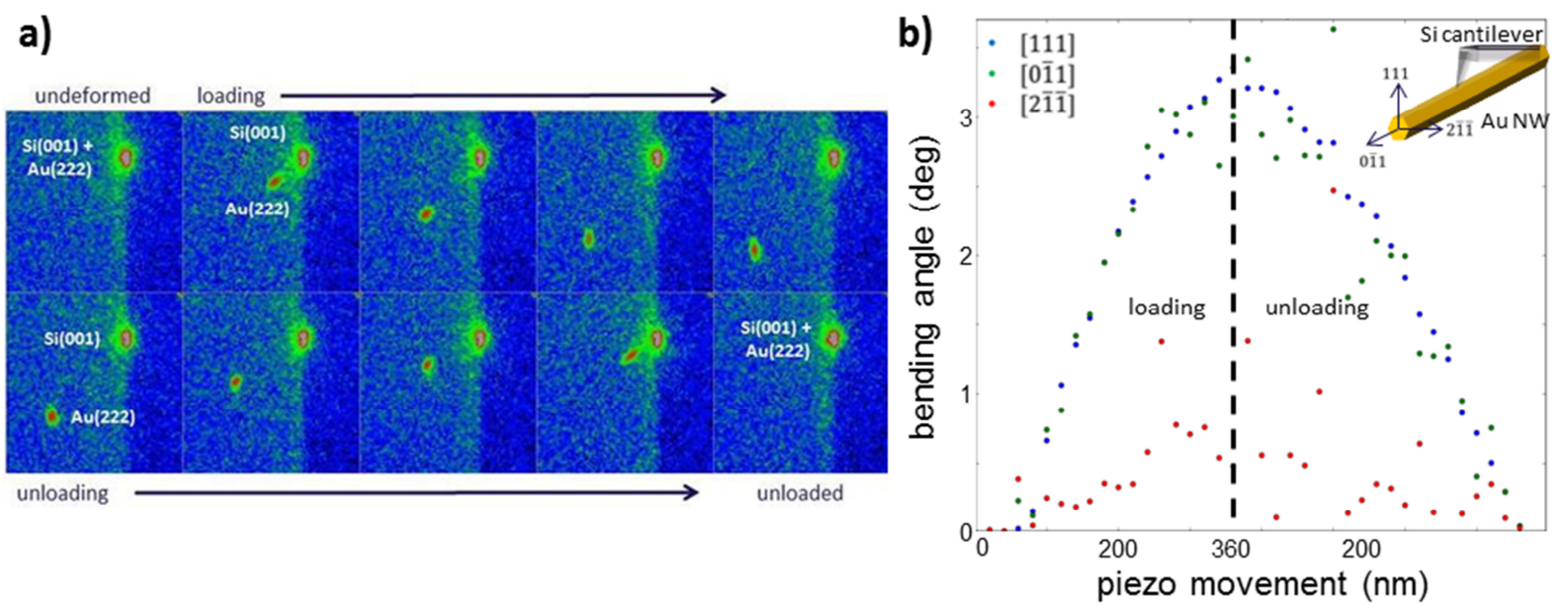

Fig. 45: a) Sequence of Laue microdiffraction patterns showing the evolution of the Si001 and the Au222 Laue spot during three-point bending of a Au nanowire placed across a Si micro-trench. b) Bending angles for the $A u$ [111], the $A u[0 \overline{1} 1]$, and the $A u$ [2 $\overline{1} \overline{1}]$ directions (corresponding to the loading direction, the nanowire growth axis, and the direction perpendicular to the loading plane) inferred from the in situ Laue microdiffraction patterns as a function of the movement of the piezostage carrying the AFM cantilever [214].

As in the aforementioned experiment, in situ micro- and nano-mechanical tests using submicrometer focused X-ray beams typically concentrate on one single position in an extended object 
due to the fact that any movement of diffractometer motors can induce vibrations which eventually lead to the damaging of the nano-object under investigation. In order to circumvent this restriction, Leclere et al. recently demonstrated a novel beam scanning technique which allows for mapping a sample in two dimensions during mechanical deformation [221]. This technique gives access to the profile of the complete nanowire under load as well as to its boundary conditions, i.e. whether the wire is thoroughly clamped or simply supported. This beam scanning approach bases on the lateral and/or angular displacement of the Kirkpatrick-Baez mirrors used for focusing the incident white Xray beam and, thus displacing the $\mathrm{X}$-ray beam focus on the sample surface.

A scanning electron micrograph of a self-suspended Au nanowire, which was loaded using the AFM-tip, is displayed in Fig. 46(a). The evolution of the Au 222 spot along the nanowire in the pristine state as well as for three different deformation states is presented in Fig. 46(b). For the pristine nanowire the Laue spot remains at its position on the detector, whereas it describes a sinusoidal behavior for the deformed states with increasing amplitude for higher applied force. From the displacements of the Laue spots on the detector the bending angles along the nanowire were inferred (see Fig. 46(c)) also revealing a sinusoidal behavior. It remains close to $0^{\circ}$ at the clamping of the self-suspended nanowire as well as at the loading point, while the maximal bending is located about half way between the loading point and the supports. The authors computed the complete nanowire profiles from the bending angles (Fig. 46(d)) showing a maximal deflection of $300 \mathrm{~nm}$ in the nanowire center.

This new X-ray beam scanning technique offers unique advantages for micro- and nanomechanical tests on extended objects facilitating to probe the whole micro- or nanostructure under load and, consequently gaining access to the elastic and plastic deformation of the whole object instead of probing only one singular position. 


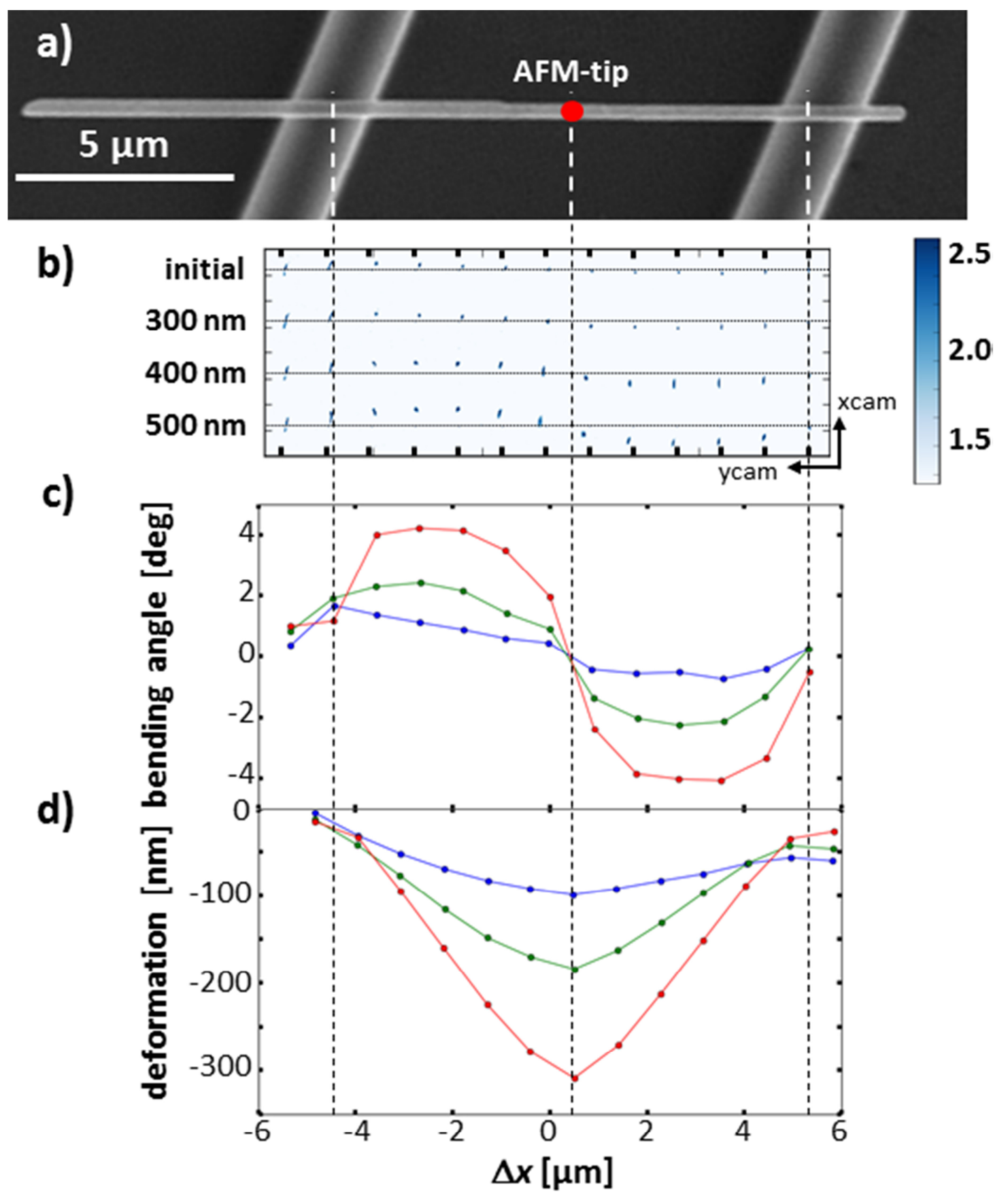

Fig. 46: a) Scanning electron microscopy image of a self-suspended Au nanowire crossing a $10 \mu \mathrm{m}$ wide $\mathrm{Si}$ micro-trench. b) Sequences of the Au222 Laue spot along the nanowire during three-point bending tests for different loads. c) Bending angle along the nanowire calculated from the orientation matrices UB which were inferred from the position and displacement of at least eight Au Laue spots on the detector. d) Deformation along the nanowire for the different deformation stages computed from the bending angles shown in part (c) [221].

The plasticity of Au nanowires was investigated ex situ as well as in situ using Laue microdiffraction [153]. A scanning electron micrograph of a mechanically deformed Au nanowire is presented in Fig. 47 together with four Laue microdiffraction patterns taken along the deformed 
nanostructure. While at the reference position at the left hand side of the nanowire, where it was not loaded previously, the Laue spots are well-defined, the diffraction peaks are elongated in the zone of mechanical deformation. This streaking and in particular the direction of the streaking indicate the storage of $[01 \overline{1}](111)$ type of geometrically necessary dislocations. The fact that the streaking decreases along the nanowire while approaching the kink clearly visible in the SEM image implies a decrease in the density of stored GNDs. The red lines in the insets represent the theoretical positions of the Laue spots including the streaking induced by the stored GNDs. The deviation of the red lines from the experimental Laue spots in the contrary indicates an additional residual torsion of the plastically deformed Au nanowire.

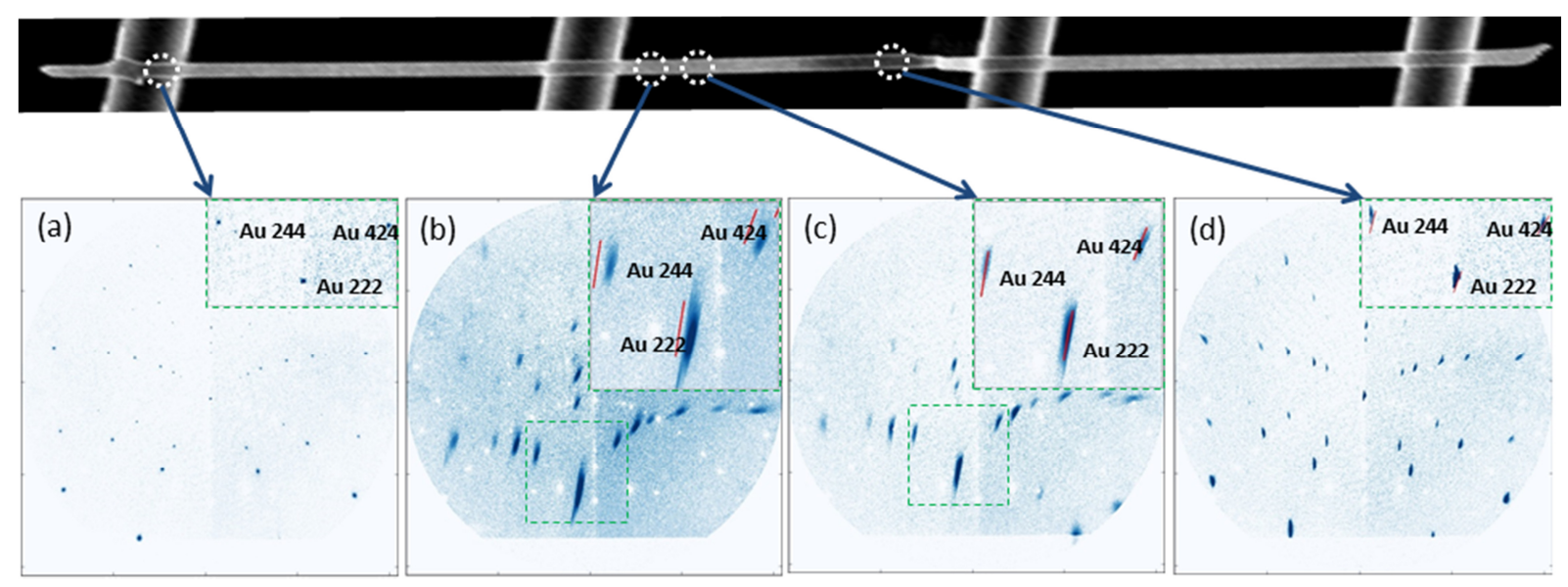

Fig. 47: Scanning electron micrograph of a gold nanowire mechanically deformed in a three-point bending configuration and four Laue microdiffraction patterns recorded along the deformed structure. The red lines in the inset represent the theoretical position of the Laue spots and the streaking direction for the storage of $[01 \overline{1}](111)$ type of dislocations.

As demonstrated in this section, Laue microdiffraction coupled with dedicated mechanical testing devices largely facilitates in situ studies of the elastic and plastic behavior of micro- and nanostructures. The streaking of the Laue spots is a clear signature of geometrically necessary dislocations stored in the material and the streaking direction allows for determining the type of dislocations. However, while Laue microdiffraction largely simplifies the experiment by the fact that the sample does not have to be rotated during mechanical loading, the determination of the spherical strain (which represents only volumetric deformation and is an isotropic tensor) is not straight forward but necessitates the measurement of the energy for at least one Laue spot. In the following section, works with monochromatic X-ray diffraction will be reviewed that provides direct access to the full strain tensor. 


\section{III.2 In situ micro-mechanical testing and focused monochromatic X-ray diffraction}

$\mathrm{X}$-ray nanodiffraction on a lamella of an indented $\mathrm{CrN}-\mathrm{Cr}$ multilayer thin film in a transmission geometry providing access to the local stress fields around the indenter imprint was demonstrated by Stefenelli et al. [222]. This diffraction technique was later on coupled with the in situ indenter at the P03 beamline at PETRA III for monitoring the strain field and the microstructural changes in Zrbased bulk metallic glasses [204] and in several micrometer thick TiN films (Fig. 48) [205] during the indentation process. In the latter case, the authors were able to follow the tilting of TiN crystallites, to determine the strain and strain gradients from the peak positions and the peaks widths and they further related the experimental findings with crack propagation in the film which is influenced by interfaces within the film itself and with the substrate. Thus, in combination with in situ mechanical loading, this diffraction technique makes accessible the complete elastic strain field of a material under load.

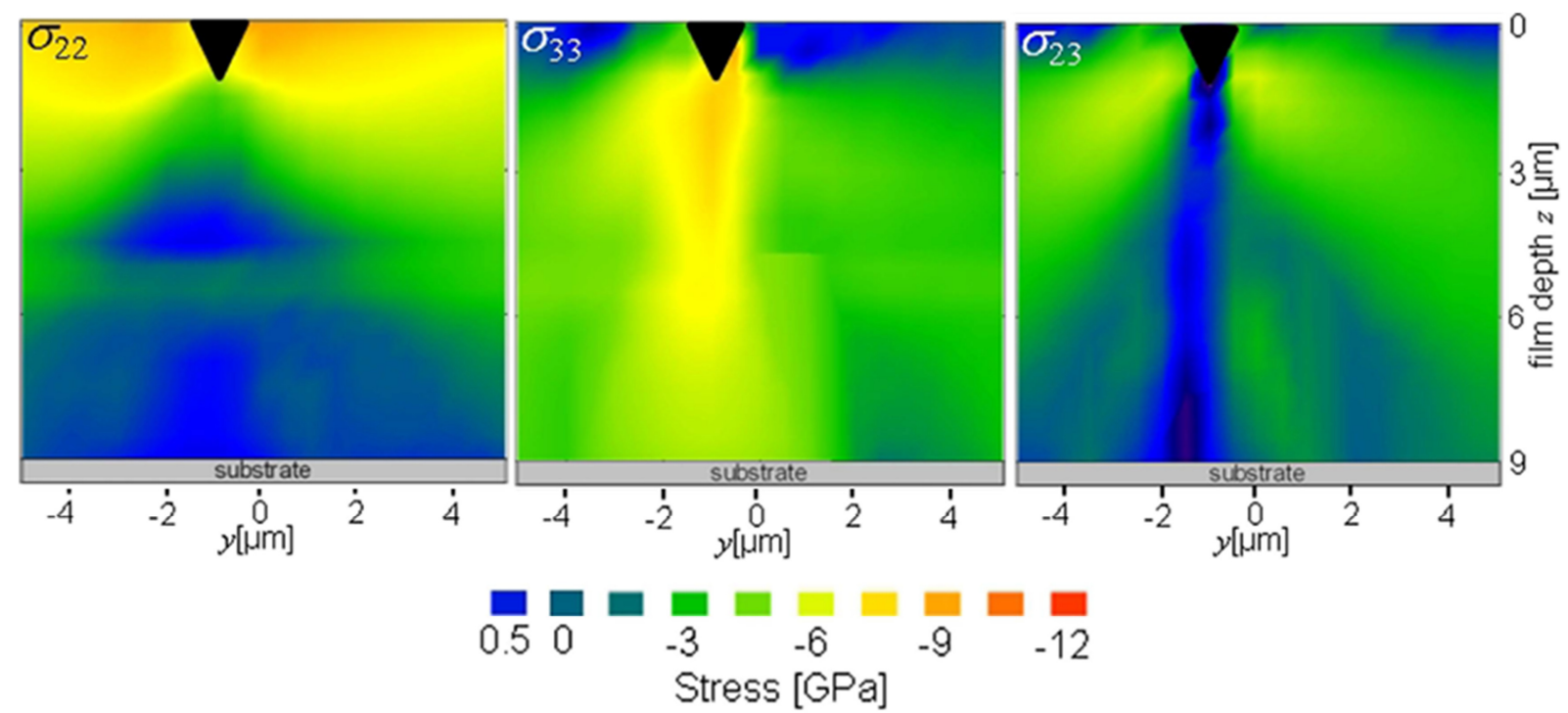

Fig. 48: Stress distribution $\sigma_{22}, \sigma_{23}$, and $\sigma_{33}$ in a $9 \mu \mathrm{m}$ thick TiN film indented with a load of 1.4 N [205].

The aforementioned in situ technique facilitates the measurement of the strain field during nano-indentation. However, it does not allow for investigating an individual micro- or nanostructure. The X-AFM, on the contrary, that was developed at the European Synchrotron ESRF in Grenoble (France) renders possible the compression of a single microstructure and monitoring the deformation by monochromatic X-ray diffraction in situ as demonstrated on a single SiGe island (see Fig. 49(a)) $[210,223]$. For pressure application, the AFM-tip was brought into contact with the selected structure and then moved stepwise down (Fig. 49(b)). At each step a 2D X-ray diffraction pattern in the vicinity of the Si 004 Bragg peak (Fig. 49(c)) as well as the resonance frequency of the 
tuning fork was recorded simultaneously. With increasing load the CTRs of the SiGe island side facets collapse (II and III) and the Bragg signal of the SiGe island moves to larger $q_{z}$ values (IV and V) until it is superimposed with the Bragg reflection of the Si substrate (VI). At the same time, the resonance frequency of the tuning fork shifted to higher frequencies from about $29 \mathrm{kHz}$ for the free tuning fork

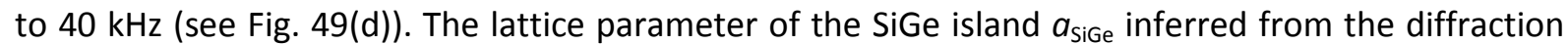
data as well as the tuning fork resonance frequency $f_{\text {res }}$ are shown as a function of the travel $\Delta z$ of the sample against the AFM tip in Fig. 49(e) revealing a direct correlation between the tuning fork resonance frequency and the SiGe island lattice parameter. The authors approximated the contact of the AFM-tip with the top facet of the island using a Hertzian contact model [61]. The stiffness of the contact area was inferred from the frequency shift of the tuning fork. This approach yielded a Young's modulus of (108 \pm 12$) \mathrm{GPa}$, which is very close to the literature value for a SiGe island of 105 GPa.

a)

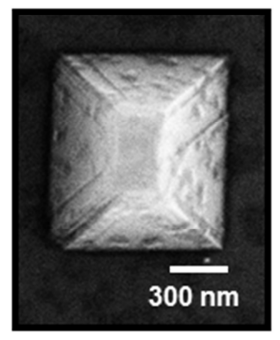

b)

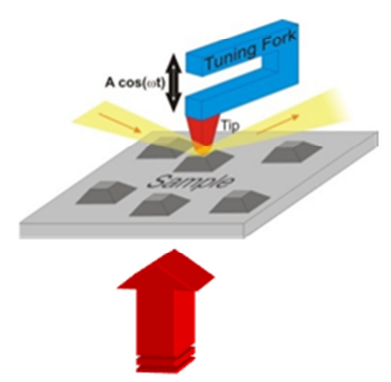

d)

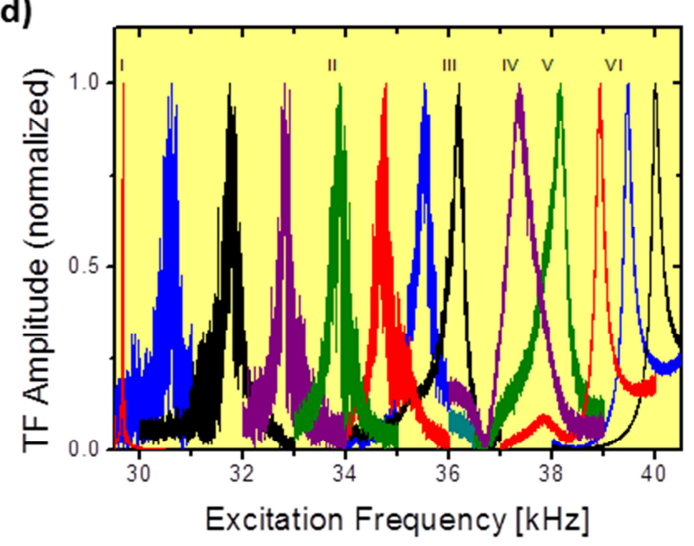

c)
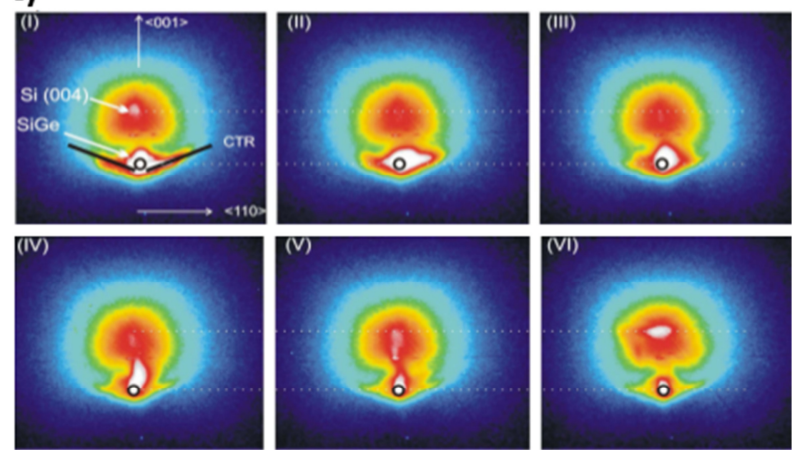

e)

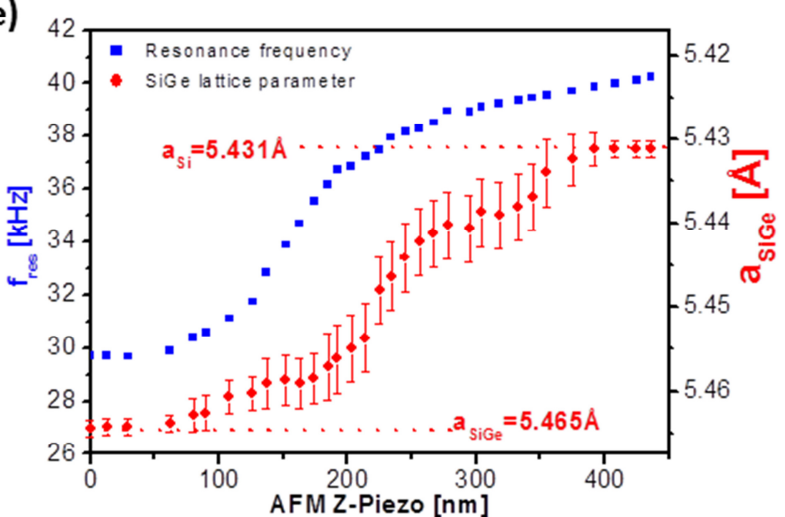

Fig. 49: a) Scanning electron micrograph of a SiGe island. b) Schematic illustration of the mechanical loading of a SiGe island using the AFM-tip and simultaneous X-ray diffraction. c) X-ray diffraction patterns for 6 consecutive pressures applied on an individual SiGe island. The Si (004) and SiGe Bragg reflections are marked by arrows while the CTRs originating from the island side facets are highlighted by black lines, and the substrate CTR is encircled. d) Normalized resonance frequencies of the AFM tuning fork. The resonance curves corresponding to the diffraction maps from panel (c) are indicated by Roman numerals I to VI. (e) Variation of the SiGe island lattice parameter (red) and the tuning fork resonance frequency as a function of the sample displacement $\Delta z$. Taken from refs. [210] and [223]. 
It should be noted that 2D X-ray diffraction images represent only one cut through reciprocal space while Bragg reflections of micrometer and sub-micrometer structures are three-dimensional. It is thus indispensable to record the three-dimensional intensity distribution in the vicinity of a Bragg reflection in situ. Such 3D reciprocal space maps are typically recorded by rocking the sample, thus varying the angle of incidence of the X-ray beam. However, every movement of diffractometer motors may induce vibrations which may eventually lead to the damaging of the structure under mechanical load. It is therefore desirable to record 3D-RSMs by other techniques than by ordinary rocking scans. Cornelius et al. developed an approach where the energy of the incident X-ray beam is scanned facilitating to probe the three-dimensional intensity distribution in the vicinity of a Bragg peak without moving any diffractometer motor [224]. However, the chromaticity, i.e. the dependence of the focal distance of the employed focusing optics on the energy of the incident $X$ rays, strongly limits the scan range when not adjusting the distance between the focusing optics and the sample position. This energy scanning approach was then successfully demonstrated on a single SiGe island that was compressed using the X-AFM [225].

Two-dimensional diffraction patterns, 3D reciprocal space maps measured by the energy tuning approach, and $\left(q_{\mathrm{x}}, q_{\mathrm{z}}\right)$ cuts through the 3D-RSMs at $q_{\mathrm{y}}=0$ for a SiGe island at different deformation stages are displayed in Fig. 50 in the upper, central, and lower line, respectively. The positions of the Si 004 substrate Bragg peak at $q_{\mathrm{z}}=46.3 \mathrm{~nm}^{-1}$ and of the SiGe island at $q_{\mathrm{z}}=46.05 \mathrm{~nm}^{-1}$ are indicated by dashed lines and the crystal truncation rods are highlighted by dotted lines. During mechanical loading the central part of the diffraction signal of the SiGe island disappears (see Fig. 50(b) and (c)). The fact that at the same time the Si 004 Bragg reflection becomes more diffuse and develops a substructure indicates that the missing island signal becomes superimposed with the substrate Bragg peak and strain is induced in the substrate. Moreover, the facet streaks turn to steeper angles with increasing load implying that the island shape is being deformed. At highest loading (see Fig. 50(d)) the CTR angle returns back to its original position accompanied by a vanishing island signal and the appearance of speckles which may indicate the presence of defects or a fracture of the central island part. Finite element method simulations revealed that a stress of 2 to $3 \mathrm{GPa}$ was applied at highest load. 

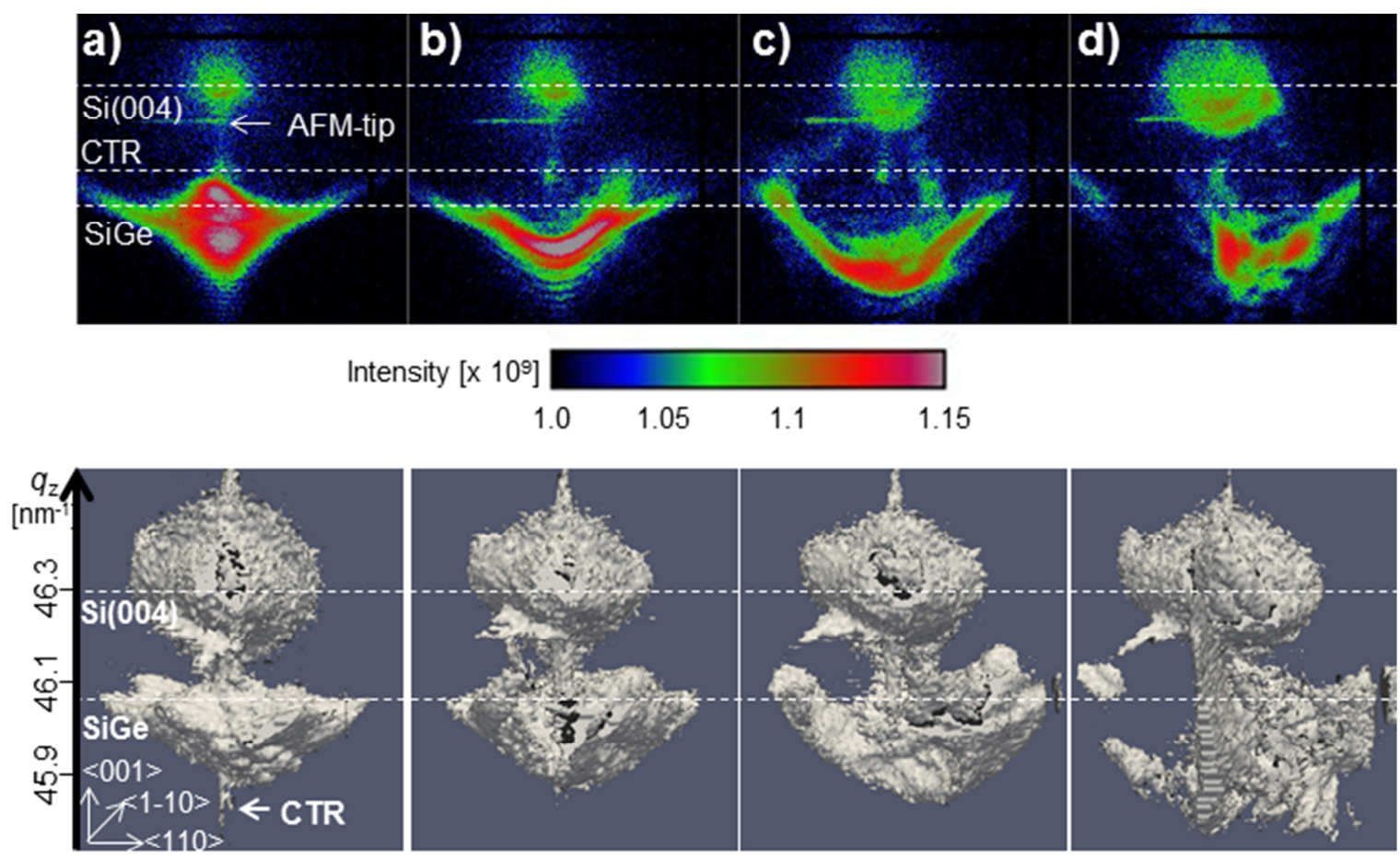

Isosurface at intensity of $1.1 \times 10^{9}$
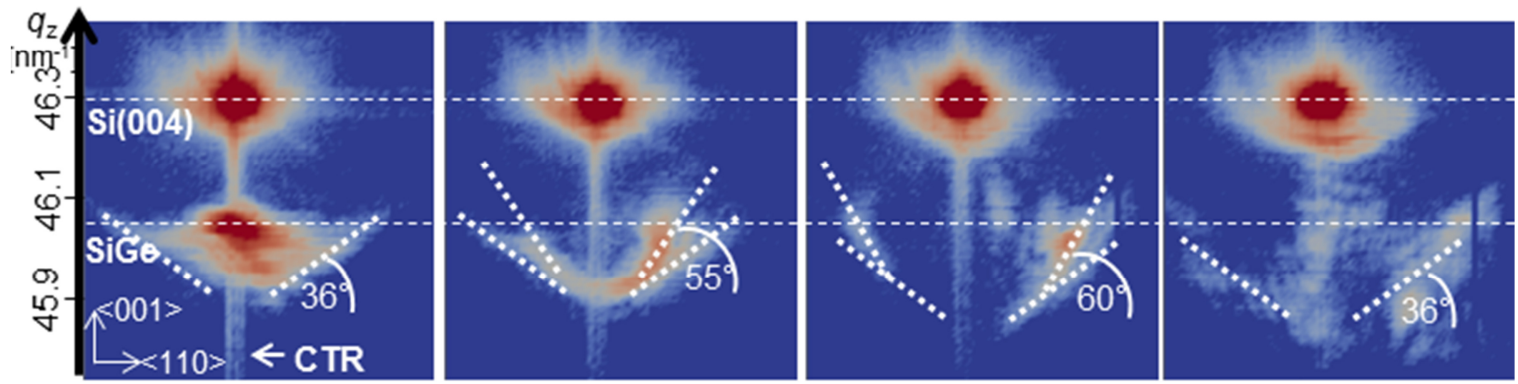

Intensity $\left[\mathrm{x} 10^{\circ}\right]$

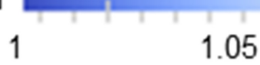

1.1

1.15

Fig. 50: The 2D X-ray diffraction patterns (upper row), reconstructed 3D reciprocal space maps (central row), and $\left(q_{\mathrm{x}}, q_{\mathrm{z}}\right)$ cuts through the in situ 3D-RSMs at central $q_{\mathrm{y}}$ (lower row) for (a) a pristine SiGe island and (b)-(d) the same SiGe island at different deformation stages. The position of the Si (004) Bragg reflection and the signal of the $\mathrm{SiGe}$ island is indicated by dashed lines. The dotted lines highlight the CTRs originating from the island side facets [225].

\section{III.3 In situ Bragg coherent diffraction imaging}

Monochromatic X-ray diffraction using partially coherent X-ray beams as demonstrated in the previous section allows for mapping the strain field within a sample with a resolution given by the beam size and monitoring shape changes. To gain access to the displacement field inside a specimen with a resolution in the picometer range and with a spatial resolution far better than the actual beam size, fully coherent X-ray beams are necessary rendering possible Bragg coherent diffraction imaging. The use of phase retrieval algorithms then allow for reconstructing the shape as well as the phase within the specimen providing access to the displacement field. 
Three-dimensional coherent X-ray diffraction was further used to study the mechanical behavior of a single grain within a polycrystalline Au thin film that was deposited on a Si substrate [226]. Here, mechanical load was applied via thermal cycling between room temperature and $250{ }^{\circ} \mathrm{C}$ leading to different thermal expansions of the gold thin film and the Si substrate, thus imposing mechanical deformation. These experiments evidenced a large $u_{z}$ in-plane anisotropy which decreased during heating and which was attributed to inter-grain interactions. Very recently, Yau et al. demonstrated grain Bragg coherent diffraction imaging (gBCDI) on a polycrystalline gold thin film that was heated resolving grain boundary and dislocation dynamics in individual grains in three dimensions with a spatial resolution of $10 \mathrm{~nm}$ and sub-angstrom displacement field resolution [227]. The reconstructed image, a cross-sectional view, and a cross-section of the reconstructed displacement field for an individual gold grain are presented in Fig. 51(a), (b), and (c). The black arrow indicates the [111] scattering vector while the white arrow highlights a low-amplitude region in the Bragg electron density and a corresponding discontinuity in the displacement field being consistent with a dislocation. Fig. 51(d), (e), and (f) show the corresponding results for the same grain at $400{ }^{\circ} \mathrm{C}$ evidencing grain growth near the dislocation and the absence of the dislocation itself.

\section{As-synthesized State}
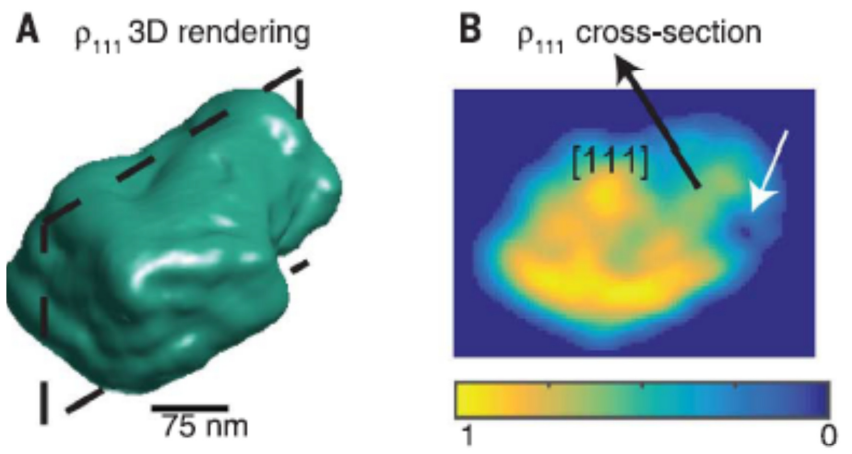

C $u_{111}$ cross-section

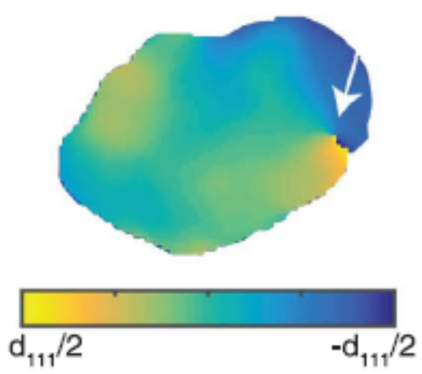

$400{ }^{\circ} \mathrm{C}$ State

D $\rho_{111} 3 D$ rendering

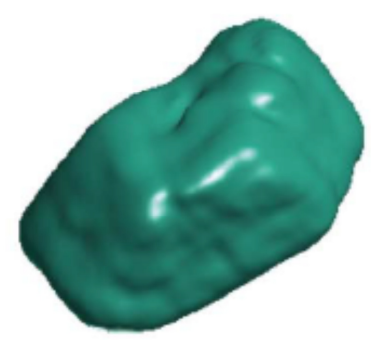

E $\rho_{111}$ cross-section

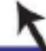

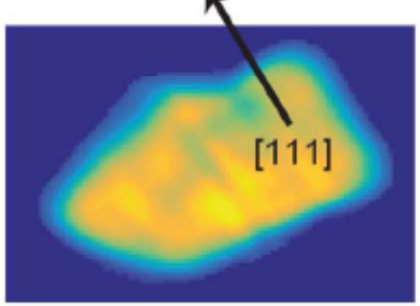

F $\mathrm{u}_{111}$ cross-section

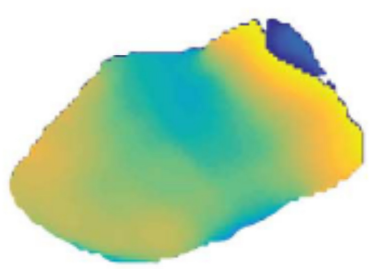

Fig. 51: Grain boundary and dislocation movement in a single gold grain within a polycrystalline Au thin film during heating. a) 3D rendering of the reconstructed grain image, b) cross-section view (cross-section location shown by black dashed line in (a)] of the reconstructed Bragg electron density, and c) crosssection view of the reconstructed displacement field for the as-synthesized state. The black arrow indicates the [111] scattering vector while the white arrow highlights a low-amplitude region in the Bragg electron density and a corresponding discontinuity in the displacement field being consistent with a dislocation (d f) The same as in (a) to (c) but at $400^{\circ} \mathrm{C}$ [227]. 
Although Yau et al. performed in situ BCDI measurements during heat treatment in a polycrystalline environment, a limitation of this and prior BCDI measurements is that only a single Bragg peak from an essentially randomly chosen grain is studied. To connect with emergent behaviors, it will be necessary to study clusters of neighboring grains. Recent developments of "darkfield X-ray microscopy" [42] offers an opportunity to "zoom" from the mesoscale down to imaging of defect motions and behaviors in larger-sized grains than are easily accessible to BCDI. Although not directly measuring angstrom-scale atomic displacements seen in BCDI, this technique offers the possibility of correlating responses on different length scales in the same sample [228].

Actual in situ nano-mechanical tests combined with BCDI which do not rely on thermal treatment and different thermal expansion coefficients of sample and substrate but on mechanical loading of an individual nanostructure were recently reported by Dupraz et al. $[229,230]$ and Shin et al. [231] demonstrating the in-situ nano-indentation of a Au crystal and in-situ tensile testing of a single Au nanowire, respectively. A scanning electron micrograph of a typical Au crystal used for nano-indentation is presented in Fig. 52(a) with the black lines amplifying the various facets of the crystal. The inset schematically illustrates the experimental setup combining nano-indentation and coherent X-ray diffraction. The $q_{\mathrm{y}}-q_{\mathrm{z}}$ cut through the 3D reciprocal space map of the Au 111 Bragg peak is displayed in Fig. 52(b). The very weak distortion of the Bragg peak suggests a very low amount of residual strain in the crystal which was prepared by dewetting of a gold thin film on a sapphire substrate. The main source of strain in these crystals is the thermoelastic strain caused by different thermal expansion coefficients of the sapphire substrate and gold. The electron density reconstructed from the 3D coherent X-ray diffraction pattern is presented in Fig. 52(c) demonstrating a perfect agreement between the SEM image and the reconstructed shape of the pristine crystal. The crystal exhibits a Winterbottom equilibrium shape where all the $\{100\}$ and $\{111\}$ facets connected with rounded interfaces are clearly identified from the reconstructed electron density.
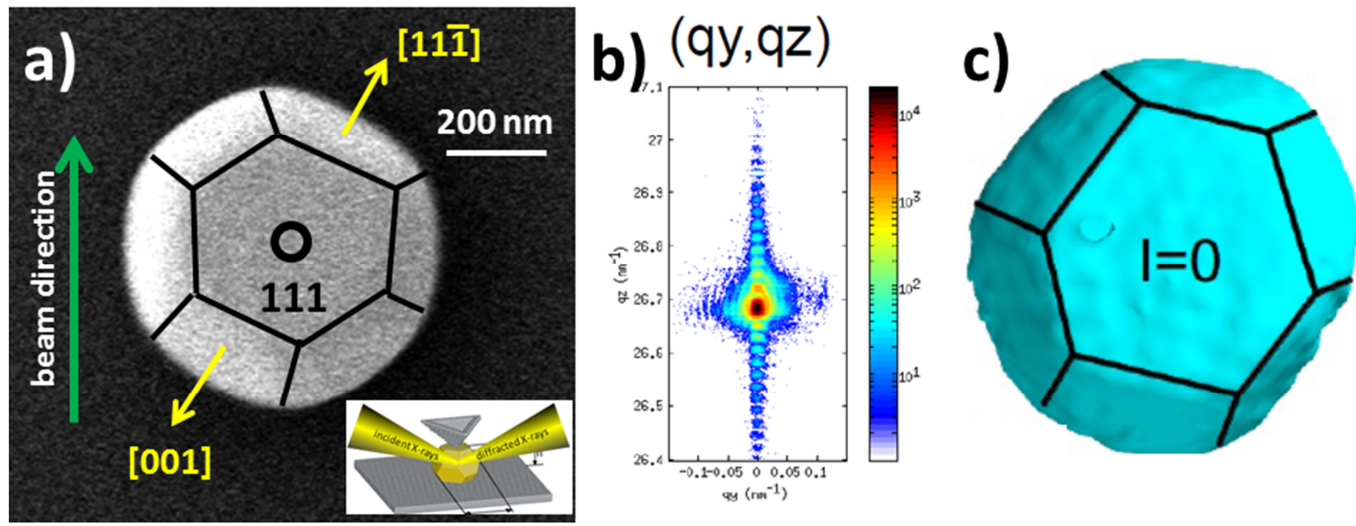

Fig. 52: a) SEM image of Au nano-crystal prepared by dewetting of a 45-nm Au thin film on a sapphire substrate. The inset illustrates the in-situ indentation and the green arrow represents the direction of the incident X-ray beam. The facets are highlighted by black solid lines. b) Cut through a 3D coherent X-ray 
diffraction pattern and (c) reconstructed electron density of the Au nanocrystal shown in panel (a) [229Erreur ! Signet non défini.].

The Au nano-crystal was indented using SFINX and 2D coherent X-ray diffraction patterns were recorded simultaneously. After persistent changes were observed in the diffraction patterns, the Au crystal was completely unloaded and a Bragg coherent X-ray diffraction pattern was recorded. Here, the reconstructed electron density reveals a loop-shaped feature with a diameter of $\sim 30 \mathrm{~nm}$ in the center of the particle about $100 \mathrm{~nm}$ above the particle-substrate interface. This strongly resembles intensity drops which are expected in the vicinity of dislocations. The (110), the (112), and the (111) planes displayed in Fig. 53(b), (c), and (d) intercept the loop in two locations where a pair of vortices with opposite directions is observed. The profile of the $u_{111}$ displacement field around the defect is very similar to the $u_{111}$ displacement field around simulated prismatic dislocation loops [232]. Between the two phase vortices, the rapid phase variations along the loop axis are localized to a region which does not exceed the loop diameter. In the plane that intercepts the loop perpendicularly to its axis, the phase shift between the regions inside and outside the loop is roughly equal to $\pi$ coinciding with simulations.

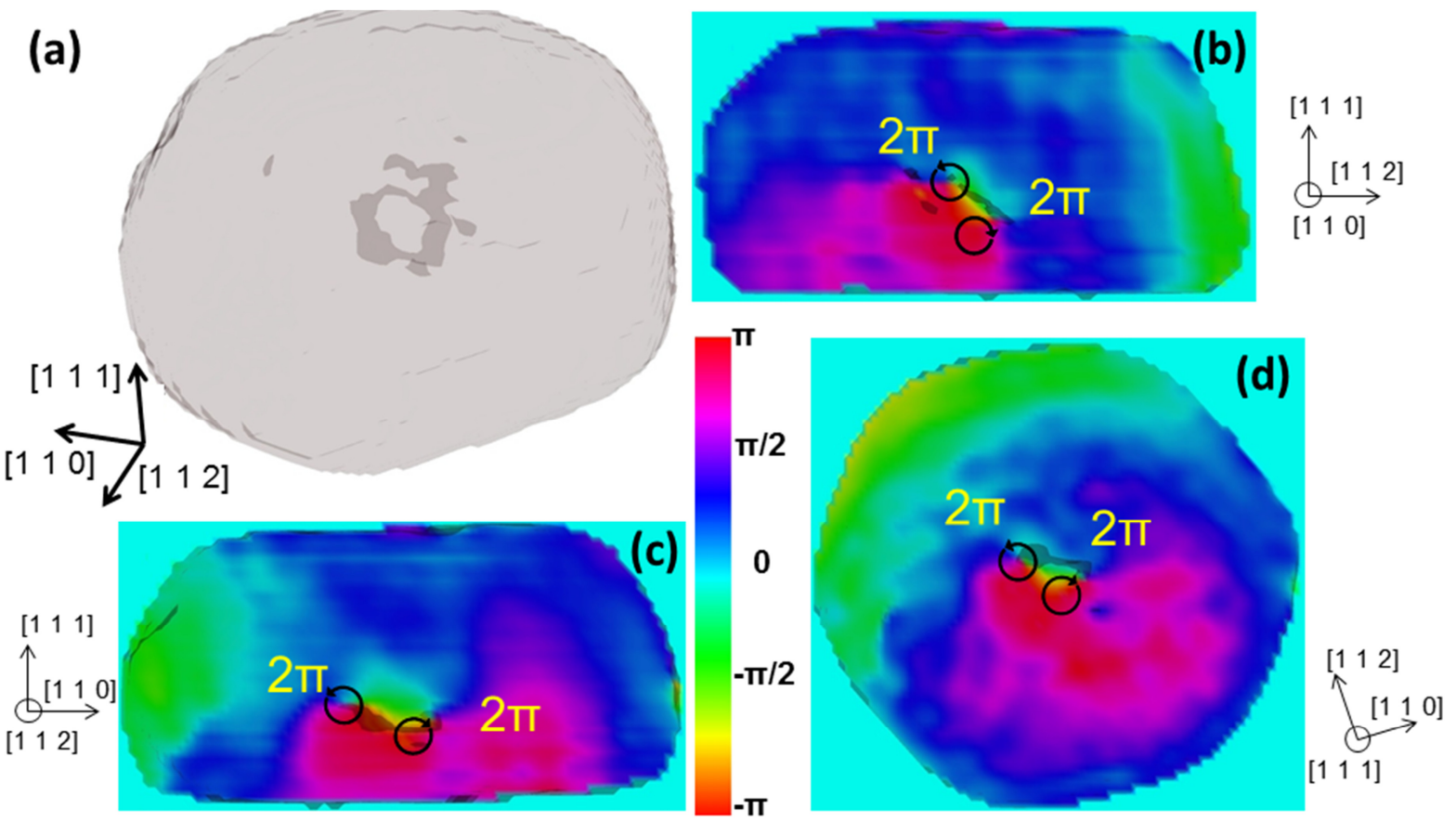

Fig. 53: (a) Reconstructed electron density drawn at 15\% of the maximum value. The drop in the electron density indicates the position of the dislocation loop. Reconstructed $u_{111}$ displacement field in (b) the (110), (c) the (11-2), and (d) the (111) planes which intercept the loop in two locations [229].

This work demonstrated for the first time the semi in situ combination of mechanical loading and BCDI of a sub-micrometer object and the imaging of a mechanically induced prismatic loop that was trapped inside the crystal. For real in situ Bragg coherent diffraction imaging during nano- 
mechanical tests, it is necessary to record the coherent diffraction patterns by the energy tuning approach, thus avoiding any detrimental vibrations. However, the phase retrieval algorithms used so far are laid-out and optimized for ordinary rocking scans while only one work was reported on the reconstruction of a gold crystal from diffraction data recorded by energy scans [233].

Coherent Diffraction Imaging is developing readily and has great potential for imaging strains and defects inside nanostructures during mechanical testing with a spatial resolution better than 10 $\mathrm{nm}$. Its main drawback presently is the low time resolution (typically $5 \mathrm{~min}$ ) but it is expected to improve considerably (by a factor 100 at least) with the advent of new synchrotron sources as well as free electron lasers that will provide much higher coherent flux.

\section{Conclusions AND OUTLOOK}

The evaluation and understanding of mechanical properties at the nanoscale has been a blooming research field in the last 30 years. Important breakthroughs have been obtained thanks mostly to in-situ observations by scanning or transmission electron microscopy combined with dedicated mechanical testing devices.

The usage of X-ray scattering for studying mechanics at the nanoscale (excepting the case of thin and ultra-thin films) on the contrary is much more recent than electron microscopy investigations, despite the fact $\mathrm{X}$-ray diffraction was used since the very early days as a very sensitive tool to probe lattice distortions. The main reasons for the retardation of $\mathrm{X}$-ray diffraction methods in nanomechanics are related to the need for high photon flux and nanofocusing. The weak interaction of $X$ rays with matter, which is a blessing since it allows for a very straightforward interpretation of scattered intensities via Fourier transform, calls for high enough flux. Thanks to the accomplishments at $3^{\text {rd }}$ generation synchrotron sources, hard X-ray beams are nowadays routinely focused down to the 100-nm scale offering unique opportunities to study the mechanical response of individual nanoobjects without the need of complicated sample preparation, which may alter the specimen microstructure.

To analyse distortions of the crystal lattice in nanostructures under mechanical load various approaches have been established:

- Laue microdiffraction was developed in the 2000s. Since then this technique became mature and is nowadays available on several synchrotron beamlines in the world. It yields lattice orientation, deviatoric strain tensor, and local rotations (e.g. related to dislocation pileups). By measuring the energy of one spot the spherical strain also becomes accessible. Laue microdiffraction is highly compatible with various 
mechanical testing geometries including compression and bending. The technique will benefit from the continuous progress in polishing and shaping of focusing mirrors which should eventually provide beams smaller than $100 \mathrm{~nm}$ in the coming years. Strain and defects mapping is possible via scanning either the sample or the beam itself with a resolution of the order of the beam size. In addition, 3D mapping has been demonstrated but still needs further improvement to be routinely employed for mechanical studies of small scale materials.

- Monochromatic nanodiffraction greatly benefited from the development of focusing optics. Nowadays, $50 \mathrm{~nm}$ hard X-ray beams are routinely available on the long nanofocusing beamlines existing at various facilities worldwide. New experimental strategies using continuous motor scans and on-the-fly image acquisitions allow for fast scanning $X$-ray microscopy where reciprocal space is measured as a function of position in the sample allowing for a detailed evaluation of local strain and defects. The spatial resolution is related to the beam size and the future use of multilayer Laue lenses should bring it down to few $10 \mathrm{~nm}$ in the coming years. The in situ combination with nanoindentation or tensile testing has been successfully demonstrated and the scan of the energy of the incident $\mathrm{X}$-ray beam enabled the measurement of the three-dimensional intensity distribution in reciprocal space during mechanical deformation without the need of moving the sample. Based on the earlier achievements, another X-ray technique has recently been pioneered. Full field X-ray microscopy, which is similar to dark field imaging in TEM allows for imaging a zone of $200 \mu \mathrm{m}$ with a resolution in the 100-nm range [Erreur ! Signet non défini.]. The fact that a complete area is imaged at once without the need of scanning the sample greatly accelerates the experiment facilitating studies on dynamic processes.

- Since the first Bragg Coherent Diffraction Imaging experiments published in 2003 continuous progress has been made thanks to the use of hybrid pixel detectors, improved phase retrieval algorithms, and higher coherent flux. BCDI is certainly the most sensitive method to image lattice displacements ( $1 \mathrm{pm}$ resolution is achievable) with an equivalent spatial resolution smaller than $10 \mathrm{~nm}$. However, the exquisite sensitivity of BCDI to lattice distortions comes with a price: at the present stage it is not possible to phase retrieve coherent diffraction patterns from crystals containing 
several defects or large strain gradients. Combination of BCDI with various in-situ environments has been experimentally demonstrated including nanoindentation and tensile testing.

The further development of faster detectors with larger dynamic range as well as the increased brightness and coherence of next generation synchrotron sources are opening promising avenues for in situ and real time observations of local deformations and dynamics of defects in small-scale materials. Faster detectors and thus shorter exposure times will reduce the demand on the longterm stability and drift reduction of nano-mechanical testing equipment. Consequently, nowadays time consuming techniques such as DAXM or energy dispersive measurements will become compatible with the stability of the testing devices and, hence 3D resolved in situ Laue microdiffraction as well as the in situ measurement of the full strain tensor by energy resolved Laue microdiffraction becomes applicable. While all techniques will profit from new detectors, BCDI is certainly the technique that will benefit the most from the new sources. In particular one may predict a significant progress in time resolution thanks to the increase in coherent flux. Presently, the time needed for recording a sufficiently detailed (i.e. fulfilling the oversampling condition) 3D coherent diffraction pattern is of the order of 5 minutes, which places BCDI far behind TEM, that can image defect dynamics with a typical time resolution of few milliseconds. Of course some pumpprobe experiments have been performed with X-ray scattering with time resolution in the nanosecond time frame [234]. Free electron lasers like the LCLS in Stanford or the X-FEL in Hamburg do provide even higher time resolution down to the picosecond [192] and even femtosecond regime. It should be noted that these type of experiments are typically reserved for reversible processes and cannot be applied to plasticity where pinning of defects and nucleation events make the whole process non reversible. However, given enough flux at free electron lasers the evolution of a system including irreversible processes and defects can be tracked after a single pump, e.g. a shock wave.

\section{ACKNOWLEDGMENT}

The authors gratefully acknowledge D.S Gianola from University of California Santa Barbara (CA, USA), G. Dehm from Max-Planck Institute for Iron Research in Düsseldorf (Germany), and T. Pardoen from Université Catholique in Louvain-la-Neuve (Belgium) for fruitful discussions and proof reading of the manuscript. 


\section{REFERENCES}

[1] S. Suresh, J. Li, Materials science : Deformation of the ultra-strong, Nature 456 (2008) 716

[2] K. Lu K, L. Lu, S. Suresh, Strengthening materials by engineering coherent internal boundaries at the nanoscale, Science 324 (2009) 349

[3] G.F Taylor, A method of drawing metallic filaments and a discussion of their properties and uses, Phys. Rev. 23 (1924) 655

[4] C. Herring, J.K. Galt, Elastic and plastic properties of very small metal specimens, Phys. Rev. 85 (1952) 1060-1061

[5] S.S. Brenner, Tensile strength of whiskers, J. Appl. Phys. 27 (1956) 1484

[6] M.F Doerner, W.D Nix, Stress and deformation processes in thin films on substrates, Critical Reviews in Solid State and Materials Science 14 (1988) 225-268

[7] E. Arzt, G. Dehm, P. Gumbsch, O. Kraft, D. Weiss, Interface controlled plasticity in metals: dispersion hardening and thin film deformation, Prog. Mat. Sci. 46 (2001) 283-307

[8] M.D Thouless, J. Gupta, J.M.E Harper, Stress development and relaxation in copper films during thermal cycling, J. Mat. Res. 8 (1993) 1845-1852

[9] L. Freund, S. Suresh: Thin Film Materials: Stress, Defect formation and surface evolution, Cambridge University Press, 2004

[10] C.V. Thompson, The yield stress of polycrystalline thin films, J. Mat. Res. 8 (1993) 237-238

[11] M.D. Uchic, D.M. Dimiduk, J.N. Florando, W.D. Nix, Sample dimensions influence strength and crystal plasticity, Science 305 (2004) 986-989

[12] M.D. Uchic, D.M. Dimiduk, A methodology to investigate size scale effects in crystalline plasticity using uniaxial compression testing, Mat. Sci. Eng. A 400 (2005) 268

[13] D. Kiener, W. Grosinger, G. Dehm, R. Pippan, A further step towards an understanding of sizedependent crystal plasticity: In situ tension experiments of miniaturized single-crystal copper samples, Acta Mater. 56 (2008) 580-592

[14] R. Maaß, D. Grolimund, S. Van Petegem, M. Willimann, M. Jensen, H. Van Swygenhoven, T. Lehnert, M.A.M. Gijs, C.A. Volkert, E.T. Lilleodden, R. Schwaiger, Defect structure in micropillars using x-ray microdiffraction, Appl. Phys. Lett. 89 (2006) 151905

[15] A.T. Jennings, M.J. Burek, J.R. Greer, Microstructure versus size: Mechnical properties of electroplated single crystalline Cu nanopillars, Phys. Rev. Lett. 104 (2010) 135503

[16] H. Bei, S. Shim, E.P. George, M.K. Miller, E.G. Herbert, G.M. Pharr, Compressive strengths of molybdenum alloy micro-pillars prepared using a new technique, Scripta Mater. 57 (2007) 397400 
[17] H. Bei, S. Shim, M.K. Miller, G.M. Pharr, E.P. George, Effects of ion beam milling on nanomechanical behavior of a Molybdenum-alloy single crystal, Appl. Phys. Lett. 91 (2007) 111915

[18] S. Shim, H. Bei, M.K. Miller, G.M. Pharr, E.P. George, Effects of focused ion beam milling on the compressive behavior of directionally solidified micropillars and the nanoindentation response of an electropolished surface, Acta Mater. 57 (2009) 503-510

[19] G. Richter, K. Hillerich, D.S. Gianola, R. Mönig, O. Kraft, C.A. Volkert, Ultrahigh strength single crystalline nanowhiskers grown by physical vapor deposition, Nano Letters 9 (2009) 3048-3052

[20] J. Michler, K. Wasmer, S. Meier, F. Östlund, K. Leifer, Plastic deformation of gallium arsenide micropillars under uniaxial compression at room temperature, Appl. Phys. Lett. 90 (2007) 043123

[21] F. Östlund, K. Rzepiejewska-Malyska, K. Leifer, L.M. Hale, Y. Tang, R. Ballarini, W.W. Gerberich, J. Michler, Brittle-to-ductile transition in uniaxial compression of silicon pillars at room temperature, Adv. Funct. Mater. 19 (2009) 2439-2444

[22] S. Cuenot, C. Frétigny, S. Demoustier-Champagne, B. Nysten, Surface tension effect on the mechanical properties of nanomaterials measured by atomic force microscopy, Phys. Rev. B 69 (2004) 165410

[23] B. Wen, J.E. Sader, J.J. Boland, Mechanical properties of ZnO nanowires, Phys. Rev. Lett. 101 (2008) 175502

[24] B. Wu, A. Heidelberg, J.J. Boland, Mechanical properties of ultrahigh-strength gold nanowires, Nature Materials 4 (2005) 525-529

[25] R. Agrawal, B. Peng, E.E. Gdoutos, H.D. Espinosa, Elasticity size effects in ZnO nanowires - A combined experimental-computational approach, Nano Lett. 11 (2008) 3668

[26] D. Kiener, W. Grosinger, and G. Dehm, On the importance of sample compliance in uniaxial microtesting, Scripta Mater. 60 (2009) 148

[27] G. Dehm, E. Arzt, In situ transmission electron microscopy study of dislocations in a polycrystalline Cu thin film constrained by a substrate, Appl. Phys. Lett. 77 (2000) 1126

[28] S.H. Oh, M. Legros, D. Kiener, P. Gruber, G. Dehm, In situ TEM straining of single crystal Au films on polyimide: change of deformation mechanisms at the nanoscale, Acta Mat. 55 (2007) 55585571

[29] M. Legros, D.S. Gianola, K. Hemker, In situ TEM observations of fast grain-boundary motion in stressed nanocrystalline aluminium films, Acta Mater. 56 (2008) 3380 
[30] A.M. Minor, E.T. Lilleoden, E.A. Stach, J.W. Morris Jr., In situ transmission electron microscopy study of the nanoindentation behaviour of Al, J. Electronic Materials 31 (2002) 958-964

[31] W. Friedrich, P. Knipping,, M. Laue, Interferenzerscheinungen bei Röntgenstrahlen, Sitzungsberichte der Bayerischen Akademie der Wissenschaften 346 (1913) 971-988

[32] G. Aminoff, X-ray asterism on Laue-photogramms, Geologiska Föreningen i Stockholm Förhandlingar, 41:7 (1919) 534-538

[33] J. Czochralski, Moderne Metallkunde, Berlin verlag von Julius Springer 1924

[34] G.I. Taylor, The mechanism of plastic deformation of crystals, Part I - Theoretical, Proc. Roy. Soc. (London) A145 (1934) 362

[35] M. Polanyi, Z. Physik, 89 (1934) 660

[36] E. Orowan, Z. Physik 89 (1934) 605-613, 614-633, 634-659

[37] H. Lester, R. Aborn, Behavior under stress of the iron crystals, Army Ordnance 6 (1925) 120

[38] R. Glocket and E. Osswald, Unique determination of the principal stresses with X-rays, Z. Tech. Physik 161 (1935) 237

[39] I. Noyan, J. Cohen, Residual Stress: Measurement by Diffraction and Interpretation, Springer, New York (1987)

[40] R. Spolenak, W.L. Brown, N. Tamura, A.A. MacDowell, R.S. Celestre, H.A. Padmore, B. Valek, J.C. Bravman, T. Marieb, H. Fujimoto, B.W. Batterman, J.R Patel, Local plasticity of Al thin films as revealed by X-ray microdiffraction, Phys. Rev. Lett. 90 (2003) 096102

[41] G.A. Chahine, M.-I. Richard, R. A. Homs-Regojo, T.N. Tran-Caliste, D. Carbone, V.L.R. Jacques, R. Grifone, P. Boesecke, J. Katzer, I. Costina, H. Djazouli, T. Shroeder, T.U. Schülli, Imaging of strain and lattice orientation by quick scanning $X$-ray microscopy combined with three-dimensional reciprocal space mapping, J. Appl. Crystallogr. 47 (2014) 762-769

[42] H. Simons, A. King, W. Ludwig, C. Detlefs, W. Pantleon, S. Schmidt, F. Stohr, I. Snigireva, A. Snigirev, H.F. Poulsen, Dark-field X-ray microscopy for multiscale structural characterization, Nature Comm. 6 (2015) 6098

[43] M.A. Pfeifer, G.J. Williams, I.A. Vartanyants, R. Harder, I.K. Robinson, Three-dimensional mapping of a deformation field inside a nanocrystal, Nature 442 (2006) 63-66

[44] A.A. Minkevich, M. Gailhanou, J.-S. Micha, B. Charlet, V. Chamard, O. Thomas, Inversion of the diffraction pattern from an inhomogeneously strained crystal using an iterative algorithm, Phys. Rev B 76 (2007) 104106

[45] D.S. Gianola, C. Eberl, Micro- and nanoscale tensile testing of materials, JOM 61 (2009) 24-35 
[46] G. Dehm, Miniaturized single-crystalline fcc metals deformed in tension: New insights in sizedependent plasticity, Progr. Mater. Sci. 54 (2009) 664-688

[47] H. Van Swygenhoven, S. Van Petegem, In-situ mechanical testing during X-ray diffraction, Materials Characterization 78 (2013) 47-59

[48] R.E. Smallman, R.J. Bishop, Modern Physical Metallurgy and Material Engineeing, ButterworthHeinemann (1999), ISBN: 978-0-7506-4564-5

[49] G.G. Stoney, The tension of metallic films deposited by electrolysis, Proc. Roy. Soc. London A 82 (1909) 172-175

[50] P. Flinn, D. Gardner, W. Nix, Measurement and interpretation of stress in aluminum-based metallization as a function of thermal history, IEEE Trans. Electron Dev. 34 (1987) 689

[51] J. Floro, E. Chason, S. Lee, Real time measurement of epilayer strain using a simplified wafer curvature technique, in Diagnostic techniques for semiconductor materials processing II Book Series, Materials Research Society Symposium Proceedings 406 (1996) 491

[52] M. Kobrinsky, C.V. Thompson, Activation volume for inelastic deformation in polycrystalline Ag films at low temperatures, Acta Mater. 594 (1998) 57

[53] http://esi.oeaw.ac.at/wafer-curvature

[54] X. Li, B. Bushan, A review of nanoindentation continuous stiffness measurement technique and its applications, Materials Characterization 48 (2002) 11-36

[55] D. Tabor, The hardness of metals, Oxford: Oxford Univ. Press (1951)

[56] B. Bhushan, Handbook of micro/nanotribology, 2nd ed. Boca Raton (FL): CRC Press (1999)

[57] D. Tabor, Indentation hardness: fifty years on a personal view, Phil. Mag. A 74 (1996) 12071221

[58] W.C. Oliver, G.M. Pharr, Measurement of hardness and elastic modulus by instrumental indentation: Advances in understanding and refinements to methodology, J. Mater. Res. 19 (2004) 3-20

[59] W.C. Oliver, G.M. Pharr, An improved technique for determining hardness and elastic-modulus using load and displacement sensing indentation experiments, J. Mater. Res. 7 (1992) 15641583

[60] G.M. Pharr, Measurement of mechanical properties by ultra-low load indentation, Mater. Sci. Eng. A 253 (1998) 151-159

[61] H. Hertz, Ueber die Berührug fester elastischer Körper, J. reine angew. Math. 92 (1882) 156

[62] I.N. Sneddon, The relation between load and penetration in the axisymmetric Boussinesq problem for a punch of arbitrary profile, Int. J. Eng. Sci. 3 (1965) 47-57 
[63] M.F. Doerner, W.D. Nix, A method for interpreting the data from depth-sensing indentation instruments, J. Mater. Res. 1 (1986) 601-609

[64] J.B. Pethica, W.C. Oliver, Mechanical properties of nanometer volumes of material: use of the elastic response of small area indentations, In: Bravman JC, Nix WD, Barnett DM, Smith DA, editors. Thin films: stresses and mechanical properties. Mater. Res. Soc. Symp. Proc. vol. 130. Pittsburgh (PA): Materials Research Society (1989) 13-23.

[65] S.A. Syed Asif, J.B. Pethica, Nano scale creep and the role of defects, In: Gerberich WW, Gao H, Sundgren JE, Baker SP, editors. Thin films: stresses and mechanical properties: VI. Mater. Res. Soc. Symp. Proc. 436, Pittsburgh (PA): Materials Research Society (1997) 201-206.

[66] D.M. Dimiduk, M.D. Uchic, T.A. Parthasarathy, Size-affected single-slip behavior of pure nickel microcrystals, Acta Mater. 53 (2005) 4065-4077

[67] http://www.aboutcivil.org/tension-test-tensile-strength-test.html

[68] C.-C. Röhlig, M. Niebelschütz, K. Brueckner, K. Tonisch O. Ambacher, V. Cimalla, Elastic properties of nanowires, Phys. Stat. solidi B 247 (2010) 2557-2570

[69] L. Belliard, T.W. Cornelius, B. Perrin, N. Kacemi, L. Becerra, O. Thomas, M.E. Toimil-Molares, M. Cassinelli, Vibrational response of free standing single copper nanowires through transient reflectivity microscopy, J. Appl. Phys. 114 (2013) 193509

[70] M. Legros, Small scale plasticity - a review, Chapter in "Mechanics of nano-objects", 0. Thomas, A. Ponchet, S. Forest (Ed.), Presses de l'Ecole des Mines (2011)

[71] J. Mayer, L.A. Giannuzzi, T. Kamino, J. Michael, TEM sample preparation and FIB-induced damage, MRS Bulletin 32 (2007) 400 - 407

[72] C.A. Volkert, A.M. Minor, Focused ion beam microscopy and micromachining, MRS Bulletin 32 (2007) 389 - 399

[73] R. Maaß, P.M. Derlet, Micro-plasticity and recent insights from intermittent and small-scale plasticity, Acta Mater. (in press)

[74] M.D. Uchic, P.A. Shade, D.M. Dimiduk, Micro-compression testing of FCC metals: A selected overview of experiments and simulations, JOM Journal of the Minerals, Metals and Materials Society 61 (2009) 36-41

[75] J.R. Greer, W.C. Oliver, W.D. Nix, Size dependence of mechanical properties of gold at the micron scale in the absence of strain gradients, Acta Mater. 53 (2005) 1821-1830. Erratum, Acta Mater. 54 (2006) 1705

[76] C.A. Volkert, E.T Lilleodden, Size effects in the deformation of submicron Au columns, Philos. Mag. 86 (2006) 5567-5579 
[77] C.P. Frick, B.G. Clark, S. Orso, A.S. Schneider, E. Arzt, Size effect on strength and strain hardening of small-scale [111] nickel compression pillars, Mater. Sci. Eng. A 489 (2008) 319-329

[78] D. Kiener, C. Motz, T. Schoberl, M. Jenko, G. Dehm, Determination of mechanical properties of copper at the micron scale, Adv. Eng. Mater. 8 (2006) 1119-1125

[79] E.T. Lilleodden, W.D. Nix, Microstructural length-scale effects in the nanoindentation behavior of thin gold thin films, Acta Mater. 54 (2006) 1583-1593

[80] J.A. El-Awady, Unraveling the physics of size-dependent dislocation-mediated plasticity, Nature Comm. 6 (2015) 5926

[81] A.S. Schneider, D. Kaufmann, B.G. Clark, C.P. Frick, P.A. Gruber, R. Mönig, O. Kraft, E. Arzt, Correlation between critical temperature and strength of small-scale bcc pillars, Phys. Rev. Lett. 103 (2009) 105501

[82] H. Bei, S. Shim, G.M. Pharr, E.P. George, Effects of pre-strain on the compressive stress-strain response of Mo-alloy single-crystal micropillars, Acta Mater. 56 (2008) 4762-4770

[83] J. Zimmermann, S. Van Petegem, H. Bei, D. Grolimund, E.P. George, H. Van Swygenhoven, Effects of focused ion beam milling and pre-straining on the microstructure of directionally solidified molybdenum pillars: A Laue diffraction analysis, Scripta Mater. 62 (2010) 746-749

[84] D. Kiener, C. Motz, M. Rester, M. Jenko, G. Dehm, FIB damage of Cu and possible consequences for miniaturized mechanical tests, Mat. Sci. Eng. A 459 (2007) 262-272

[85] C. Kirchlechner, D. Kiener, C. Motz, S. Labat, N. Vaxelaire, O. Perroud, J.-S. Micha, O. Ulrich, O. Thomas, G. Dehm, J. Keckes, Dislocation storage in single slip-oriented Cu micro-tensile samples: New insights via X-ray diffraction, Phil. Mag. 91 (2010) 1256-1264

[86] S. Cuenot, S. Demoustier-Champagne, B. Nysten, Elastic modulus of polypyrrole nanotubes, Phys. Rev. Lett. 85 (2000) 1690

[87] A. Heidelberg, L.T. Ngo, B. Wu, M.A. Philips, S. Sharma, T.I. Kamins, J.E. Sader, J.J. Boland, A generalized description of the elastic properties of nanowires, Nano Lett. 6 (2006) 1101-1106

[88] E.K. McKarthy, A.T. Bellew, J.E. Sader, J.J. Boland, Poisson's ratio of individual metal nanowires, Nature Comm. 5 (2014) 4336

[89] D. Mordehai, M. Kazakevich, D.J. Srolovitz, E. Rabkin, Nanoindentation size effect in singlecrystal nanoparticles and thin films: A comparative experimental and simulation study, Acta Mater. 59 (2011) 2309-2321 
[90] S. Gravier, M. Coulombier, A. Safi, N. André, A. Boé, J.-P. Raskin, New on-chip nanomechanical testing laboratory - applications to aluminum and polysilicon thin films, J. Microelectromech. S. 18 (2009) 555-569

[91] M. Coulombier, G. Guisbiers, M.-S. Colla, R. Vayrette, J.-P. Raskin, T. Pardoen, On-chip stress relaxation testing method for freestanding thin film materials, Rev. Sci. Instr. 83 (2012) 105004

[92] U. Bhashkar, V. Passi, S. Houri, E. Escobedo-Cousin, S.H. Olsen, T. Pardoen, J.-P. Raskin, On-chip tensile testing of nanoscale silicon free-standing beams, J. Mater. Res. 27 (2012) 571-579

[93] M.-S. Colla, B. Wang, H. Idrissi, D. Schryvers, J.-P. Raskin, T. Pardoen, High strength-ductility of thin nanocrystalline palladium films with nanoscale twins: On-chip testing and grain aggregate model, Acta Mater. 60 (2012) 1795-1806

[94] F.K LeGoues, J.A. Ott, K. Eberl, S.S. Iyer, In situ study of relaxation of SiGe thin films by the modified Frank-Read mechanism, Appl. Phys. Lett. 51 (1992) 173-175

[95] M. Legros, G. Dehm, R.M. Keller-Flaig, E. Arzt, K.J. Hemker, S. Suresh, Dynamic observation of Al thin films plastically strained in a TEM, Mat. Sci. \& Eng. A 309-310 (2001) 463-467

[96] G. Dehm, M. Legros, B. Heiland, In-situ TEM straining experiments of Al films on polyimide using a novel FIB design for specimen preparation, J. Mater. Sci. 41 (2006) 4484-4489

[97] G. Dehm, C. Motz, C. Scheu, H. Clemens, P.H. Mayrhofer, C. Mitterer, Mechanical size-effects in miniaturized and bulk materials, Adv. Eng. Mat. 8 (2006) 1033

[98] S.H. Oh, M. Legros, D. Kiener, G. Dehm, In situ observation of dislocation nucleation and escape in a submicrometre aluminum single crystal, Nature Materials 8 (2009) 95

[99] Z.W. Shan, R.K. Mishra, S.A.S. Asif, O.L. Warren, A.M. Minor, Mechanical annealing and sourcelimited deformation in submicrometre-diameter Ni crystals, Nature Materials 7 (2007) 115-119

[100] D. Kiener, A.M. Minor, Source Truncation and Exhaustion: Insights from quantitative in situ TEM tensile testing, Nano Lett. 11 (2011) 3816-3820

[101] W.M. Mook, C. Niederberger, M. Bechelany, L. Philippe, J. Michler, Compression of freestanding Au nanostructures: from stochastic yield to predictable flow, Nanotechnology 21 (2010) 055701

[102] Y. Zhu, H.D. Espinosa, An electromechanical material testing system for in situ electron microscopy and applications, P. Natl. Acad. Sci. USA 102 (2005) 14503-14508.

[103] J.H. Han, M.T.A. Saif, In situ microtensile stage for electromechanical characterization of nanoscale freestanding films, Rev. Sci. Instrum. 77 (2006) 045102

[104] M.A. Haque, M.T.A. Saif, In-situ tensile testing of nano-scale specimens in SEM and TEM, Experimental Mechanics 42 (2002) 123 
[105] K.F. Murphy, B. Piccionet, M.B. Zanjani, J.R. Lukes, D.S. Gianola, Strain- and defect mediated thermal conductivity in silicon nanowires, Nano Lett. 14 (2014) 3785-3792

[106] P.A. Williams, S.J. Papadakis, M.R. Falvo, A.M. Patel, M. Sinclair, A. Seeger, A. Helser, R.M. Taylor II, S. Washburn, R. Superfine, Controlled placement of an individual carbon nanotube onto a microelectromechanical structure, Appl. Phys. Lett. 80 (2002) 2574-2576

[107] D.S. Gianola, A. Sedlmayr, R. Mönig, C.A. Volkert, R.C. Major, E. Cyrankowski, S.A.S. Asif, O.L. Warren, O. Kraft, In situ nanomechanical testing in focused ion beam and scanning electron microscopes, Rev. Sci. Instr. 82 (2011) 063901

[108] A. Sedlmayr, E. Bitzek, D.S. Ginaola, G. Richter, R. Mönig, O. Kraft, Existence of two twinningmediated plastic deformation modes in Au nanowhiskers, Acta Mater. 60 (2012) 3985-3993

[109] L.Y. Chen, G. Richter, J.P. Sullivan, D.S. Gianola, Lattice anharmonicity in defect-free Pd nanowhiskers, Phys. Rev. Lett. 109 (2012) 125503

[110] L.Y. Chen, M. He, J. Shin, G. Richter, D.S. Gianola, Measuring surface dislocation nucleation in defect-scarce nanostructures, Nature Mater. 15 (2015) 707

[111] J. Sun, L. He, Y.-C. Lo, T. Xu, H. Bi, L. Sun, Z. Zhang, S.X. Mao, J. Li, Liquid-like pseudoelasticity of sub-10-nm crystalline silver particles, Nature Materials 13 (2014) 1007-1012

[112] C. Peng, Y. Zhong, Y. lu, S. Narayanan, T. Zhu, J. Lou, Strain rate dependent mechanical properties in single crystal Ni nanowires, Appl. Phys. Lett. 102 (2013) 083102

[113] A. Minor, J.W. Morris Jr., E.A. Stach, Quantitative in situ nanoindentation in an electron microscope, Appl. Phys. Lett. 79 (2001) 1625

[114] M. Jin, A.M. Minor, E.A. Stach, J.W. Morris Jr., Direct observation of deformation-induced grain growth during nanoindentation of ultrafine-grained Al at room temperature, Acta Mater. 52 (2004) 5381-5387

[115] S. Lee, J. Im, Y. Yoo, E. Bitzek, D. Kiener, G. Richter, B. Kim, S.H. Oh, Reversible cyclic deformation mechanism of gold nanowires by twinning-detwinning transition evidenced from in situ TEM, Nature Comm. 5 (2014) 3033

[116] F. Östlund, P.R. Howie, R. Ghisleni, S. Korte, K. Leifer, W.J. Clegg, J. Michler, Ductile-brittle transition in micropillar compression of GaAs at room temperature, Phil. Mag. 91 (2011) 11901199

[117] R. Ramachandramoorthy, W. Gao, R. Bernal, H. Espinosa, High strain rate tensile testing of silver nanowires: rate-dependent brittle-to-ductile transition, Nano Lett. (2015)

[118] J.M. Wheeler, P. Brodard, J. Michler, Elevated temperature, in situ indentation with calibrated contact temperature, Phil. Mag. 92 (2012) 3128-3141 
[119] J.M. Wheeler, J. Michler, Elevated temperature, nano-mechanical testing in situ in the scanning electron microscope, Rev. Sci. Instr. 84 (2013) 045103

[120] M. Smolka, C. Motz, T. Detzel, W. Robi, T. Griesser, A. Wimmer, G. Dehm, Novel temperature dependent tensile test of freestanding copper thin films, Rev. Sci. Instr. 83 (2012) 064702

[121] B. Warren, X-ray diffraction, Dover (1991)

[122] R. James, The optical principles of the diffraction of $x$-rays, Ox Box Press (1962)

[123] V. Holy, U. Pietsch, T. Baumbach, High resolution $x$-ray scattering from thin films and multilayers, Springer-verlag (1999)

[124] A. Authier, Dynamical theory of X-ray Diffraction, Oxford University Press (2001)

[125] P.P. Ewald, Zur Theorie der Interferenzen der Röntgenstrahlen in Kristallen, Phys. Z. 14 (1913) 465-472.

[126] I.K. Robinson, Crystal truncation rods and surface roughness, Phys. Rev. B 33 (1986) 3830-3836

[127] R. Feidenhans'I, Surface structure determination by X-ray diffraction, Surf. Sci. Rep. 10 (1989) 105-188.

[128] I.K. Robinson, I.A. Vartanyants, G.J. Williams, M.A. Pfeifer, J.A. Pitney, Reconstruction of the shapes of gold nanocrystals using coherent X-ray diffraction, Phys. Rev. Lett. 87 (2001) 195505

[129] A. Giacovazzo, Fundamentals of Crystallography, Oxford University Press (2002)

[130] L. Landau, E. Lifshitz, Theory of Elasticity, Mir (1967)

[131] M. Gailhanou, A. Loubens, J-S. Micha, B. Charlet, A. Minkevich, R. Fortunier, O. Thomas, Strain field in silicon on insulator lines using high resolution X-ray diffraction, Appl. Phys. Lett. 90 (2007) 111914

[132] H. Mimura, S. Handa, T. Kimura, H. Yumoto, D. Yamakawa, H. Yokoyama, S. Matsuyama, K. Inagaki, K. Yamamura, Y. Sano, K. Tamasaku, Y. Nishino, M. Yabashi, T. Ishikawa, K. Yamauchi, Breaking the $10 \mathrm{~nm}$ barrier in hard-X-ray focusing, Nat. Phys. 6 (2009) 122-125.

[133] K.A. Nugent, Coherent methods in the X-ray sciences, Adv. Phys. 59 (2010) 1-99.

[134] A. Diaz, C. Mocuta, J. Stangl, B. Mandl, C. David, J. Vila-Comamala, V. Chamard, T.H. Metzger, G. Bauer, Coherent diffraction imaging of a single epitaxial InAs nanowire using a focused $X$-ray beam, Phys. Rev. B 79 (2009) 125324.

[135] P. Godard, G. Carbone, M. Allain, F. Mastropietro, G. Chen, L. Capello, A. Diaz, T.H. Metzger, J. Stangl, V. Chamard, Three-dimensional high-resolution quantitative microscopy of extended crystals, Nature Comm. 2 (2011) 568.

[136] J.N. Clark, X. Huang, R. Harder, I.K. Robinson, High-resolution three-dimensional partially coherent diffraction imaging, Nature Comm. 3 (2012) 993. 
[137] P. Kirkpatrick, A.V. Baez, Formation of optical images by X-rays, J. Opt. Soc. Am. 38 (1948) 766774.

[138] http://www.spring8.or.jp/en/news_publications/research_highlights/no_50/

[139] A. Snigirev, V. Kohn, I. Snigireva, B. Lengeler, A compound refractive lens for focusing highenergy X-rays, Nature 384 (1996) 49-51.

[140] B. Lengeler, C. Schroer, J. Tümmler, B. Benner, M. Richwin, A. Snigirev, I. Snigireva, M. Drakopoulos, Imaging by parabolic refractive lenses in the hard X-ray range, J. Synchrotron Radiat. 6 (1999) 1153-1167.

[141] I. Snigireva, A. Snigirev, X-ray microanalytical techniques based on synchrotron radiation, J. Environ. Monit. 8 (2006) 33-42

[142] http://www.psi.ch/Imn/diffractive-x-ray-lenses

[143] K. Jefimovs, O. Bunk, F. Pfeiffer, D. Grolimund, J.F. van der Veen, C. David, Fabrication of Fresnel zone plates for hard X-rays, Microelectron. Eng. 84 (2007) 1467-1470.

[144] S.S. Sarkar, H.H. Solak, J. Raabe, C. David, J.F. van der Veen, Fabrication of Fresnel zone plates with $25 \mathrm{~nm}$ zone width using extreme ultraviolet holography, Microelectron. Eng. 87 (2010) 854-858.

[145] J.-S. Chung, G.E. Ice, Automated indexing for texture and strain measurement with broadbandpass x-ray microbeams, J. Appl. Phys. 86 (1999) 5249-5255

[146] B.C. Larson, N. Tamura, J. Chung, G.E. Ice, J.D. Budai, J.Z. Tischler, W. Yang, H. Weiland, W. Lowe, 3-D measurement of deformation microstructure in $\mathrm{Al}(0.2 \%) \mathrm{Mg}$ using submicron resolution white X-ray microbeams, Mat. Res. Soc. Symp. Proc. 590 (2000) 247.

[147] R.I. Barabash, G.E. Ice, N. Tamura, B.C. Larson, G.M. Pharr, K.S. Chung, W. Yang, White microbeam diffraction from distorted crystals, Appl. Phys. Lett. 79 (2001) 749.

[148] B.C. Larson, W. Yang, G.E. Ice, J.D. Budai, J.Z. Tischler, Three-dimensional X-ray structural microscopy with submicrometre resolution, Nature 415 (2002) 887.

[149] A.A. MacDowell, R.S. Celestre, N. Tamura, R. Spolenak, B.C. Valek, W.L. Brown, J.C. Bravman, H.A. Padmore, B.W. Batterman, J.R. Patel, Submicron X-ray diffraction, Nucl. Instr. Meth. A 467-468 (2001) 936.

[150] N. Tamura, A.A. MacDowell, R.S. Celestre, H.A. Padmore, B.C. Valek, J.C. Bravman, R. Spolenak, W.L. Brown, T. Marieb, H. Fujimoto, B.W. Batterman, J. R. Patel High spatial resolution grain orientation and strain mapping in thin films using polychromatic submicron $x$-ray diffraction, Appl. Phys. Lett. 80 (2002) 3724. 
[151] B. C. Valek, J. C. Bravman, N. Tamura, A. A. MacDowell, R. S. Celestre, H. A. Padmore, R. Spolenak, W. L. Brown, B. W. Batterman, J. R. Patel, Electromigration-induced plastic deformation in passivated metal lines, Appl. Phys. Lett. 81 (2002) 4168.

[152] O. Ulrich, X. Biquart, P. Bleuet, O. Geaymond, P. Gergaud, J.-S. Micha, O. Robach, F. Rieutord, A new white beam $x$-ray microdiffraction setup on the BM32 beamline at the European Synchrotron Radiation Facility, Rev. Sci. Instruments 82 (2011) 033908.

[153] Z. Ren, Nanomechanics: combining mechanical testing in situ with focused X-ray diffraction on a synchrotron beamline, PhD thesis, Aix-Marseille Université (2015)

[154] A. Davydok, B.N. Jaya, O. Robach, O. Ulrich, J.-S. Micha, C. Kirchlechner, Analysis of the full stress tensor in a micropillar: Ability of and difficulties arising during synchrotron based $\mu$ Laue diffraction, Materials and Design 108 (2016) 68-75

[155] S. Send, M. von Kozierowski, T. Panzer, S. Gorfman, K. Nurdan, A.H. Walenta, U. Pietsch, W. Leitenberger, R. Hartmann, L. Strüder, Energy-dispersive Laue diffraction by means of a framestore pnCCD, J. Appl. Cryst. 42 (2009) 1139-1146

[156] S. Send, A. Abboud, R. Hartmann, M. Huth, W. Leitenberger, N. Pashniak, J. Schmidt, L. Strüder, U. Pietsch, Characterization of a pnCCD for applications with synchrotron radiation, Nucl. Instr. Meth. A 711 (2013) 132-142

[157] O. Robach, J.-S. Micha, O. Ulrich, P. Gergaud, Full local elastic strain tensor from Laue microdiffraction: simultaneous Laue pattern and spot energy measurement, J. Appl. Cryst. 44 (2011) 688-696

[158] O. Robach, J.-S. Micha, O. Ulrich, O. Geaymond, O. Sicardy, J. Härtwig, F. Rieutord, A tunable multicolour 'rainbow' filter for improved stress and dislocation density field mapping in polycrystals using X-ray Laue microdiffraction, Acta Crystallographica A 69 (2013) 164-170

[159] A. Gassenq, S. Tardif, K. Guilloy, G.O. Dias, N. Pauc, I. Duchemin, D. Rouchon, J.-M. Hartmann, J. Widiez, J. Escalante, Y.-M. Niquet, R. Geiger, T. Zabel, H. Sigg, J. Faist, A. Chelnokov, F. Rieutord, V. Reboud, V. Calvo, Accurate strain measurements in highly strained Ge microbridges, Appl. Phys. Lett. 108 (2016) 241902

[160] S. Tardif, A. Gassenq, K. Guilloy, N. Pauc, G.O. Dias, J ;-M. Hartmann, J. Widiez, T. Zabel, E. Marin, H. Sigg, J. Faist, A. Chelnokov, V. Reboud, V. Calvo, J.-S. Micha, O. Robach, F. Rieutord, Lattice strain and tilt mapping in stressed Ge microstructures using X-ray Laue micro-diffraction and rainbow filtering, J. Appl. Cryst. 49 (2016) 1402-1411 
[161] O. Robach, J.-S. Micha, O. Ulrich, O. Geaymond, O. Sicardy, J. Hartwig, F. Rieutord, A tunable multicolor 'rainbow' filter for improved stress and dislocation density field mapping in polycrystals using X-ray Laue microdiffraction, Acta Cryst. A 69 (2013) 1-7

[162] C. Kirchlechner, J. Keckes, C. Motz, W. Grosinger, P. Imrich, M. Kapp, G. Dehm, Comparison of micron sized tensile and compression tests based on in situ $\mu$ Laue diffraction, Presentation at “Mechanische Charakterisierung in kleinen Dimensionen, Erlangen-Nürnberg University (2010)

[163] N. Tamura, XMAS: A versatile tool for analyzing synchrotron $X$-ray microdiffraction data, Chapter in "Strain and dislocations gradients from diffraction", R. Barabash and G. Ice (Ed.) (Imperial College Press, 2014) pp. 125-155

[164] J.-S. Micha, LaueTools: Open Source Python Packages for X-ray MicroLaue Diffraction Analysis, http://sourceforge.net/p/lauetools/lauetoolswiki/Home (2014).

[165] https://www1.aps.anl.gov/science/scientific-software/lauego

[166] R.I. Barabash, G.E. Ice, Diffraction analysis of defects: State of the art, Chapter in "Strain and dislocation gradients from diffraction", R. Barabash and G. Ice (Ed.), (Imperial College Press, 2014), pp. 1-52

[167] O. Robach, C. Kirchlechner, J.S. Micha, O. Ulrich, X. Biquard, O. Geaymond, O. Castelnau, M. Bornert, J. Petit, O. Sicardy, F. Rieutord, Laue Microdiffraction at the ESRF, Chapter in "Strain and dislocation gradients from diffraction", R. Barabash and G. Ice (Ed.) (Imperial College Press, 2014)

[168] J.P. Hirth, J. Lothe, Theory of dislocations, ISBN-13: 978-0894646171

[169] F. Hofmann, B. Abbey, W. Liu, R. Xu, B.F. Usher, E. Balaur, Y. Liu, X-ray micro-beam characterization of lattice rotations and distortions due to an individual dislocation, Nature Comm. 4 (2013) 2774

[170] J. Petit, M. Bornert, F. Hofmann, O. Robach, J.S. Micha, O. Ulrich, C. Le Bourlot, D. Faurie, A.M. Korsunsky, O. Castelnau, Combining Laue microdiffraction and digital image correlation for improved measurements of the elastic strain field with micrometer spatial resolution, Procedia IUTAM 4 (2012) 133-143

[171] F.G. Zhang, M. Bornert, J. Petit, O. Castelnau, Accuracy of stress measurement by Laue microdiffraction (Laue-DIC method): the influence of image noise, calibration errors and spot number, J. Synchrotron Radiat. 24 (2017) 802-817

[172] F.G. Zhang, O. Castelnau, M. Bornert, J. Petit, J.B. Marijon, E. Plancher, Determination of deviatoric elastic strain and lattice orientation by applying digital image correlation to laue microdiffraction images - the enhanced laue-dic method, J. Appl. Cryst. 48 (2015) 1805-1817 
[173] B.C. Larson, W. Yang, G.E. Ice, J.D. Budai, J.Z. Tischler, Three-dimensional X-ray structural microscopy with submicrometre resolution, Nature 415 (2002) 887-890

[174] O.M. Barabash, M. Sanella, R.I. Barabash, G.E. Ice, J. Tischler, Measuring depth-dependent dislocation densities and elastic strains in an indented Ni-based superalloy, JOM 62 (2010) 29-34

[175] D. Sayre, Some implications of a theorem due to Shannon, Acta Cryst. 5 (1952) 843.

[176] R.W. Gerchberg, W.O. Saxton, A practical algorithm for the determination of the phase from image and diffraction plane pictures, Optik 35 (1972) 237

[177] J.R. Fienup, Reconstruction of an object from the modulus of its Fourier transform, Optics Letters 3 (1978) 27-29

[178] J.R. Fienup, Phase retrieval algorithms: a comparison, Applied Optics 21 (1982) 2758-2769

[179] J.R. Fienup, Reconstruction of a complex-valued object from the modulus of its Fourier transform using a support constraint, J. Opt. Soc. America A 4 (1987) 118

[180] J.R. Fienup, Phase retrieval using boundary conditions, J. Opt. Soc. America A 3 (1986) 284-288

[181] S. Marchesini, H. He, H.N. Chapman, S.P. Hau-Riege, A. Noy, M.R. Howells, U. Weierstall, J.C.H. Spence, X-ray image reconstruction from a diffraction pattern alone, Physical Review B 68 (2003) 140101

[182] K. van Overveld, B. Wyvill, Shrinkwrap: An efficient adaptive algorithm for triangulating an isosurface, The Visual Computer 20 (2004) 362-379

[183] G.J. Williams, M.A. Pfeifer, I.A. Vartanyants, I.K. Robinson, Three-dimensional imaging of microstructure in Au nanocrystals, Phys. Rev. Lett. 90 (2003) 175501

[184] M.C. Newton, S.J. Leake, R. Harder, I.K. Robinson, Three-dimensional imaging of strain in a single ZnO nanorod, Nature Materials 9 (2010) 120-124

[185] V.L.R. Jacques, S. Ravy, D. LeBolloc'h, E. Pinsolle, M. Sauvage-Simkin, F. Livet, Bulk dislocation core dissociation probed by coherent X-rays in silicon, Phys. Rev. Lett. 106 (2011) 065502

[186] V. Favre-Nicolin, F. Mastropietro, J. Eymery, D. Camacho, Y.M. Niquet, B.M. Borg, M.E. Messing, L.-E. Wernersson, R.E. Algra, E.P.A.M. Bakkers, T.H. Metzger, R. Harder, I.K. Robinson, Analysis of strain and stacking faults, in single nanowires using Bragg coherent diffraction imaging, New J. Phys. 12 (2010) 035013

[187] S. Labat, M.-I. Richard, M. Dupraz, M. Gailhanou, G. Beutier, M. Verdier, F. Mastropietro, T.W. Cornelius, T.U. Schülli, J. Eymery, O. Thomas, Inversion domain boundaries in GaN nanowires revealed by coherent Bragg imaging, ACS Nano 9 (2015) 9210-9216 
[188] J.N. Clark, J. Ihli, A.S. Schenk, Y.-Y. Kim, A.N. Kulak, J.M. Campbell, G. Nisbet, F.C. Meldrum, I.K. Robinson, Three-dimensional imaging of dislocation propagation during crystal growth and dissolution, Nature Materials 14 (2015) 780-784

[189] A. Ulvestad, J.N. Clark, R. Harder, I.K. Robinson, O.G. Shpyrko, 3D imaging of twin domain defects in gold nanoparticles, Nano Lett. 15 (2015) 4066-4070

[190] F. Hofmann, E. Tarleton, R.J Harder, N.W. Phillips, P-W. Ma, J.N. Clark, I.K. Robinson, B. Abbey, W. Liu, C.E. Beck, 3D lattice distortions and defect structures in ion-implanted nano-crystals, Sci. Rep. 7 (2017) 45993

[191] A. Ulvestad, J.N. Clark, A. Singer, D. Vine, H.M. Cho, R. Harder, Y.S. Meng, O.G. Shpyrko, In situ strain evolution during a disconnection event in a battery nanoparticle, Phys. Chem. Chem. Phys. 17 (2015) 10551-10555

[192] J.N. Clark. L. Beitra, G. Xiong, A. Higginbotham, D.M. Fritz, H.T. Lemke, D. Zhu, M. Chollet, G.J. Williams, M. Messerschmidt, B. Abbey, R.J. Harder, A.M. Korsunsky, J.S. Wark, I.K. Robinson, Ultrafast three-dimensional imaging of lattice dynamics in individual gold nanocrystals, Science 341 (2013) 56-59

[193] A. Diaz, C. Mocuta, J. Stangl, B. Mandl, C. David, J. Vila-Comamala, V. Chamard, T.H. Metzger, G. Bauer, Coherent diffraction imaging of a single epitaxial InAs nanowire using a focused $x$-ray beam, Phys. Rev. B 79 (2009) 125324

[194] V. Favre-Nicolin, J. Eymery, R. Koester, P. Gentile, Coherent-diffraction imaging of single nanowires of diameter 95 nanometers, Phys. Rev. B 79 (2009) 795401

[195] A. Biermanns, A. Davydok, H. Paetzelt, A. Diaz, V. Gottschalch, T.H. Metzger, U. Pietsch, Individual GaAs nanorods imaged by coherent x-ray diffraction, J. Synchrotron Radiat. 16 (2009) 796-802

[196] V. Chamard, J. Stangl, G. Carbone, A. Diaz, C. Alfonso, C. Mocuta, T.H. Metzger, Threedimensional X-ray Fourier transform holography: The Bragg case, Phys. Rev. Lett. 104 (2010) 165501

[197] V. Chamard, M. Allain, P. Godard, A. Talneau, G. Patriarche, M. Burghammer, Strain in a siliconon-insulator nanostructure revealed by 3D X-ray Bragg ptychography, Sci. Rep. 5 (2015) 9827

[198] Y. Takahashi, A. Suzuki, S. Furutaku, K. Yamauchi, Y. Kohmura, T. Ishikawa, Bragg X-ray ptychography of a silicon crystal: Visualization of the dislocation strain field and the production of a vortex beam, Phys. Rev. B 87 (2013) 121201 
[199] D. Shapiro, P. Thibault, T. Beetz, V. Elser, M. Howells, C. Jacobsen, J. Kirz, E. Lima, H. Miao, A.M. Neiman, D. Sayre, Biological imaging by soft x-ray diffraction microscopy, PNAS 102 (2005) 15343-15346

[200] R. Maaß, S. Van Petegem, H. Van Swygenhoven, P.M. Derlet, C.A. Volkert, D. Grolimund, Timeresolved Laue diffraction of deforming micropillars, Phys. Rev. Lett. 99 (2007) 145505

[201] H. Van Swygenhoven, S. Van Petegem, The use of Laue microdiffraction to study small-scale plasticity, JOM 62 (2010) 36-43

[202] C. Kirchlechner, J. Keckes, J.-S. Micha, G. Dehm, In situ $\mu$ Laue: Instrumental setup for the deformation of micron sized samples, Adv. Eng. Mat. 13 (2011) 837-844

[203] V. Jacques, C. Kirchlechner, R. Ghisleni, L. Thilly, Observation of first plastic events during in-situ compression of InSb micropillars using coherent $x$-rays, ESRF experimental report, http://ftp.esrf.eu/pub/UserReports/50863 A.pdf

[204] J. Gamcová, G. Mohanty, Š. Michalik, J. Wehrs, J. Bednarčik, C. Krywka, J.M. Breguet, J. Michler, H. Franz, Mapping strain fields induced in Zr-based bulk metallic glasses during in-situ nanoindentation by X-ray nanodiffraction, Appl. Phys. Lett. 108 (2016) 031907

[205] A. Zeilinger, J. Todt, C. Krywka, M. Müller, W. Eckert, B. Sartory, M. Meindlhumer, M. Stefenelli, R. Daniel, C. Mitterer, J. Keckes, In-situ observation of cross-sectional microstructural changes and stress distributions in fracturing TiN thin film during nanoindentation, Sci. Rep. 6 (2016) 22670

[206] S. Larcheri, F. Rocca, F. Jandard, D. Pailharey, R. Graziola, A. Kuzmin, J. Purans, X-ray excited optical luminescence detection by scanning near-field optical microscope: A new tool for nanoscience, Rev. Sci. Instr. 79 (2008) 013702

[207] D. Pailharey, Y. Mathey, F. Jandard, S. Larcheri, F. Rocca, A. Kuzmin, R. Kalendarev, J. Purans, G. Dalba, R. Graziola, O. Dhez, Nanoscale x-ray absorption spectroscopy using XEOL-SNOM detection mode, J. Phys. Conf. Ser. 93 (2007) 012038

[208] C.-Y. Chiu, Y.-L. Chan, Y.J. Hsu, D.H. Wei, Collecting photoelectrons with a scanning tunnelling microscope nanotip, Appl. Phys. Lett. 92 (2008) 103101

[209] V. Rose, J.W. Freeland, K.E. Gray, S.K. Streiffer, X-ray excited photoelectron detection using a scanning tunneling microscope, Appl. Phys. Lett. 92 (2008) 193510

[210] T. Scheler, M. Rodrigues, T.W. Cornelius, C. Mocuta, A. Malachias, R. Magalhães-Paniago, F. Comin, J. Chevrier, T.H. Metzger, Probing the elastic properties of individual nanostructures by combining in-situ AFM and micro-X-ray diffraction, Appl. Phys. Lett. 94 (2009) 023109 
[211] B. Gumi-Audenis, F. Carla, M.V. Vitorino, A. Panzarella, L. Porcar, M. Boilot, S. Guerber, P. Bernard, M.S. Rodrigues, F. Sanz, M.I. Giannotti, L. Costa, Custom AFM for X-ray beamlines : in situ biological investigations under physiological conditions, J. Synchrotron Radiat. 22 (2015) 1364-1371

[212] M.S. Rodrigues, O. Dhez, S. Le Denmat, J. Chevrier, R. Felici, F. Comin, Local detection of X-ray spectroscopies with an in-situ atomic force microscope, J. Instrum. 3 (2008) 12004

[213] Z. Ren, F. Mastropietro, S. Langlais, A. Davydok, M.-I. Richard, O. Thomas, M. Dupraz, M. Verdier, G. Beutier, P. Boesecke, T.W. Cornelius, Scanning force microscopy for in situ nanofocused X-ray diffraction studies, J. Synchrotron Radiat. 21 (2014) 1128-1133

[214] C. Leclere, T.W. Cornelius, Z. Ren, A. Davydok, J.-S. Micha, O. Robach, G. Richter, L. Belliard, O. Thomas, In-situ bending of an Au nanowire monitored by micro Laue diffraction, J. Appl. Cryst. 48 (2015) 291-296

[215] T.W. Cornelius, Z. Ren, F. Mastropietro, S. Langlais, A. Davydok, M.-I. Richard, M. Dupraz, M. Verdier, G. Beutier, P. Boesecke, O. Thomas, In situ coupling of atomic force microscopy and sub-micrometer focused X-ray techniques, Mater. Res. Soc. Symp. Proc. 1712 (2014), DOI: 10.1557/opl.2014.909

[216] R. Maaß, S. Van Petegem, C.N. Borca, H. Van Swygenhoven, In situ Laue diffraction of metallic micropillars, Mater. Sci. Eng. A 524 (2009) 40-45

[217] R. Maaß, S. Van Petegem, D. Ma, J. Zimmermann, D. Grolimund, F. Roters, H. Van Swygenhoven, D. Raabe, Smaller is stronger: The effect of strain hardening, Acta Mater. 57 (2009) 5996-6005

[218] C. Marichal, H. Van Swygenhoven, S. Van Petegem, C. Borca, $\{110\}$ slip with $\{112\}$ slip traces in BCC tungsten, Scientific Reports 3 (2013) 2547.

[219] C. Kirchlechner, J. Keckes, C. Motz, W. Grosinger, M.W. Kapp, J.S. Micha, O. Ulrich, G. Dehm, Impact of instrumental constraints and imperfections on the dislocation structure in micronsized Cu compression pillars, Acta Mater. 59 (2011) 5618-5626

[220] C. Kirchlechner, P.J. Imrich, W. Grosinger, M.W. Kapp, J. Keckes, J.S. Micha, O. Ulrich, O. Thomas, S. Labat, C. Motz, G. Dehm, Expected and unexpected plastic behavior at the micron scale: An in situ micro-Laue tensile study, Acta Mater. 60 (2012) 1252-1258.

[221] C. Leclere, T.W. Cornelius, Z. Ren, O. Robach, J.-S. Micha, A. Davydok, O. Ulrich, G. Richter, O. Thomas, KB-scanning of X-ray beam for Laue microdiffraction on accelero-phobic samples: application to in situ mechanically loaded nanowires, J. Synchrotron Radiat. 23 (2016) 13951400 
[222] M. Stefenelli, R. Daniel, W. Ecker, D. Kiener, J. Todt, A. Zeilinger, C. Mitterer, M. Burghammer, J. Keckes, $X$-ray nanodiffraction reveals stress distribution across an indented multilayered $\mathrm{CrN}$-Cr thin film, Acta Mater. 85 (2015) 24-31

[223] M.S. Rodrigues, T.W. Cornelius, T. Scheler, C. Mocuta, A. Malachias, R. Magalhães-Paniago, O. Dhez, F. Comin, T.H. Metzger, J. Chevrier, In situ observation of the elastic deformation of a single epitaxial SiGe crystal by combining atomic force microscopy and micro x-ray diffraction, J. Appl. Phys. 106 (2009) 103525

[224] T.W. Cornelius, D. Carbone, V.L.R. Jacques, T. Schülli, T.H. Metzger, Three-dimensional diffraction mapping by tuning the X-ray energy, J. Synchrotron Radiat. 18 (2011) 413-417

[225] T.W. Cornelius, A. Davydok, V.L.R. Jacques, R. Grifone, T. Schülli, M.-I. Richard, G. Beutier, M. Verdier, T.H. Metzger, U. Pietsch, O. Thomas, In situ 3D reciprocal space mapping during mechanical deformation, J. Synchrotron Radiat. 19 (2012) 688-694

[226] N. Vaxelaire, S. Labat, T.W. Cornelius, C. Kirchlechner, J. Keckes, T. Schulli, O. Thomas, New insights into single-grain mechanical behavior from temperature-dependent 3-D coherent $X$-ray diffraction, Acta Mater. 78 (2014) 46-55

[227] A. Yau, W. Cha, M.W. Kanan, G.B. Stephenson, A. Ulvestad, Bragg coherent diffraction imaging of single-grain defect dynamics in polycrystalline films, Science 356 (2017) 739-742

[228] R. Sutter, Multiscale measurements for materials modeling, Science 356 (2017) 704-705

[229] M. Dupraz, G. Beutier, T.W. Cornelius, G. Parry, Z. Ren, S. Labat, M.-I. Richard, G.A. Chahine, O. Kovalenko, E. Rabkin, M. Verdier, O. Thomas, 3D imaging of a dislocation loop at the onset of plasticity in an indented nanocrystal, Nano Lett. 17 (2017) 6696-6701

[230] M. Dupraz, Diffraction des rayons $X$ cohérents appliqué à la physique du métal, PhD thesis, Université Grenoble Alpes (2015)

[231] J. Shin, T.W. Cornelius, S. Labat, F. Lauraux, M.-I. Richard, G. Richter, N. Blanchard, D.S. Gianola, O. Thomas, In situ Bragg coherent X-ray diffraction during tensile testing of an individual Au nanowire, submitted to Scripta Materialia (under review)

[232] M. Dupraz, G. Beutier, D. Rodney, D. Mordehai, M. Verdier, Signature of dislocations and stacking faults of face-center cubic nanocrystals in coherent X-ray diffraction patterns: $a$ numerical study, J. Appl. Cryst. 48 (2015) 621-644

[233] W. Cha, A. Ulvestad, M. Allain, V. Chamard, R. Harder, S.J. Leake, J. Maser, P.H. Fuoss, S.O. Hruszekewycz, Three-dimensional variable-wavelength X-ray Bragg coherent diffraction imaging, Phys. Rev. Lett. 117 (2016) 225501 
[234] S.O. Mariager, D. Khakhulin, H.T. Lemke, K.S. Kjært, L. Guerin, L. Nuccio, C.B. Sørensen, M.M. Nielsen, R. Feidenhans'l, Direct observation of acoustic oscillations in InAs nanowires, Nano Lett. 10 (2010) 2461-2465 